\title{
Kinetik und Dynamik bei tiefen Temperaturen in Lavaldüsenexpansionen
}

\author{
Dissertation \\ zur Erlangung des Doktorgrades \\ der Mathematisch-Naturwissenschaftlichen Fakultäten \\ der Georg-August-Universität zu Göttingen
}

vorgelegt von

Björn Hansmann

aus Kassel

Göttingen 2007 
D7

Referent: Prof. Dr. J. Troe

Korreferent: Prof. Dr. M. Suhm

Tag der mündlichen Prüfung: 02.05.2007 


\section{Zusammenfassung}

Die temperaturabhängige Messung von Geschwindigkeitskonstanten chemischer Reaktionen und chemischer Dynamik allgemein liefert wichtige Informationen über die zugrundeliegenden molekularen Mechanismen.

Die vorliegende Dissertation untersuchte Reaktionen des Hydroxylradikals $(\mathrm{OH})$ mit den Reaktionspartnern Acetylen, Ethen, Benzol, Alaninethylester, Acetaldehyd und N,N-Dimethylformamid im Temperaturbereich von etwa $60 \mathrm{~K}-300 \mathrm{~K}$. Die Reaktionen von Acetaldehyd und $N, N$-Dimethylformamid wurden zusätzlich in Gegenwart von geringen Mengen Wasserdampf untersucht. Damit handelte es sich um Reaktionspartner steigender molekularer Komplexität. Die Untersuchung der beiden letzten Reaktionen in Gegenwart von Wasser stellte ebenfalls eine Erweiterung zu den Untersuchungen der Vergangenheit dar.

Die Geschwindigkeitskonstanten der Reaktionen des OH-Radikals wiesen mit Ausnahme der Reaktion von Acetylen eine negative Temperaturabhängigkeit auf. Für die Temperaturabhängigkeit der Reaktionen wurden die Höhe von Energiebarrieren in der Reaktionskoordinate relativ zur Energie der isolierten Edukte, die langreichweitigen Wechselwirkungen der Edukte und schwach gebundene Komplexe der Reaktionspartner verantwortlich gemacht.

Die Werte der Geschwindigkeitskonstanten lagen im Bereich von $10^{-12} \mathrm{~cm}^{3} \mathrm{~s}^{-1}$ für Acetylen und im Bereich von $10^{-11} \mathrm{~cm}^{3} \mathrm{~s}^{-1}-10^{-10} \mathrm{~cm}^{3} \mathrm{~s}^{-1}$ für die anderen Reaktionen. Bei Acetylen und Ethen wurden die Geschwindigkeitskonstanten der Schwingungsrelaxation von $\mathrm{OH}(\mathrm{v}=1)$ zur Bestimmung der Hochdruckgeschwindigkeitskonstanten verwendet.

Bei der Reaktion der OH-Radikale mit Acetaldehyd wurde eine Erhöhung der Geschwindigkeitskonstante in Gegenwart von kleinen Mengen Wasserdampf gefunden. Zusammen mit theoretischen Betrachtungen charakteristischer Punkte der Potenti- 
alfläche der Reaktion ließen sich die experimentellen Ergebnisse auf die Existenz von schwach gebundenen Wasserkomplexen der Reaktionspartner zurückführen. Dabei handelte es sich um einen katalytischen Effekt, der von einzelnen Wassermolekülen verursacht wird.

Zur Erzeugung der tiefen Temperaturen bis zu $60 \mathrm{~K}$ diente die Expansion durch verschiedene Lavaldüsen. Bei den kinetischen Untersuchungen diente Stickstoff als Trägergas. Die Machzahl, Temperatur und Dichte im Strahl der Lavaldüse waren konstant. Die Hydroxylradikale wurden durch Photolyse von Wasserstoffperoxid bei den Wellenlängen $193 \mathrm{~nm}$ beziehungsweise $248 \mathrm{~nm}$ erzeugt. Der zeitliche Verlauf der Konzentration von Hydroxylradikalen neben einem Überschuss des Reaktionspartners wurde durch laserinduzierte Fluoreszenz verfolgt.

Neben den Reaktionen des OH-Radikals wurden auch Untersuchungen zum Schwingungsenergietransfer zwischen dem Modellsystem Toluol und den Badgasen Helium, Stickstoff und Kohlendioxid vorgestellt.

Hoch schwingungsangeregte Toluolmoleküle wurden durch die Isomerisierung von Cycloheptatrien nach Photoanregung bei $248 \mathrm{~nm}$ erzeugt. Der zeitliche Verlauf der stoßinduzierten Relaxation von Toluol wurde über die laserinduzierte Fluoreszenz bei verschiedenen Wellenlängen um $267 \mathrm{~nm}$ verfolgt. 


\section{Inhaltsverzeichnis}

1 Einleitung $\quad 1$

2 Theoretische Grundlagen $\quad 15$

2.1 Zwei Modelle der spezifischen Geschwindigkeitskonstante . . . . . . 15

2.2 Temperaturabhängigkeit barriereloser Reaktionen . . . . . . . . . . 18

2.2.1 Rotationsbarrieren bei zentralem Potential . . . . . . . . . 19

2.2.2 Drehimpulserhaltung und Energieerhaltung . . . . . . . . 22

2.2 .3 Anisotropie . . . . . . . . . . . . . 26

2.2.4 Elektronische Beiträge zur Reaktionsgeschwindigkeit . . . . 28

2.2.5 Komplexbildende Reaktionen . . . . . . . . . . . . . 30

2.3 Das Konzept der KCSF für CHT/Toluol . . . . . . . . . . . . . . . 36

3 Experimentelles $\quad 41$

3.1 Isentrope Gasexpansionen . . . . . . . . . . . . . . . . . . 41

3.2 Die Lavaldüse . . . . . . . . . . . . . . . . . . . . . . 43

3.2.1 Berechnung der Wandkontur der Düsen . . . . . . . . . . . . 44

3.2.2 Bestimmung der Bedingungen im Überschallstrahl . . . . . . 51

3.3 Versuchsanordnung der Lavaldüsen-Apparatur . . . . . . . . . . 55

3.3.1 Änderungen bei Messungen von KCSF . . . . . . . . . . . 59

3.4 Datenverarbeitung und zeitliche Steuerung . . . . . . . . . . . . 60

3.5 Verwendete Substanzen . . . . . . . . . . . . . . . . . 63

4 Ergebnisse und Diskussion $\quad 65$

4.1 Schwingungsrelaxation von Toluol . . . . . . . . . . . . 65

4.2 Reaktionen mit ungesättigten Kohlenwasserstoffen . . . . . . . . . . 71 
4.3 Die Reaktion von Alaninethylester mit OH . . . . . . . . . . . . . 79

4.4 Die Reaktion von Acetaldehyd mit $\mathrm{OH}$. . . . . . . . . . . . . . . . 84

4.5 Die Reaktion von $N, N$-Dimethylformamid mit $\mathrm{OH}$. . . . . . . . . 94

5 Ausblick 103

$\begin{array}{ll}\text { Abbildungsverzeichnis } & 105\end{array}$

$\begin{array}{ll}\text { Literaturverzeichnis } & 107\end{array}$ 


\section{Kapitel 1}

\section{Einleitung}

Die Untersuchung der Reaktionen des Hydroxylradikals mit ungesättigten Kohlenwasserstoffen und Carbonylverbindungen bei tiefen Temperaturen trägt zum Verständnis der Chemie der Troposphäre bei. Die Temperaturabhängigkeit von Geschwindigkeitskonstanten über weite Temperaturbereiche gibt Aufschluss über den Reaktionsmechanismus. Bei tiefen Temperaturen wird dabei die Empfindlichkeit der Geschwindigkeitskonstante für Details der Potentialfläche erhöht, auf der die Reaktion stattfindet. So treten beispielsweise schwach gebundene Komplexe auf, die bei größerer thermischer Energie nur wenig Einfluss auf den Reaktionsverlauf besitzen.

Temperaturen unter $200 \mathrm{~K}$ herrschen unter bestimmten Umständen in der Erdatmosphäre in der Tropopause, besonders aber in der Atmosphäre anderer Himmelskörper wie Jupiter, Saturn und Titan. Die Atmosphären dieser Himmelskörper haben eine relativ geringe Dichte, aber dennoch ereignen sich in ihnen genug chemische Reaktionen, um ihre Zusammensetzung zu beeinflussen. Neben Ammoniak und Methan sind dort auch weitere kleine Kohlenwasserstoffe wie Acetylen und Ethen entdeckt worden. Außer bei der Chemie der Atmosphäre anderer Planeten spielt die Untersuchung der Reaktionen bei tiefen Temperaturen für das Verständnis der Zusammensetzung interstellarer Wolken eine wichtige Rolle. In dichten interstellaren Wolken herrschen Temperaturen von 10 - 50 K. Das Adjektiv „dicht“ ist hier allerdings unter astronomischen Bedingungen zu verstehen, dichte interstellare Wolken weisen etwa $10^{6}$ Teilchen pro Kubikzentimeter auf. Ein kleiner Anteil dieser Teilchen sind Moleküle und molekulare Ionen. Zur Entstehung dieser molekularen Teilchen 
tragen nur Reaktionen bei, die unter den vorherrschenden Bedingungen sehr schnell ablaufen.

Die Chemie an diesen Orten wird oftmals durch Modelle simuliert, die Geschwindigkeitskonstanten tiefer Temperaturen als Datengrundlage benötigen [1]. Die Messung der Geschwindigkeitskonstanten bei diesen Temperaturen ist für viele Reaktionen nötig, weil eine Extrapolation von Daten höherer Temperaturen unter Umständen zu großen Abweichungen von den realen Werten führt. In den Modellen kann das falsche Schlussfolgerungen verursachen. Eine Extrapolation kann nur durchgeführt werden, wenn der Reaktionsmechanismus sich nicht ändert und die Temperaturabhängigkeit unverändert bleibt.

Neben der direkten Anwendung in Modellen zur Erklärung der Existenz von Molekülen im interstellaren Raum oder der Zusammensetzung der Atmosphäre von Himmelskörpern gibt es fundamentale Gründe, weshalb die Geschwindigkeit chemischer Reaktionen bei tiefen Temperaturen interessant ist. Die korrekte Beschreibung des Verhaltens bei geringer thermischer Energie und über weite Temperaturbereiche ist für die Modelle chemischer Kinetik eine gute Prüfung. Eine thermische Geschwindigkeitskonstante ist ein stark gemittelter Wert, da die Reaktivität eines Moleküls von seinem quantenmechanischen Zustand abhängt und sehr viele verschiedene Molekülenergien und damit noch mehr Zustände zur thermischen Geschwindigkeitskonstante beitragen. Natürlich sind in der Vergangenheit auch zustandsspezifische Geschwindigkeiten für eine Reihe von Reaktionen experimentell ermittelt worden [2], aber dieser Aufwand ist nicht für viele Reaktionen möglich. Bei niedrigen Temperaturen entstehen schmale thermische Energieverteilungen, die eine Herausforderung für theoretische Modelle darstellen. Der Vergleich mit experimentell ermittelten Daten kann so zur Verbesserung der kinetischen Modelle beitragen.

Ein anderer Aspekt der Prüfung von theoretischen Vorhersagen bezieht sich auf die Existenz von lokalen Maxima in der Reaktionkoordinate oder Eigenschaften der Potentialhyperflächen chemischer Systeme überhaupt. Die Verringerung der thermischen Energie verschiebt die Energiedifferenzen, die sich in der Geschwindigkeitskonstante und ihrer Temperaturabhängigkeit zeigen. Reaktionen werden empfindlich für kleine Barrieren und subtile Eigenschaften der Potentialfläche, auf der 
sie ablaufen. Verhältnismäßig schwache Wechselwirkungen wie Wasserstoffbrücken können bei tiefen Temperaturen für das Reaktionsgeschehen entscheidend sein.

Die Temperaturabhängigkeit vieler Reaktionen lässt sich durch die empirisch gefundene Arrhenius-Gleichung [3] beschreiben. Die Geschwindigkeitskonstante $k(T)$ ist in diesem Fall durch

$$
k(T)=A \cdot \exp \left(\frac{-E_{\mathrm{A}}}{R T}\right)
$$

gegeben. Wobei $A$ eine Konstante mit der Dimension der Geschwindigkeitskonstante darstellt, $R$ steht für die ideale Gaskonstante und $T$ für die Temperatur. Die Größe $E_{\mathrm{A}}$ bezeichnet die Aktivierungsenergie der Reaktion, die mit der Energiebarriere im Potential verknüpft ist.

Reaktionen, die keine ausgeprägte Barriere besitzen, gehorchen der ArrheniusGleichung im Allgemeinen nicht. Zu diesen gehören beispielsweise Reaktionen zwischen Ionen und zwischen Ionen und Neutralteilchen. Hier ist die starke, langreichweitige Coulomb-Wechselwirkung für die Abwesenheit einer absoluten Barriere wichtig und führt zu attraktiven Potentialen. Die zugehörigen Geschwindigkeitskonstanten sind temperaturunabhängig oder weisen eine negative Temperaturabhängigkeit auf.

Das Morse-Potential einer chemischen Bindung weist darauf hin, dass auch Radikal-Radikal Rekombinationen auf barrierefreien Potentialen ablaufen können. Selbst die Reaktionen zwischen Molekülen und Radikalen laufen oft ohne eine Reaktionsbarriere ab.

In Abwesenheit einer ausgeprägten Barriere im Potential ist die Geschwindigkeitskonstante einer Reaktion durch andere Einflüsse bestimmt. Im Abschnitt 2.2 wird darauf näher eingegangen. Wichtige Modelle sind in diesem Zusammenhang statistische Reaktionstheorie und Phasenraumtheorie. Eine weitere Klasse barriereloser Reaktionen, die dort diskutiert wird, sind komplexbildende bimolekulare Reaktionen, die mit Vorteil durch unimolekulare Reaktionskinetik beschrieben werden. Sie zeichnen sich durch die Bildung eines Stoßkomplexes aus, dessen Lebensdauer länger als seine Rotationsperiode ist. Die Temperatur- und Druckabhängigkeit dieser Reaktionen kann ungewöhnliche Änderungen aufweisen. Zum Verständnis dieser 
Reaktionen ist die Betrachtung spezifischer Geschwindigkeitskonstanten unerlässlich.

Die Reaktionen des Hydroxylradikals (OH) mit Acetylen, Ethen, Benzol, Alaninethylester, Acetaldehyd und N,N-Dimethylformamid stellen den Hauptgegenstand dieser Arbeit dar. Alle diese Reaktionen mit Ausnahme des Esters haben eine atmosphärenchemische Bedeutung. Das OH-Radikal ist eines der wichtigsten Oxidantien in der unteren Atmosphäre, deren Chemie besonders vielfältig ist [4].

Das OH-Radikal entsteht dort vornehmlich durch die Photolyse von Ozon. UVStrahlung einer Wellenlänge $\lambda \leq 290 \mathrm{~nm}$ spaltet Ozon in elektronisch angeregte Sauerstoffatome $\mathrm{O}\left({ }^{1} \mathrm{D}\right)$ und elektronisch angeregte Sauerstoffmoleküle $\mathrm{O}_{2}\left({ }^{1} \Delta\right)$. Stöße mit umgebenden Molekülen M stabilisieren beide angeregten Teilchen zu den jeweiligen Grundzuständen, aber ein kleiner Teil der elektronisch angeregten Sauerstoffatome kann vor der Desaktivierung mit Wasser reagieren. Die Sauerstoffatome im elektronischen Grundzustand reagieren schließlich mit $\mathrm{O}_{2}$ zu Ozon. Die von Ozon absorbierte Strahlung wird, besonders in der Stratosphäre, in Wärme umgewandelt. In den folgenden Reaktionsgleichungen werden die oben beschriebenen Prozesse zusammengefasst.

$$
\begin{aligned}
\mathrm{O}_{3}+\mathrm{h} \nu & \rightarrow \mathrm{O}^{*}\left({ }^{1} \mathrm{D}\right)+\mathrm{O}_{2}^{*}\left({ }^{1} \Delta\right) \\
\mathrm{O}^{*}\left({ }^{1} \mathrm{D}\right)+\mathrm{M} & \rightarrow \mathrm{O}\left({ }^{3} \mathrm{P}\right)+\mathrm{M} \\
\mathrm{O}\left({ }^{3} \mathrm{P}\right)+\mathrm{O}_{2} & \rightarrow \mathrm{O}_{3} \\
\mathrm{O}^{*}\left({ }^{1} \mathrm{D}\right)+\mathrm{H}_{2} \mathrm{O} & \rightarrow \mathrm{OH}+\mathrm{OH} .
\end{aligned}
$$

Die Lebensdauer von OH-Radikalen beträgt in der Atmosphäre etwa $10 \mathrm{~s}$, Transportprozesse spielen für die lokale $\mathrm{OH}$ Konzentration also keine Rolle. Die Konzentration beträgt tagsüber etwa $10^{6} \mathrm{~cm}^{-3}$, nachts ist sie deutlich geringer.

Die wichtigsten Reaktionspartner des OH-Radikals sind Kohlenmonoxid und Methan. Das OH-Radikal reagiert zusätzlich mit vielen Spurengasen der Troposphäre und bestimmt deren Atmosphärenchemie. Dazu zählen organische Verbindungen wie Acetylen, Ethen, Benzol und Acetaldehyd, die in die Atmosphäre gelangen. Sie zählen zur Gruppe der flüchtigen organischen Verbindungen, die im atmosphären- 
chemischen Zusammenhang als VOC (volatile organic compounds) bezeichnet werden. Die Reaktion mit dem OH-Radikal stellt den Hauptweg der Eliminierung aus der Atmosphäre dar. Allgemein werden diese Stoffe zunächst durch die Reaktion mit dem OH-Radikal aktiviert und durchlaufen eine Reihe von Abbaureaktionen bis sie entweder zu Kohlendioxid, Wasser und ähnlich stabilen Produkten umgewandelt werden oder durch Wolkenbildung und Regen aus der unteren Atmosphäre ausgewaschen werden.

Die ungesättigten Kohlenwasserstoffe Acetylen, Ethen und Benzol bilden in einer Additionsreaktion zunächst ein Adduktradikal, was dann weitere Oxidationsreaktionen durchläuft. Die Quellen dieser Verbindungen sind sowohl biogen als auch anthropogen. Ethen ist ein Pflanzenhormon und wird von vielen Pflanzen emittiert. Die anderen beiden Kohlenwasserstoffe sind in der Atmosphäre vorwiegend menschlichen Ursprungs.

Acetaldehyd hat in der unteren Atmosphäre sowohl biogene als auch anthropogene Quellen und ist eine der häufigsten Carbonylverbindungen in der Atmosphäre [4]. Als ein wichtiger Vorläufer von Peroxyacetylnitrat (PAN) spielt es eine wichtige Rolle für den Transport von $\mathrm{NO}_{\mathrm{x}}$ in ländliche Gebiete. Die Speicherung von $\mathrm{NO}_{\mathrm{x}}$ in PAN ist eine Eigenschaft, die auch bei der Entstehung von troposphärischem Ozon eine wichtige Rolle spielt. Die Entstehung von PAN aus Acetaldehyd ist hauptsächlich auf die Abstraktion eines Wasserstoffatoms zurückzuführen, der die Addition von $\mathrm{O}_{2}$ und $\mathrm{NO}_{2}$ folgt:

$$
\begin{aligned}
\mathrm{CH}_{3} \mathrm{CHO}+\mathrm{OH} & \longrightarrow \mathrm{CH}_{3} \mathrm{CO}+\mathrm{H}_{2} \mathrm{O} \\
\mathrm{CH}_{3} \mathrm{CO}+\mathrm{O}_{2}+\mathrm{M} & \longrightarrow \mathrm{CH}_{3} \mathrm{C}(\mathrm{O}) \mathrm{O}_{2}+\mathrm{M} \\
\mathrm{CH}_{3} \mathrm{C}(\mathrm{O}) \mathrm{O}_{2}+\mathrm{NO}_{2}+\mathrm{M} & \longrightarrow \mathrm{CH}_{3} \mathrm{C}(\mathrm{O}) \mathrm{O}_{2} \mathrm{NO}_{2}+\mathrm{M}
\end{aligned}
$$

Neben der Photolyse ist die Reaktion mit OH der hauptsächliche Abbaukanal von Acetaldehyd bei Tag. Verschiedene Untersuchungen der primären Produkte der Reaktion zwischen $\mathrm{OH}$ und Acetaldehyd bestätigen die Abstraktion des AldehydWasserstoffs als überwiegenden Reaktionskanal mit einer Primärausbeute von $\mathrm{CH}_{3} \mathrm{CO}$ von mindestens $95 \%$ bei Raumtemperatur [5, 6] und darunter [7]. 
Die Reaktion ist in der vorliegenden Arbeit auch in Gegenwart von 3\% Wasserdampf im Reaktionsgemisch untersucht worden. Seit einiger Zeit wird darüber spekuliert, welchen Einfluss einzelne Wassermoleküle auf die Reaktionen insbesondere von Radikalen in der Gasphase haben können. Wasser kann auf unterschiedliche Weise Gasphasenreaktionen beeinflussen, wobei einige der Effekte auf molekularer Ebene schwer verständlich sind. Relativ gut belegt sind Effekte der Komplexierung von Wasser auf verschiedene photochemische Reaktionen [8]. Die photochemischen Eigenschaften von Ozon [9], Schwefelsäure [10] und Salpetersäure [11]. Die Wasserkomplexe von Radikalen wie $\mathrm{HO}_{2}$ oder $\mathrm{OH}$ könnten eine andere Reaktivität in bimolekularen Reaktionen zeigen als die unkomplexierten Spezies [12]. Die Reaktion von $\mathrm{SO}_{3}$ und Wasser zeigt ein Geschwindigkeitsgesetz zweiter Ordnung in Wasser und der postulierte Mechanismus legt die Beteiligung von zwei Molekülen Wasser bei der Bildung von Schwefelsäure nahe, die als Wasserkomplex gebildet wird $[13,14]$. Die Energietransfereigenschaften von Wasser können ebenso das Reaktionsgeschehen durch die Stabilisierung von Zwischenprodukten beeinflussen. Der Radikalkomplexmechanismus ist mit diesen Transporteigenschaften eng verknüpft und ist eine weitere Form möglichen Wassereinflusses.

Damit sind drei mögliche Arten des Einflusses von Wasserkomplexen auf chemische Reaktionen beschrieben. Die Reaktion zwischen Acetaldehyd und $\mathrm{OH}$ bietet gute Voraussetzungen, Katalyse im engeren Sinne, nämlich die Absenkung von Barrieren zu untersuchen. Die Energietransfereigenschaften von Wasser spielen bei dieser Reaktion, die ohne langlebige Intermediate und unter Beteiligung Wasserstoffbrücken gebundener Komplexe abläuft, keine wichtige Rolle für die Reaktionsgeschwindigkeit. Der geringe Wasseranteil (3\%) trägt zum Energietransfer in gleichem Maße wie das Trägergas Stickstoff $(96 \%)$ bei. Die Reaktion zwischen Acetaldehyd und $\mathrm{OH}$ zeigt bis zu einem Druck von 1000 mbar keine Druckabhängigkeit. Daher ist durch den verwendeten Wasseranteil in den Experimenten keine Veränderung der Geschwindigkeitskonstante über den Energietransfer in Stößen zu erwarten.

Das OH-Radikal hat nicht nur in der Atmosphärenchemie, sondern auch in lebenden Zellen beim Auftreten von oxidativem Stress eine gewisse Bedeutung. Oxidativer Stress von lebenden Zellen wird mit dem Alterungsprozess und Alterskrankheiten wie Arteriosklerose, Alzheimer- und Parkinson-Krankheit in Verbin- 
dung gebracht [15]. Bei einem solch komplexen Prozess sollte jedoch nicht von einer Monokausalität ausgegangen werden. Das OH-Radikal zählt zu einer Gruppe von Radikalen, die als reaktive Sauerstoffspezies (ROS) bezeichnet werden. Das vermehrte Auftreten von ROS wird oxidativer Stress genannt. In den Zellen treten diese Radikale und Oxidationsmittel beispielsweise bei der Herstellung von ATP in den Mitochondrien als Nebenprodukt auf. Bei den Makrophagen des Immunsystems werden sie genauso freigesetzt wie bei Entzündungsreaktionen, wo sie zur Bekämpfung von Krankheitserregern und als Botenstoffe dienen. Die Produktion von ROS in der Atmungskette ist unerwünscht, was durch eine Anzahl spezifischer Enzyme belegt werden kann, die beispielsweise das Superoxid-Anion (Superoxid Dismutase) und Wasserstoffperoxid (Katalase) zu weniger reaktiven Stoffen umwandeln. Neben diesen Enzymen gibt es eine Reihe nichtenzymatischer Antioxidantien (Vitamine A, C, E und Glutathion), die in den Zellen am Abbau reaktiver Sauerstoffspezies beteiligt sind. Die ROS schädigen unspezifisch das Gewebe, mit dem sie in Kontakt stehen, so dass Proteine, Lipide und DNA durch die Reaktion mit ROS verändert werden.

Der Angriff eines Radikals auf ein Protein ist näherungsweise durch den Angriff auf eine seiner Aminosäuren zu beschreiben. In dieser Arbeit ist jedoch nicht die freie Aminosäure untersucht worden, sondern der Ethylester des Alanins. Die freie Aminosäure besitzt einen zu geringen Dampfdruck, um mit der hier verwendeten experimentellen Technik untersucht werden zu können. Theoretische Studien der Reaktion zwischen Alanin und dem OH-Radikal weisen darauf hin, dass die Carboxylgruppe bei der Reaktion eine untergeordnete Rolle spielt [16]. Der Ethylester sollte demnach ein gutes Modellsystem darstellen.

Nachdem die in dieser Arbeit vorgestellten Reaktionen behandelt wurden, wenden sich die folgenden Absätze der experimentellen Methode und ihrer Entwicklung zu.

Die Anforderungen an das Reaktionsmedium bei Durchführung kinetischer Experimente sind eine ausreichend hohe Dichte und konstante Temperatur- und Druckbedingungen über einen hinreichend langen Zeitraum. Die Kondensation der Reaktionspartner an den Wänden eines Reaktionsgefäßes hat lange Zeit die Messung von Geschwindigkeitskonstanten unter $200 \mathrm{~K}$ verhindert. Bis in die achtziger Jahre gab es nur sehr wenige Reaktionen deren Geschwindigkeitskonstanten unter etwa 
200 K experimentell untersucht waren. Eine Ausnahme stellt die Rekombination von Wasserstoffatomen dar, die bei der Siedetemperatur von Stickstoff (77 K) untersucht wurde [17].

Die kinetische Messung im Überschallstrahl einer Lavaldüse, wie sie erstmals von Rowe und seinen Mitarbeitern verwendet wurde, ermöglicht zur Zeit die niedrigsten Temperaturen um chemische Kinetik zu untersuchen. Die experimentelle Methode dieser Arbeit kann Temperaturen zwischen $300 \mathrm{~K}$ und etwa $10 \mathrm{~K}$ erzeugen.

Lavaldüsen finden seit rund 20 Jahren Anwendung in der Untersuchung chemischer Kinetik. Ein Überblick über die Entwicklungen in diesem Feld kann aus Übersichtsartikeln wie Referenzen [18, 19] entnommen werden. Die Arbeitsgruppe von Rowe et al. untersuchte Ionen-Molekülreaktionen in einem $20 \mathrm{~m}$ langen Windkanal in Meudon [20, 21]. Bertrand Rowe prägte den Namen CRESU als Akronym für „cinétique de réaction en encoulement supersonique uniforme" (Reaktionskinetik in uniformer Überschallströmung) für die neue experimentelle Technik.

Mit dem Umzug von Rowes Gruppe zur Université Rennes I wurde 1990 eine Apparatur ausschließlich für die Untersuchung chemischer Kinetik im Überschallstrahl einer Lavaldüse aufgebaut. In dieser Apparatur wurden die Untersuchungen zu Ionen-Molekül-Reaktionen fortgesetzt. Diese Experimente nutzten den Überschallstrahl als Strömungsrohr ohne Wände: die Ionen wurden stets am gleichen Ort in der Expansion erzeugt und in unterschiedlicher Entfernung zum Entstehungsort massenspektrometrisch detektiert, die Reaktionszeit ergab sich bei bekannter Flussgeschwindigkeit aus der Entfernung zwischen Ionisation und Detektion in der Strahlachse. Durch Massenspektrometrie konnten sowohl Edukte als auch Produkte der untersuchten Reaktionen nachgewiesen werden.

Die Arbeitsgruppe von Ian Smith baute im Rahmen einer fortlaufenden Kooperation mit Rowe eine analoge Apparatur in Birmingham auf. Der Schwerpunkt der Forschung in Birmingham lag in der Untersuchung von Reaktionen von neutralen Teilchen, die einem direkten massenspektrometrischen Nachweis nicht zugänglich waren. Es handelte sich hier zumeist um Reaktionen von Radikalen oder Atomen mit Molekülen. Durch gepulste Laserphotolyse eines geeigneten Vorläufers wurde die Reaktion gestartet und die Detektion erfolgte im Allgemeinen durch laserinduzierte Fluoreszenz. Diese beiden Apparaturen arbeiten mit einem kontinuierlichen 
Gasfluss und Düsen, die an der engsten Stelle, dem Düsenhals, einen Durchmesser von minimal einem Zentimeter aufweisen. Der Gasdurchfluss und die erforderlichen beachtlichen Pumpkapazitäten erhöhen die Betriebskosten einer solchen kontinuierlichen Anlage signifikant. In jüngerer Zeit entwickelte die Arbeitsgruppe von M. Smith in den Vereinigten Staaten eine gepulst arbeitende Apparatur, die mit deutlich geringerer Pumpkapazität betrieben werden kann [22, 23]. Von Leone et al. wurde in Berkeley eine ähnliche Apparatur aufgebaut [24, 25], ebenso in Göttingen von Abel et al. die Apparatur, an der die in dieser Arbeit vorgestellten Ergebnisse ermittelt wurden [26, 27, 28]. Im Jahr 2005 erschienen die ersten Ergebnisse einer weiteren Apparatur in Bordeaux [29].

In Tabelle 1.1 sind eine Reihe der bis dato untersuchten Reaktionen mit Angaben zur Herstellung der Radikale bzw. Atome und zur Detektionsmethode aufgeführt. 


\begin{tabular}{|c|c|c|c|c|}
\hline Radikal & Erzeugung & Detektion & Edukte & Referenzen \\
\hline \multicolumn{3}{|c|}{$\mathrm{CN}\left({ }^{2} \Sigma^{+}\right)$a. PLP von NCNO LIF } & $\mathrm{C}_{2} \mathrm{H}_{6}$ & {$[30]$} \\
\hline & @ 583 nm & $(0,0)$-Bande des & $\mathrm{NH}_{3}$ & {$[31]$} \\
\hline & b. PLP von ICN & $\left(\mathrm{B}^{2} \Sigma^{+}-\mathrm{X}^{2} \Sigma^{+}\right)$ & $\mathrm{C}_{2} \mathrm{H}_{2}$ & {$[30]$} \\
\hline & @266 nm & Übergangs & $\mathrm{C}_{2} \mathrm{H}_{4}$ & {$[30]$} \\
\hline & & @388 nm & $\mathrm{CH}_{3} \mathrm{C} \equiv \mathrm{CH}$ & {$[32]$} \\
\hline & & & $\mathrm{CH}_{2}=\mathrm{C}=\mathrm{CH}_{2}$ & {$[32]$} \\
\hline & & & $\mathrm{O}_{2}$ & {$[33,31]$} \\
\hline \multirow[t]{13}{*}{$\mathrm{OH}\left({ }^{2} \Pi\right)$} & $\mathrm{PLP}$ von $\mathrm{H}_{2} \mathrm{O}_{2}$ & LIF & $\mathrm{HBr}$ & {$[23]$} \\
\hline & $@ 266 \mathrm{~nm}$ & $(0,0)$-Bande des & $\mathrm{C}_{2} \mathrm{H}_{2}$ & {$[34]$} \\
\hline & & $\left(\mathrm{A}^{2} \Sigma^{+}-\mathrm{X}^{2} \Pi\right)$ & $\mathrm{C}_{2} \mathrm{H}_{4}$ & {$[35,34]$} \\
\hline & & Übergangs & Propen & {$[35,26]$} \\
\hline & & @308 nm & Buten & {$[35]$} \\
\hline & & & Isopren & {$[26]$} \\
\hline & & & Benzol & {$[28]$} \\
\hline & & & Anthrazen & {$[36]$} \\
\hline & & & $\mathrm{O}\left({ }^{3} \mathrm{P}\right)$ & {$[37]$} \\
\hline & & & NO & {$[38]$} \\
\hline & & & $\mathrm{NO}_{2}$ & {$[18]$} \\
\hline & & & Alaninethyleste & r[28] \\
\hline & & & Acetaldehyd & {$[27]$} \\
\hline \multirow[t]{7}{*}{$\mathrm{C}_{2} \mathrm{H}$} & $\mathrm{PLP}$ von $\mathrm{C}_{2} \mathrm{H}_{2}$ & Chemi- & $\mathrm{C}_{2} \mathrm{H}_{2}$ & {$[39]$} \\
\hline & @193 nm & lumineszenz & $\mathrm{C}_{2} \mathrm{H}_{4}$ & {$[39]$} \\
\hline & & $\mathrm{C}_{2} \mathrm{H}+\mathrm{O}_{2} \rightarrow$ & Propen & {$[39]$} \\
\hline & & $C H\left(A^{2} \Delta\right)$ & $\mathrm{CH}_{3} \mathrm{C} \equiv \mathrm{CH}$ & {$[32]$} \\
\hline & & & $\mathrm{CH}_{2}=\mathrm{C}=\mathrm{CH}_{2}$ & {$[32]$} \\
\hline & & & $\mathrm{O}_{2}$ & {$[39,40]$} \\
\hline & & & Benzol & {$[25]$} \\
\hline \multirow[t]{3}{*}{$\mathrm{CH}$} & Multiphoton & LIF & $\mathrm{CH}_{4}$ & {$[29,41]$} \\
\hline & $\mathrm{PLP}$ von $\mathrm{CHBr}_{3}$ & $(0,0)$-Bande des & $\mathrm{C}_{2} \mathrm{H}_{6}$ & {$[41]$} \\
\hline & @266 nm & $\left(\mathrm{A}^{2} \Delta-\mathrm{X}^{2} \Pi\right)$ & $\mathrm{NH}_{3}$ & {$[42]$} \\
\hline \multicolumn{5}{|c|}{ Fortsetzung auf der nächsten Seite } \\
\hline
\end{tabular}




\begin{tabular}{|c|c|c|c|c|}
\hline Radikal & Erzeugung & Detektion & Edukte & Referenzen \\
\hline \multirow[t]{10}{*}{$\mathrm{CH}$} & & Übergangs & $\mathrm{H}_{2}$ & {$[43]$} \\
\hline & & $@ 430 \mathrm{~nm}$ & $\mathrm{D}_{2}$ & {$[43]$} \\
\hline & & & $\mathrm{N}_{2}$ & {$[44,45]$} \\
\hline & & & $\mathrm{CO}$ & {$[44,45]$} \\
\hline & & & $\mathrm{C}_{2} \mathrm{H}_{2}$ & {$[41]$} \\
\hline & & & $\mathrm{C}_{2} \mathrm{H}_{4}$ & {$[41]$} \\
\hline & & & Buten & {$[41]$} \\
\hline & & & Benzol & {$[18]$} \\
\hline & & & $\mathrm{NO}$ & {$[42]$} \\
\hline & & & $\mathrm{O}_{2}$ & {$[42]$} \\
\hline \multirow[t]{6}{*}{$\mathrm{C}\left({ }^{3} \mathrm{P}_{\mathrm{J}}\right)$} & PLP von $\mathrm{C}_{3} \mathrm{O}_{2}$ & a)Chemilumineszenz & $\mathrm{C}_{2} \mathrm{H}_{2}$ & {$[46][47]$} \\
\hline & 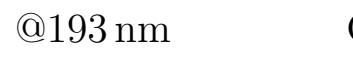 & $\mathrm{C}+\mathrm{NO}_{2} \rightarrow \mathrm{NO}\left(\mathrm{B}^{2} \Pi\right)$ & $\mathrm{C}_{2} \mathrm{H}_{4}$ & {$[46]$} \\
\hline & & b)LIF & $\mathrm{C}_{3} \mathrm{H}_{6}$ & {$[47]$} \\
\hline & & ${ }^{3} \mathrm{P}_{1}-{ }^{3} \mathrm{P}_{0} @ 165.69 \mathrm{~nm}$ & $\mathrm{CH}_{3} \mathrm{C} \equiv \mathrm{CH}$ & {$[46]$} \\
\hline & & & $\mathrm{CH}_{2}=\mathrm{C}=\mathrm{CH}_{2}$ & {$[46]$} \\
\hline & & & $\mathrm{O}_{2}$ & {$[47,48]$} \\
\hline \multirow[t]{3}{*}{$\operatorname{Al}\left({ }^{2} \mathrm{P}_{J}\right)$} & Multiphoton & LIF & $\mathrm{O}_{2}$ & {$[49]$} \\
\hline & PLP von & ${ }^{2} \mathrm{~S}_{1 / 2}-{ }^{2} \mathrm{P}_{\mathrm{J}}$ & & \\
\hline & $\mathrm{Al}\left(\mathrm{CH}_{3}\right)_{3} @ 266 \mathrm{~nm}$ & @394.40 / @396.15 nm & & \\
\hline \multirow[t]{4}{*}{$\mathrm{Si}\left({ }^{3} \mathrm{P}_{\mathrm{J}}\right)$} & Multiphoton- & LIF & $\mathrm{C}_{2} \mathrm{H}_{2}$ & {$[50]$} \\
\hline & PLP von $\mathrm{Si}\left(\mathrm{CH}_{3}\right)_{4}$ & ${ }^{3} \mathrm{P}_{1}-{ }^{3} \mathrm{P}_{0}$ & $\mathrm{C}_{2} \mathrm{H}_{4}$ & {$[50]$} \\
\hline & $@ 266 \mathrm{~nm}$ & $@ 251.43 \mathrm{~nm}$ & NO & {$[51]$} \\
\hline & & & $\mathrm{O}_{2}$ & {$[51]$} \\
\hline \multirow[t]{3}{*}{$\mathrm{B}\left({ }^{2} \mathrm{P}_{\mathrm{J}}\right)$} & PLP von & & $\mathrm{C}_{2} \mathrm{H}_{2}$ & {$[52]$} \\
\hline & $\mathrm{B}\left(\mathrm{CH}_{3} \mathrm{O}\right)_{3}$ & LIF & $\mathrm{C}_{2} \mathrm{H}_{4}$ & {$[53]$} \\
\hline & @266 nm & ${ }^{2} \mathrm{~S}_{1 / 2}-{ }^{2} \mathrm{P}_{\mathrm{J}} @ 249.7 \mathrm{~nm}$ & $\mathrm{O}_{2}$ & {$[54]$} \\
\hline
\end{tabular}

Tabelle 1.1: Diese Tabelle zeigt eine Auswahl an Reaktionen, die mit der CRESU-Technik untersucht wurden. Ionen-Molekülreaktionen sind nicht berücksichtigt. Für Reaktionen von neutralen Spezies wird die Breite der möglichen Reaktionspartner gezeigt. 
Die kinetische Untersuchung chemischer Reaktionen ist nur ein Feld, in dem die uniforme Expansion zur Erweiterung der zugänglichen Temperaturbedingungen beiträgt. Andere Anwendungen finden sich beispielsweise in der IR-Spektroskopie. Der Energietransfer in Stößen [55, 28], die mit Dimerbildung einsetzende Kondensation organischer Moleküle [17], die Bildung von Nanopartikeln [56] bis hin zur Oxidation von einfachen biologischen Bausteinen [28] sind mittels dieser Technik untersucht worden. Der Temperaturbereich wurde durch die Erwärmung [57, 36] oder Abkühlung [58] des Stagnationsvolumens erweitert.

Weitere Anwendungsmöglichkeiten, die für die Apparatur in Göttingen in Frage kommen, werden im Kapitel 5 dargestellt.

Neben der Expansion von Gasen sind prinzipiell andere Methoden denkbar, Moleküle weiter abzukühlen, wie das Abbremsen von Molekularstrahlen mittels StarkEffekts und die Speicherung von Molekülen in elektrostatischen Fallen. Arbeiten, die diese Methode zur Herstellung und zum Speichern ultrakalter Moleküle im Millikelvin-Bereich darstellen, sind von Meijer et al. veröffentlicht worden [59, 60]. Diese Technik könnte die untere Grenze der Temperaturen kinetischer Messungen in Zukunft weiter absenken, sobald die Dichte der gespeicherten Moleküle hoch genug wird um hinreichend viele Stöße während der Speicherungszeit zu ermöglichen.

Eine weitere Anwendung der CRESU Technik im Rahmen dieser Arbeit ist die Untersuchung des Schwingungsenergietransfers in Stößen. In dieser Arbeit wurde der Energietransfer zwischen einer hoch schwingungsangeregten Population von Toluolmolekülen und den Badgasen Helium, Stickstoff und Kohlendioxid untersucht. Die Anwendung des Konzepts auf tiefe Temperaturen stand bei diesem Teil der Arbeit im Vordergrund. Als experimentelle Methode diente die KCSF (kinetically controlled selective fluorescence), die von Frerichs et al. vorgestellt wurde [61]. Die Methode der KCSF für Cycloheptatrien/Toluol, wie sie in dieser Arbeit verwendet wurde, ist im Abschnitt 2.3 genauer beschrieben. Die experimentellen Methoden der zum Vergleich verwendeten Arbeiten werden weiter unten kurz dargestellt.

Der Energietransfer zwischen hoch schwingungsangeregten Molekülen und einem Badgas ist ein Prozess, der bei der Betrachtung von Reaktionskinetik eine wichtige Rolle spielt. Bei Reaktionen, die über eine Barriere laufen, ermöglicht erst die Übertragung von Energie aus dem Badgas die Reaktion. Die Energieverteilung der 
Edukte wird über diesen Prozess (in Konkurrenz zur Reaktion) eingestellt. Bei geringen Drücken ist die Energieübertragung sogar der geschwindigkeitsbestimmende Schritt. Bei Rekombinationsreaktionen hängt die Stabilisierung der chemisch aktivierten Produkte in gleicher Weise vom Energietransfer zwischen dem Badgas und den oft hoch schwingungsangeregten Addukten ab.

Zur Untersuchung des Schwingungsenergietransfers sind verschiedene experimentelle Methoden entwickelt worden. Die UV-Absorptionsmethode (UVA) nutzt die lineare Abhängigkeit der Absorptionskoeffizienten an der Flanke elektronischer Absorptionsbanden von der inneren Energie der Moleküle, um sie zeitaufgelöst zu verfolgen. Thermische Absorptionsspektren aus Stoßwellenexperimenten werden zur Kalibration der UV-Spektren photolytisch angeregter Moleküle verwendet.

Die Infrarot Fluoreszenz (IRF) von schwingungsangeregten Molekülen kann in ähnlicher Weise genutzt werden um die innere Energie angeregter Moleküle zeitlich aufgelöst zu untersuchen. Dabei wird die spontane Emission von IR-Photonen bei Schwingungsübergängen der Moleküle detektiert. Die Intensität der beobachteten Strahlung ist proportional zur Besetzung der Ausgangszustände der betrachteten Übergänge.

Die bisher vorgestellten Methoden erhalten beispielsweise die mittlere pro Stoß übertragene Energie der angeregten Moleküle als Ergebnis. Die von Luther et al. entwickelte Methode der KCSI [62, 63, 64](kinetically controlled selective ionisation) und die verwandte KCSF können neben $\langle\Delta E(E)\rangle$ auch höhere Momente des Energietransfers wie $\left\langle\Delta E^{2}(E)\right\rangle$ bestimmen. Bei einem hinreichen großen Datensatz kann die Übergangswahrscheinlichkeit $P\left(E, E^{\prime}\right)$ ermittelt werden.

Abschließend wird an dieser Stelle die Gliederung der Arbeit erläutert: Zunächst werden im Kapitel 2 theoretische Grundlagen betrachtet, die für die Diskussion und das Verständnis der Ergebnisse wichtig sind. Dabei beginnt das Kapitel mit einer kurzen Beschreibung von Modellen zur Berechnung spezifischer Geschwindigkeitskonstanten und widmet sich dann ausführlicher den Einflüssen auf Geschwindigkeitskonstanten von barrierelosen Reaktionen. Abschließend wird eine einfache Methode zur Analyse der Signale der KCSF von Toluol vorgestellt.

Kapitel 3 beschreibt die experimentelle Technik, die in dieser Arbeit verwendet wurde. Zunächst wird die Entwicklung der CRESU-Methode und die Berechnung 
der Wandkontur von Lavaldüsen dargestellt, darauf folgt die Beschreibung des apparativen Aufbaus. Der letzte Abschnitt beschäftigt sich mit der Auswertung der kinetischen Experimente mit dem OH-Radikal

Kapitel 4 stellt die Ergebnisse der experimentellen Untersuchungen dar und diskutiert sie. Dabei werden theoretische und experimentelle Studien aus der Literatur mit einbezogen. Das Kapitel beginnt mit den Ergebnissen des Schwingungsenergietransfers zwischen Toluol und den Badgasen Helium, Stickstoff und Kohlendioxid. Danach werden die Reaktionen des OH-Radikals mit den ungesättigten Kohlenwasserstoffen Acetylen, Ethen und Benzol betrachtet. Anschließend werden die Ergebnisse der Reaktion von Alaninethylester mit $\mathrm{OH}$ vorgestellt. Zuletzt werden die Reaktionen zwischen $\mathrm{OH}$ mit Acetaldehyd und N,N-Dimethylformamid diskutiert, die zusätzlich in Gegenwart von Wasserdampf untersucht worden sind. 


\section{Kapitel 2}

\section{Theoretische Grundlagen}

In diesem Kapitel werden die theoretischen Grundlagen zur Auswertung und Diskussion der erhaltenen Ergebnisse erarbeitet. Dabei steht die Erarbeitung theoretischer Beschreibungen für barrierelose Reaktionen im Mittelpunkt. Im letzten Abschnitt des Kapitels wird die Analyse der Signale zum Schwingungsenergietransfer dargestellt.

\subsection{Zwei Modelle der spezifischen Geschwindigkeitskonstante}

In diesem Abschnitt werden zwei Modelle zur Berechnung spezifischer Geschwindigkeitskonstanten vorgestellt, wobei auf eine Herleitung verzichtet wird. Die hier vorgestellten Modelle sind zur Berechnung von Molekül-Radikalreaktionen des OHRadikals verwendet worden.

Betrachtet wird eine allgemeine unimolekulare Zerfallsreaktion

$$
\mathrm{AB}^{*} \longrightarrow \mathrm{A}+\mathrm{B}
$$

die über mikroskopische Reversiblität mit der entsprechenden Einfanggeschwindigkeitskonstante verknüpft ist. Nach der RRKM-Theorie [65, 66, 67, 68], benannt nach Rice, Ramsperger, Kassel und Marcus, lässt sich eine unimolekulare Geschwindigkeitskonstante durch folgende Annahmen beschreiben, wie sie in gängigen Lehrbüchern zu unimolekularen Reaktionen $[69,70] \mathrm{zu}$ finden sind: 
- Die Reaktion findet auf einer effektiven Potentialfläche statt, die sich aus dem Potential der Schwingung und Rotation zusammensetzt.

- Der aktivierte Komplex ist eine Oberfläche im Koordinatenraum. Trajektorien, die diese Oberfläche in Richtung Produkte passieren, kehren nicht zurück.

- Die Besetzung aller Mikrozustände einer Energie $E$ ist gleich wahrscheinlich. Die intramolekulare Energieumverteilung (IVR) ist schnell gegenüber der Reaktion.

- Der Prozess des unimolekularen Zerfalls ist eine Translation entlang der Reaktionskoordinate, die von anderen Freiheitsgraden des zerfallenden Moleküls separiert werden kann. Diese Koordinate fehlt dem aktivierten Komplex als Schwingungsfreiheitsgrad.

Die spezifische Geschwindigkeitskonstante wird damit formuliert als

$$
k(E)=\frac{W(E)}{h \rho(E)}
$$

mit $W(E)$, der Anzahl der Zustände des aktivierten Komplexes bis zur Energie $E$, der Planck'schen Konstante $h$ und der Zustandsdichte des zerfallenden Moleküls $\rho(E)$. Die RRKM-Theorie ist gut geeignet zur Beschreibung fester Übergangszustände mit ausgeprägter Energiebarriere. Für einfache Bindungsbruchpotentiale ohne definiertes Energiemaximum im Pfad der minimalen Energie zwischen Edukt und Produkten ist die Konfiguration minimalen Reaktionsflusses nicht mehr durch einen Sattelpunkt in der Potentialfläche gegeben und das Finden dieser Konfiguration erschwert. Für solche Fälle ist die RRKM in ihrer ursprünglichen Form wenig geeignet, allerdings wurden mit der mikrokanonischen VTST (variational transition state theory), PST (phase space theory) und SACM (statistical adiabatic channel model) Methoden zur Berechnung von Reaktionen mit lockerem Übergangszustand entwickelt. Hier wird der Terminus Übergangszustand eigentlich unzutreffend, weil die Fläche des Phasenraums, die den Reaktionsfluss bestimmt, nicht mehr als eine Konfiguration angesehen werden kann, die für alle Zustände gleich ist. Allgemein ist die RRKM-Theorie für Reaktionen mit ausgeprägter Barriere erfolgreich. 
Das statistische Modell adiabatischer Kanäle [71, 72, 73] (SACM) geht für den Grenzfall eines isotropen Potentials in die Phasenraumtheorie über, im Fall eines festen Übergangszustandes nähert sich dieses Modell einer Beschreibung ähnlich der RRKM-Theorie an. Exemplarisch wird das SACM für den Fall einer Bindungsbruchreaktion dargestellt. Für lockere Übergangszustände ist die Erhaltung des Drehimpulses während der Reaktion wichtig, was bei der RRKM-Theorie problematisch ist [74]. Die Berücksichtigung des Drehimpulses wird auch in der Formulierung der spezifischen Geschwindigkeitskonstante ausgedrückt. Die spezifische Geschwindigkeitskonstante ist hier durch

$$
k(E, J)=\frac{W(E, J)}{h \rho(E, J)}
$$

gegeben. $W(E, J)$ ist die Anzahl der Zustände des aktivierten Komplexes im Energiebereich bis $E$ und bei $J$, synonym wird auch die Anzahl der offenen Kanäle verwendet. $\rho(E, J)$ ist die Zustandsdichte des Edukts.

Die Reaktion wird als Bewegung entlang eindimensionaler adiabatischer Kanäle betrachtet. Adiabasie bedeutet in diesem Zusammenhang, dass gute Quantenzahlen der einzelnen Kanäle vom Edukt bis zu den Fragmenten unverändert bleiben, auch wenn entlang der Kanäle verschiedene elektronische Zustände mischen. Die statistische Reaktionstheorie teilt die Freiheitsgrade des zerfallenden Moleküls in einer Bindungsbruchreaktion in zwei Gruppen ein: Eduktschwingungen, die in den Fragmenten als Schwingungen erhalten bleiben, werden erhaltene Oszillatoren genannt. Die Rotationen beziehungsweise Schwingungen des Edukts, die in Eigenrotationen oder relative Rotation der Fragmente übergehen, werden als verschwindende Oszillatoren bezeichnet. Entlang der adiabatischen Kanäle werden die Energieeigenwerte der einzelnen Freiheitsgrade zwischen Edukt- und Fragmentwerten als Funktion der Reaktionskoordinate interpoliert, dabei wird der Interpolationsparameter $\alpha$ verwendet. In dieser Interpolation ist die Flexibilität des Modells begründet, die die Beschreibung sowohl lockerer als auch fest lokalisierter Übergangszustände ermöglicht. Die Kopplung der verschiedenen Freiheitsgrade wird nachträglich nur dazu eingeführt, um die korrekte Behandlung des Drehimpulses zu gewährleisten. Kanäle werden als offen gezählt, wenn ihr Maximum entlang der Reaktionskoordina- 
te den Schwellenwert $E$ nicht erreicht. Als Potential wird im vereinfachten Modell ein Morse-Potential angenommen, das mit den Energien der erhaltenen und verschwindenden Oszillatoren das effektive Potential bildet, allerdings kann für den minimalen Energiepfad ebenso ein geeigneter Schnitt durch eine ab initio Potentialfläche verwendet werden. Nullpunktenergien und Zentrifugalbarrieren gehen in die Berechnung des effektiven Potentials ebenso ein.

Das Verhältnis des Interpolationsparameters zur Kraftkonstante des Morsepotentials $\frac{\alpha}{\beta}$ macht eine qualitative Aussage über den Übergangszustand. Als Standardwert kann $\frac{\alpha}{\beta} \simeq 0.5$ gelten. Höhere Werte weisen auf einen lockeren Übergangszustand hin, kleinere Werte entsprechen einem festen Übergangszustand.

\subsection{Temperaturabhängigkeit barriereloser Reaktionen}

Die Temperatur- und Druckabhängigkeit jeder chemischen Reaktion ist eng verknüpft mit der Wechselwirkung der beteiligten Teilchen auf molekularer Ebene. In Kapitel 1 wurde bereits erörtert, dass die Untersuchung von Reaktionen ohne Energiebarriere bei tiefen Temperaturen besonders lohnend erscheint. In diesem Zusammenhang bezieht sich das Fehlen einer Barriere auf den Weg minimaler Energie ohne Berücksichtigung der Rotationsbarrieren, die auch bei diesen Reaktionen vorhanden sind. Hier werden die theoretischen Grundlagen zur Beschreibung solcher Reaktionen genauer betrachtet. Negative Temperaturabhängigkeit, also ein Wachsen der Geschwindigkeitskonstante bei Erniedrigung der Temperatur, ist bei thermischen Elementarreaktionen, die über eine ausgeprägte Barriere zwischen Edukten und Produkten laufen, nicht zu beobachten. Allerdings ist die Abwesenheit einer Barriere keine hinreichende Bedingung für eine negative Temperaturabhängigkeit. Die folgenden Abschnitte beschäftigen sich mit ausgewählten Einflüssen auf die Geschwindigkeitskonstante, die sie in Abwesenheit einer ausgeprägten Energiebarriere bestimmen. Zuletzt wird die Situation betrachtet, in der nicht ein einzelner Übergangszustand, sondern mehrere, die Geschwindigkeitskonstante beeinflussen. 


\subsubsection{Rotationsbarrieren bei zentralem Potential}

Reaktionen zwischen Ionen und Molekülen aber auch zwischen zwei Radikalen und sogar zwischen gesättigten Molekülen und einem Radikal, wie im Beispiel der Reaktion $\mathrm{CN}+\mathrm{C}_{2} \mathrm{H}_{6}$ [30], können ohne Reaktionsbarriere ablaufen. Der einfachste Fall einer solchen Einfanggeschwindigkeitskonstante, die den Einfang eines polarisierbaren Teilchens und einer Punktladung beschreibt, entspricht der LangevinGeschwindigkeitskonstante. Sie ist temperaturunabhängig und liefert stets den obersten Grenzwert für eine solche Reaktionsgeschwindigkeit, da weder die Eigenbeziehungsweise relative Rotation der Fragmente noch die Anisotropie der Wechselwirkung betrachtet werden müssen. In diese Geschwindigkeitskonstante gehen nur die Polarisierbarkeit, die Ladung des Ions und die reduzierte Masse des Stoßkomplexes ein. Für Reaktionen atomarer Ionen mit apolaren diatomaren Teilchen ist die Übereinstimmung mit experimentellen Daten sehr gut für eine so minimalistische Beschreibung [18].

Bei Einfangreaktionen sind langreichweitige Wechselwirkungen für die Geschwindigkeit wichtig. Sie lassen sich durch ein Potential beschreiben, das in erster Näherung nur vom Abstand der stoßenden Teilchen abhängt. Ein solches zentrales Potential kann bei geladenen Teilchen durch ein thermisch gemitteltes Multipolpotential, bei neutralen Teilchen durch den attraktiven Teil eines Lennard-Jones Potentials oder ein Morse-Potential wiedergegeben werden. Betrachtet wird die Reaktion

$$
\mathrm{A}+\mathrm{B} \longrightarrow \mathrm{M}
$$

dabei ist $\mathrm{M}$ ein polyatomares Molekül, die Fragmente A und B sind dann also Atome, diatomare oder polyatomare Moleküle. Das Bindungspotential steigt zu großen Abständen monoton an, es gibt kein Extremum, mit dem der Übergangszustand identifiziert werden kann. Zunächst wird der Zerfall eines Moleküls über einen lockeren Übergangszustand betrachtet, wie in Abbildung 2.1a dargestellt.

Neben dem abstandsabhängigen Potential $V(r)$ gibt es durch den Drehimpuls $J$ von M noch einen Rotationsbeitrag, so dass das Potential $V_{\text {eff }}^{J}(r)$ den Zerfall für ein rotierendes Molekül M beschreibt. Die Rotation der Fragmente spielt also 


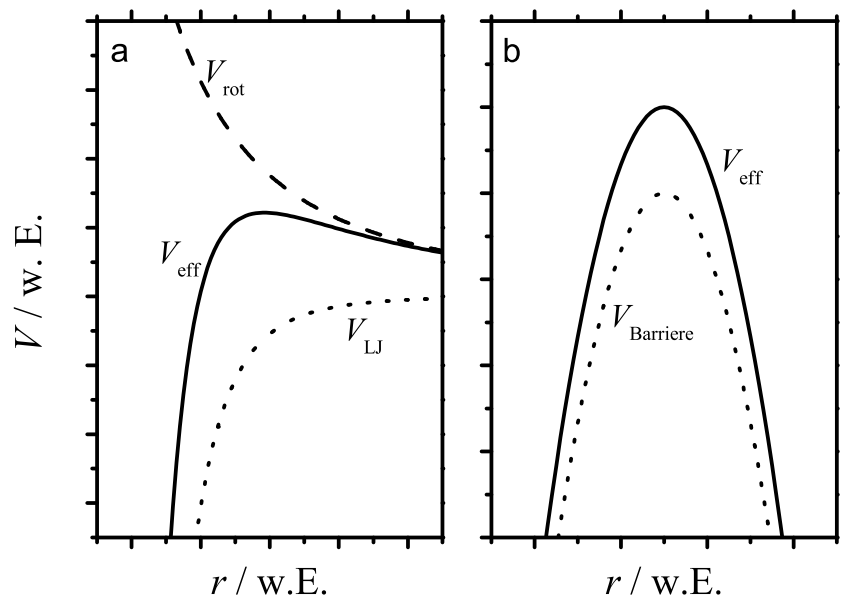

Abbildung 2.1: a zeigt das Potential eines lockeren Übergangszustandes. Dabei bezeichnet die Kurve mit $V_{\text {eff }}$ das effektive Potential, das sich als Summe aus dem Lennard-Jones Teil $V_{\mathrm{LJ}}$ und dem Rotationsteil $V_{\text {Rot }}$ ergibt (vgl. Gl. 2.5). b zeigt das effektive Potential für einen starren Übergangszustand, in erster Näherung wird das rotationsfreie Potential $V_{\text {Barriere gleichmäßig angehoben (vgl. Gl. 2.8) }}$

eine wichtige Rolle, da sie für die Energiemaxima entlang der Reaktionskoordinate verantwortlich ist.

Die Lage des Potentialmaximums wird für zwei Modellpotentiale ermittelt, die einen lockeren und einen festen Übergangszustand qualitativ beschreiben sollen. Das effektive Potential für den lockeren Übergangszustand soll durch

$$
V_{\text {eff }}^{\text {loose }}(r)=-2 E_{0}\left(\frac{r_{\mathrm{e}}}{r}\right)^{6}+J(J+1) B_{\mathrm{e}}\left(\frac{r_{\mathrm{e}}}{r}\right)^{2}
$$

gegeben sein, mit dem Gleichgewichtsabstand $r_{\mathrm{e}}$, der Gleichgewichts-Rotationskonstante $B_{\mathrm{e}}$ und der Bindungsenergie $E_{0}$. Der erste Summand entspricht dem attraktiven Teil eines Lennard-Jones Potentials, der zweite Summand beschreibt das 
Rotationspotential. Die Lage des Potentialmaximums $r_{\max }$ lässt sich durch differenzieren finden:

$$
\left(\frac{r_{e}}{r_{\max }}\right)^{4}=\frac{J(J+1) B_{\mathrm{e}}}{6 E_{0}}
$$

Demnach wird das Maximum der Rotationsbarriere mit steigender Rotationsquantenzahl zu kleineren Abständen verschoben. Die Rotationsbarriere wächst nach

$$
V_{\text {eff }}^{\text {loose }}\left(r_{\max }\right)=\sqrt{\frac{2}{27 E_{0}}}\left[J(J+1) B_{\mathrm{e}}\right]^{3 / 2}
$$

etwa mit $J^{3}$.

Bei einem parabolischen Modellpotential im Reaktionspfad (Abbildung 2.1b), das durch das effektive Potential

$$
V_{\mathrm{eff}}^{\mathrm{rigid}}(r)=-\frac{E_{0}\left(r-r_{0}\right)^{2}}{\left(r_{\mathrm{e}}-r_{0}\right)^{2}}+J(J+1) B_{\mathrm{e}}\left(\frac{r_{\mathrm{e}}}{r}\right)^{2}
$$

wiedergegeben werden kann, zeigt sich ein anderes Verhalten. Hier bezeichnet $E_{0}$ den Scheitelpunkt des parabolischen Potentials an der Stelle $V\left(r_{0}\right)=0$, für $J=0$. Als Extremwert ergibt sich

$$
r_{\max }^{3}\left(r_{0}-r_{\max }\right)=\frac{J(J+1) B_{\mathrm{e}} r_{\mathrm{e}}^{2}\left(r_{\mathrm{e}}-r_{0}\right)^{2}}{E_{0}}
$$

Dabei ist für relevante $J$ die Energiebarriere $E_{0}$ sehr viel größer als die Rotationsbarriere, also $E_{0} \gg J(J+1) B_{\mathrm{e}}$ und damit ist $r_{\max } \simeq r_{0}$. Die Lage der Rotationsbarriere ist für ausgeprägte Energiebarrieren relativ unabhängig von $J$. Die Höhe der Rotationsbarriere ist durch

$$
V_{\mathrm{eff}}^{\mathrm{rigid}}\left(r_{\max }\right) \simeq J(J+1) B_{\mathrm{e}}\left(\frac{r_{\mathrm{e}}}{r_{0}}\right)^{2}
$$

gegeben. 
Sowohl die Lage als auch die Abhängigkeit der Höhe der Rotationsbarrieren von $J$ ist für die beiden Modellpotentiale unterschiedlich. Bei lockerem Übergangszustand wachsen die Rotationsbarrieren für die angegebenen Modellpotentiale stärker mit $J$, allerdings ist die absolute Größe der Rotationsbarrieren bis zu sehr großen $J$ klein verglichen mit der Höhe der Rotationsbarrieren bei festen Übergangszuständen. Qualitativ ist das dadurch erklärbar, dass ein lockerer Übergangszustand bei großen Fragmententfernungen angesiedelt ist wo die absolute Höhe des Rotationspotentials kleiner als bei Entfernungen eines festen Übergangszustandes ist. In den betrachteten Modellpotentialen ist dafür der Faktor bei $B_{\mathrm{e}}$ verantwortlich, der bei beiden Potentialen durch $\frac{r_{\mathrm{e}}}{r_{\max }}$ gegeben ist, siehe Gleichungen 2.5 und 2.8. Für die meisten thermischen Experimente kann gelten, dass die Rotationsbarrieren von kleinen Werten von $J$ bei festen Übergangszuständen größer als bei lockeren sind. Eine weitere Betrachtung der Drehimpulsabhängigkeit verschiedener Übergangszustände wird im Abschnitt 2.2.5 vorgenommen.

Mikrokanonische Geschwindigkeitskonstanten hängen, wie gerade gezeigt, nicht nur von der Energie, sondern auch von der Drehimpulsquantenzahl $J$ ab. Für jede Energie gibt es zusätzlich ein maximales $J$, was die Reaktion gerade noch ermöglicht, was die Zahl der offenen Kanäle reduziert. Die Geschwindigkeitskonstante wird bei der Berücksichtigung der Rotationsbarrieren verringert. Dabei ist nicht nur die Rotationsbarriere, sondern auch die Drehimpulserhaltung zu berücksichtigen, die im nächsten Abschnitt in kurzer Form behandelt wird.

\subsubsection{Drehimpulserhaltung und Energieerhaltung}

Die Rotation von M und den Fragmenten A und B führt zu einem Gesamtdrehimpuls $\bar{J}$, der sich als Vektorsumme aus den Rotationen der Fragmente um Ihren Schwerpunkt - $\overline{j_{1}}$ und $\overline{j_{2}}$ — und der Rotation der Fragmente um den gemeinsamen Schwerpunkt $\bar{L}$ zusammensetzt. Da der Gesamtdrehimpuls eine Erhaltungsgröße ist, wird die Zahl der offenen Kanäle durch diese Randbedingung verringert.

Die Auswirkungen der Drehimpulserhaltung werden in diesem Abschnitt konzeptionell behandelt und bleiben auf allgemeine Betrachtungen beschränkt. Eine ausführlichere Behandlung ist in [69] zu finden. Zur Vereinfachung sei der Drehim- 


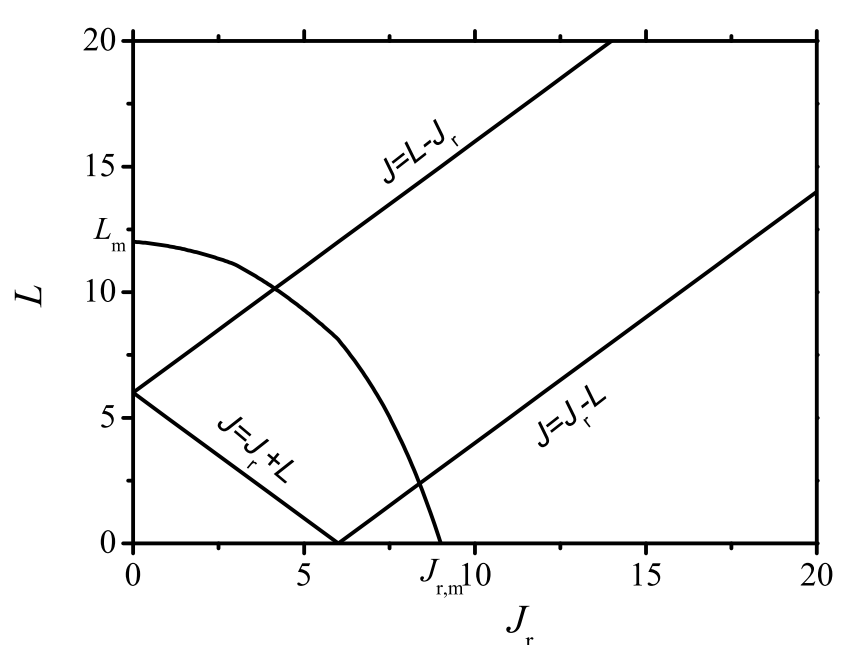

Abbildung 2.2: Eine graphische Darstellung der Drehimpuls- und Energieerhaltung einer Einfangreaktion: Die erlaubten Werte von $L, J_{\mathrm{r}}$ und $J$ ergeben sich als Schnittmenge der Geraden mit der Figur, die durch die Energieerhaltung nach Gleichung 2.18 entsteht

puls der Fragmente zunächst $\overline{j_{1}}+\overline{j_{2}}=\overline{J_{\mathrm{r}}}$. Dann verteilt sich der Gesamtdrehimpuls auf $\overline{J_{\mathrm{r}}}+\bar{L}=\bar{J}$. Graphisch können jetzt die erlaubten Beträge $L$ und $J_{\mathrm{r}}$ für ein festes $J$ dargestellt werden. Eine solche Auftragung findet sich in Abbildung 2.2. Die Achsen bezeichnen die Beträge der beiden Drehimpulsvektoren, die zum Gesamtdrehimpuls koppeln. Die drei Geraden markieren die Grenzen der Werte von $J_{\mathrm{r}}$ und $L$ für einen festen Wert von $J$, der als Achsenabschnitt abgelesen werden kann.

Die möglichen Orbital- und Fragmentdrehimpulse werden zusätzlich durch die Energieerhaltung für die Fragmente eingeschränkt. Dazu muss zunächst die verfügbare Energie betrachtet werden: Die Schwingungsenergie wird nicht betrachtet, da sie sich bei der Assoziation der Fragmente nur adiabatisch ändert. Es sei

$$
y=E_{\mathrm{t}}+E_{\mathrm{r}}
$$


die externe Energie $y$ der Fragmente. Sie setzt sich aus ihrer Rotations- $\left(E_{\mathrm{r}}\right)$ und Translationsenergie $\left(E_{\mathrm{t}}\right)$ zusammen. Ist alle Energie Rotationsenergie, so gilt

$$
y=B_{\mathrm{r}} J_{\mathrm{m}}^{2}
$$

mit dem maximal möglichen Drehimpuls der Fragmente $J_{\mathrm{m}}$ und der effektiven Rotationskonstante $B_{\mathrm{r}}=B_{1} B_{2} /\left(B_{1}+B_{2}\right)$. Allgemein kann die Translationsenergie dann als

$$
E_{\mathrm{t}}=y-E_{\mathrm{r}}=B_{\mathrm{r}} J_{\mathrm{m}}^{2}-B_{\mathrm{r}} J_{\mathrm{r}}^{2}
$$

beschrieben werden. Für ein allgemeines effektives Multipolpotential der Form

$$
V_{\text {eff }}(r)=-\left(\frac{C_{n}}{r^{n}}\right)+\left(\frac{L^{2} \hbar^{2}}{2 \mu r^{2}}\right)
$$

ergibt sich am Potentialmaximum analog Gleichung 2.5

$$
V_{\text {eff }}\left(r_{\max }\right)=\frac{n-2}{2}\left(\frac{L^{2} \hbar^{2}}{n \mu C_{n}^{2 / n}}\right)^{n /(n-2)}
$$

Diese Barrierenhöhe muss durch Translationsenergie überwunden werden. Der Orbitaldrehimpuls $L$ ist also durch

$$
\hbar L=b_{n}\left(B_{\mathrm{r}} J_{\mathrm{m}}^{2}-B_{\mathrm{r}} J_{\mathrm{r}}^{2}\right)^{(n-2) / 2 n}
$$

gegeben. Maximal kann alle verfügbare Energie in der Translation der Fragmente lokalisiert sein, dann ist der maximale Orbitaldrehimpuls $L_{\mathrm{m}}=b_{n} y^{(n-2) / 2 n}$. Die Konstante $b_{n}$ ist definiert als

$$
b_{n}=\frac{1}{\hbar} \sqrt{\frac{2 n \mu}{n-2}}\left[\frac{(n-2) C_{n}}{2}\right]^{1 / n}
$$


mit der reduzierten Masse der Fragmente $\mu$. Damit ist mit der Bedingung der Energieerhaltung zwischen $J_{\mathrm{m}}$ und $L_{\mathrm{m}}$ die Relation

$$
\frac{L}{L_{\mathrm{m}}}=\left[1-\left(\frac{J_{\mathrm{r}}}{J_{\mathrm{m}}}\right)^{2}\right]^{(n-2) / 2 n}
$$

gegeben. Der Wert von $J_{\mathrm{m}}$ kann über einen Koeffizientenvergleich und die Zusammensetzung der verfügbaren Energie bestimmt werden. Diese Kurve begrenzt die in Abbildung 2.2 dargestellten Auswahlmöglichkeiten für $L$ und $J_{\mathrm{r}}$ weiter. Nur Werte innerhalb der Schnittmenge beider Begrenzungen sind erlaubt. Die Grenzen sind anhand der Schnittpunkte beider Bedingungen gegeben.

Zur Berechnung der tatsächlichen Anzahl offener Kanäle, unter Berücksichtigung der Drehimpuls- und Energieerhaltung ist noch die Betrachtung der Entartung der Rotationsniveaus und der Rotationssymmetrie der beteiligten Teilchen nötig. Allgemein ist für die Lösung dieses Problems eine Betrachtung der Grenzfälle für minimalen Gesamtdrehimpuls und maximalen Gesamtdrehimpuls wertvoll. Aus Abbildung 2.2 ist ersichtlich, dass diese Grenzfälle besonders leicht zu lösen sind, weil die Komponenten des Gesamtdrehimpulses nur wenige Werte annehmen können. Ausführlich ist die Lösung für verschiedene Fragmentsymmetrien beispielsweise in Referenz [69] vorgestellt.

Die Geschwindigkeitskonstante, die sich aus den dargestellten Zustandssummen berechnet, ist eine Geschwindigkeitskonstante nach Phasenraumtheorie (PST) [75, $76,77]$. In der vorgestellten Form ist die Erhaltung der Drehimpulse und die Rotationsbarriere berücksichtigt. Ein Vergleich mit experimentellen Daten der spezifischen Geschwindigkeitkonstante zeigt eine überschätzte Energieabhängigkeit der berechneten Geschwindigkeitskonstante, beispielsweise für die Reaktion $\mathrm{NC}+\mathrm{NO} \longrightarrow$ NCNO [78]. Ein Vergleich mit thermischen Hochdruck-Rekombinations-Geschwindigkeitskonstanten $k$ neutraler Teilchen ist für deutlich mehr Systeme möglich. Die Erhöhung von $k$ mit der Temperatur wird in den meisten Fällen überschätzt, einige Reaktionen besitzen eine negative Temperaturabhängigkeit, die nicht durch die PST-Konstante wiedergegeben wird. In einer Form „Konstante $\cdot T^{n}$ “ ausgedrückt liegen die Exponenten der PST-Geschwindigkeitskonstanten kleiner Moleküle zwi- 
schen Null und etwa 0.5. Die experimentellen Werte von $n$ liegen systematisch darunter, um Null herum oder sogar bei Werten unter -0.5. Für Ionen-MolekülReaktionen ist die Verlässlichkeit der PST-Geschwindigkeitskonstanten höher [79]. Es ist naheliegend, dass die Bedingung den Übergangszustand durch die Edukte wiederzugeben aufgrund der länger reichenden intermolekularen Wechselwirkungen besser erfüllt ist. Das Potential dieser Reaktionen wird am Maximum der Rotationsbarriere, die weiter außen liegt als bei neutralen Teilchen, besser durch den attraktiven Term der elektrostatischen Wechselwirkung wiedergegeben. Da die Rotationsbarriere mit sinkendem $J$ nach außen rückt, könnte bei Temperaturen nahe $0 \mathrm{~K}$ diese Beschreibung auch für neutrale Teilchen zutreffend sein.

\subsubsection{Anisotropie}

Bisher wurde das Potential der betrachteten Reaktion als völlig isotrop angenommen. Diese Annahme ist nur bei wenigen Reaktionen gut genug erfüllt um experimentell erhaltene Werte zu reproduzieren. Die Winkelabhängigkeit oder Anisotropie des Einfangpotentials kann mit verschiedenen Ansätzen beschrieben werden. Grundsätzlich wird die PST-Geschwindigkeitskonstante durch Berücksichtigung der Anisotropie reduziert. Zur Berücksichtigung der Winkelabhängigkeit kann beispielsweise die Annäherung der Fragmente von ihrer Eigenrotation separiert werden. Dann ist es möglich den Reaktionsquerschnitt für einen festen Winkel der Fragmente zueinander zu berechnen und über alle möglichen Orientierungen zu mitteln [80]. Diese Näherung führt bei der Verwendung von Multipolpotentialen zu analytischen Ausdrücken für die Geschwindigkeitskonstante, die eine geringe negative Temperaturabhängigkeit aufweisen, oder temperaturunabhängig sind. Die stärkste negative Temperaturabhängigkeit findet sich für Ion-Dipol-Reaktionen $\left(T^{-1 / 2}\right)$.

Die adiabatische Annäherung der Fragmente stellt eine weniger drastische Näherung dar. Dabei wird die Eigenrotation der Fragmente als hinreichend schnell angenommen um sie als gehinderten Rotor zu beschreiben. Bei großen Fragmentabständen rotieren die Fragmente frei, bei sinkendem Abstand nimmt die Hinderung zu. Unabhängige Ansätze von Clary et al. [81] und Troe et al. [82] kommen zu sehr ähnlichen Beschreibungen für diese anisotropen Einfangreaktionen 
wie Ion-Dipol Reaktionen. Die generelle Vorgehensweise zur Lösung ist eine detaillierte Betrachtung mit Hilfe von ab initio Potentialflächen mindestens entlang des Pfades minimaler Energie. Die Ergebnisse aufwändiger Berechnungen von Potentialen und Zustandsdichten dient dann zur Entwicklung und Überprüfung von Näherungen beziehungsweise Korrekturen für PST-Geschwindigkeitskonstanten mit Hilfe von SACM (statistisches Modell adiabatischer Kanäle, siehe Abschnitt 2.1), CT (klassische Trajektorien) oder VMTST (variational mircocanonical transitionstate theory) [83]. Unabhängig vom verwendeten Modell ist die Beschreibung von Ionen-Molekül-Reaktionen sehr erfolgreich, die Vergleiche mit experimentellen Daten haben die Vorhersagekraft dieser Beschreibungen bestätigt [84]. Die Beschreibung der Einfangreaktion neutraler Teilchen ist generell schwieriger: da es sich oft um Radikalreaktionen handelt, sind daran offenschalige Elektronensysteme mit verschiedenen Potentialflächen für die einzelnen elektronischen Zustände beteiligt und die Maxima der offenen Kanäle liegen im Bereich chemischer Wechselwirkungen, die deutlich stärker gerichtet sind als elektrostatische.

Die Rolle der Anisotropie eines Potentials kann in allgemeiner Form nur qualitativ behandelt werden. Da die Anisotropie eine Energieabhängigkeit besitzt, wirkt sie sich direkt auf die Temperaturabhängigkeit einer Reaktion aus. Als Regel kann gelten, dass eine hohe Anisotropie die Geschwindigkeit einer Reaktion senkt. Chemische Wechselwirkungen sind gegenüber elektrostatischen stärker gerichtet, deshalb wirken sie sich stärker aus. Eine geringe negative Temperaturabhängigkeit kann durch die Anisotropie des Potentials erklärt werden. Ohne Kenntnis des Potentials ist die Zurückführung einer negativen Temperaturabhängigkeit auf die Anisotropie eines zugrundeliegenden Potentials nicht belegbar, aber auch nicht auszuschließen. In Referenz [83] und darin zitierten Quellen ist die Herleitung von Korrekturen für PST-Geschwindigkeitskonstanten von Systemen verschiedener Symmetrien aufgeführt. Dabei stellt die PST-Geschwindigkeitskonstante immer einen oberen Grenzwert der Geschwindigkeitskonstante dar. Die Anisotropie der Wechselwirkung der reagierenden Teilchen wird in Form eines Rigiditätsfaktors $f_{\text {rigid }}$ eingeführt. Dieser Faktor wird definiert als

$$
k_{\text {cap }}(T)=f_{\text {rigid }}(T) k_{\text {cap }}^{\mathrm{PST}}(T)
$$


und in mikrokanonischer Form

$$
W(E, J)=f_{\text {rigid }}(E, J) W^{\mathrm{PST}}(E, J)
$$

mit der Geschwindigkeitskonstante nach Phasenraumtheorie $k_{\text {cap }}^{\mathrm{PST}}$, die durch den Rigiditätsfaktor zur Geschwindigkeitskonstante unter Berücksichtigung der Anisotropie $k_{\text {cap }}$ verkleinert wird. Analog dazu bezeichnet $W(E, J)$ die Anzahl der offenen Kanäle bei anisotropem Potential und $W(E, J)^{\mathrm{PST}}$ bei isotropem Potential wie bei Phasenraumtheorie vorausgesetzt. Analytische Ausdrücke für $f_{\text {rigid }}$ können für Modellsysteme durch aufwändige SACM/CT-Rechnungen bestimmt [85] und dann für konkrete Reaktionen verwendet werden.

Auch Neutralreaktionen wurden betrachtet und die Temperaturabhängigkeit im Einklang mit experimentellen Daten wiedergegeben. Die Reaktionen neutraler Spezies, für die eine solche Beschreibung im Einklang mit experimentellen Untersuchungen steht, haben oft eine geringe Temperaturabhängigkeit. Ihre Absolutgeschwindigkeit ist nahe der stoßzahllimitierten Geschwindigkeit [18].

\subsubsection{Elektronische Beiträge zur Reaktionsgeschwindigkeit}

Bei Reaktionen von Radikalen sind durch die Beteiligungen verschiedener elektronischer Zustände weitere Effekte auf die Temperaturabhängigkeit der Geschwindigkeitskonstante möglich. Durch die Spin-Bahn-Kopplung sind bei vielen Radikalen, auch bei $\mathrm{OH}$, verschiedene elektronische Zustände thermisch bis in Temperaturbereiche um $100 \mathrm{~K}$ besetzt. Jeder dieser Zustände führt zu einer eigenen Potentialfläche und damit Geschwindigkeitskonstante. Häufig ist nur der stabilste Zustand reaktiv, die anderen besitzen repulsive Potentiale.

Wenn die Besetzung der Zustände mit der Temperatur verändert wird, ändert sich dadurch auch die Geschwindigkeitskonstante. Das statistische Gewicht der einzelnen Potentialflächen verschiebt sich. Vorrausgesetzt die Population der elektronischen Zustände bleibt durch die Reaktion weitgehend ungestört im Gleichgewicht, ist experimentell keine zustandsspezifische Geschwindigkeitskonstante messbar. Oft ist die Rotation der Kerne mit dem Bahndrehimpuls der Elektronen gekoppelt, so dass die Rotationsrelaxation einen Aufschluss über diese elektronische Relaxation geben 
kann. In der Dissertation von Tim Spangenberg [34] ist die Rotationsrelaxation in der hier verwendeten Lavaldüsenexpansion bestimmt worden. Die Rotationsrelaxation ist unter den in dieser Arbeit vorliegenden Bedingungen um etwa zwei Größenordnungen schneller als die Reaktion. Bei hinreichend schneller elektronischer Relaxation wird die experimentelle Geschwindigkeitskonstante direkt durch das thermische Besetzungsverhältnis der beitragenden elektronischen Zustände beeinflusst. Die Größe des Effekts ist durch die maximale Besetzungsänderung, also den Unterschied zwischen einer Gleichbesetzung aller - und der ausschließlichen Besetzung des reaktiven Zustands gegeben. Nahe der thermischen Energie, die der Energielücke zweier Zustände entspricht, sollte die Geschwindigkeitskonstante einen Abfall mit zunehmender Temperatur zeigen. Beim OH-Radikal treten zwei Komponenten im elektronischen Grundzustand $\mathrm{X}^{2} \Pi_{j}$ auf, $j$ kann die Werte $\frac{3}{2}$ und $\frac{1}{2}$ annehmen. Die Energielücke zwischen der stabileren $j=\frac{3}{2}$ und der $j=\frac{1}{2}$-Komponente beträgt $140 \mathrm{~cm}^{-1}$, was der mittleren thermischen Energie von etwa $100 \mathrm{~K}$ entspricht. Auch die Rotationszustände des OH-Radikals spalten wegen der Kopplung zum elektronischen Drehimpuls zu einem $\Lambda$-Dublett auf. Die Banden des OH-Radikals im UV-Bereich sind in Referenz [86] detailliert beschrieben.

Allerdings gibt es auch experimentelle Daten, die der Annahme widersprechen, dass nur der niedrigste Spin-Bahn-Zustand zur Reaktion beiträgt [18]: Die Gesamtdrehimpulskomponenten des C-Atoms im elektronischen Grundzustand ${ }^{3} \mathrm{P}_{\mathrm{j}}$ weisen eine Erhöhung von $43.4 \mathrm{~cm}^{-3}$ für ${ }^{3} \mathrm{P}_{2}$ und $16.4 \mathrm{~cm}^{-3}$ für ${ }^{3} \mathrm{P}_{1}$ auf. Bei Zimmertemperatur sind diese Zustände gemäß ihrer Entartung besetzt. Bei $13 \mathrm{~K}$, wo Reaktionen von $\mathrm{C}\left({ }^{3} \mathrm{P}_{\mathrm{j}}\right)$ mit der CRESU-Methode (Kinetik in uniformer Gasexpansion: Verwendung von Lavaldüsen zur Untersuchung chemischer Kinetik) untersucht wurden, ist der unterste Zustand beinahe ausschließlich besetzt. Dennoch weisen die Reaktionen in diesem Bereich nur eine sehr schwache Temperaturabhängigkeit auf [46, 47].

Die adiabatische Korrelation zwischen den elektronischen Zuständen der Edukte und Produkte ist bei Radikalreaktionen ebenfalls zu betrachten. Bei Reaktionen von Radikalen mit Singlett-Molekülen entspricht die Multiplizität des Produkts immer der des Radikals. Bei Radikal-Radikal-Reaktionen ergeben sich Potentialflächen verschiedener Multiplizität, wobei die Reaktion nicht auf allen Flächen stattfinden kann. Bei Radikal-Radikal Reaktionen vermindert das Verhältnis der reakti- 
ven zu besetzten Zuständen die Geschwindigkeitskonstante, weil nur ein Teil der Radikal-Radikal-Stöße auf einer reaktiven Potentialfläche stattfindet. Bei der Reaktion $\mathrm{CN}\left({ }^{2} \Sigma^{+}\right)+\mathrm{O}_{2}\left({ }^{3} \Sigma_{\mathrm{g}}^{-}\right)$ist der Anteil beispielsweise ein Drittel [18].

\subsubsection{Komplexbildende Reaktionen: Konkurrenz zweier Reaktionskanäle}

Neben den Radikalreaktionen, die erfolgreich durch Einfanggeschwindigkeitskonstanten unter Berücksichtigung der Anisotropie beschrieben werden können, gibt es eine Reihe von Radikalreaktionen, die eine starke negative Temperaturabhängigkeit besitzen [87, 26, 88]. Die in der Herleitung der Einfanggeschwindigkeitskonstante gemachten Näherungen sind offensichtlich nicht erfüllt: Die Einfanggeschwindigkeit ist nicht mehr der geschwindigkeitsbestimmende Schritt. Für viele dieser Reaktionen ist die Bildung eines stabilen Komplexes während der Reaktion belegt [89]. Komplexbildende bimolekulare Reaktionen haben oft ungewöhnliche Temperatur- und Druckabhängigkeiten [90]. Dabei werden nicht nur negative Temperaturabhängigkeiten gefunden, sondern auch komplexere Verläufe der Geschwindigkeitskonstanten mit Druck und Temperatur.

Wenn die Lebensdauer eines Stoßkomplexes einer bimolekularen Reaktion länger als eine Rotationsperiode dauert, kann sie als komplexbildend bezeichnet werden. Bei hinreichend hohen Drücken können die Komplexe energetisch stabilisiert und in situ detektiert werden. Auch differentielle Reaktionsquerschnitte aus Molekularstrahl-Experimenten können den Nachweis eines langlebigen Intermediats erbringen. Auf theoretischer Seite kann durch die Berechnung der Minima der Potentialfläche ebenfalls ein Hinweis auf Komplexe der Reaktionpartner gegeben werden. Der sicherste Nachweis einer komplexbildenden Reaktion stützt sich natürlich auf experimentelle und theoretische Untersuchungen, die miteinander in Einklang stehen. Allgemein kann eine komplexbildende Reaktion als

$$
\mathrm{A}+\mathrm{B} \longleftrightarrow \mathrm{AB}^{*} \longrightarrow \mathrm{C}+\mathrm{D}
$$




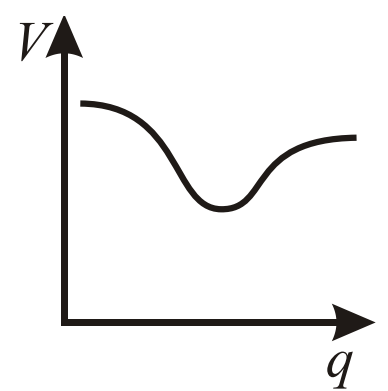

locker / locker

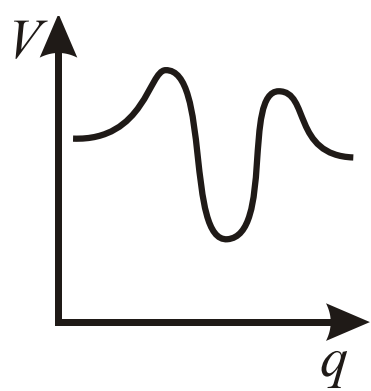

fest / fest

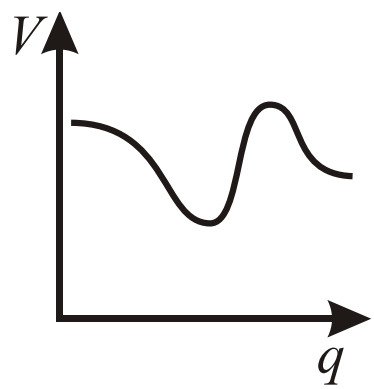

locker / fest

Abbildung 2.3: Die verschiedenen schematisierten Fälle der Eingangs- und Ausgangskanäle einer komplexbildenden bimolekularen Reaktion.

wiedergegeben werden. A und B bilden einen Komplex, der in A und B aber auch in C und D zerfallen kann. Tatsächlich wird die Situation bei einigen Reaktionen noch zusätzlich durch die Konkurrenz zwischen direkter Reaktion und dem komplexbildenden Kanal verkompliziert. Für die Reaktion von $\mathrm{NO}_{2}+\mathrm{O}$ konkurriert die direkte Bildung von $\mathrm{NO}+\mathrm{O}_{2}$ mit der Bildung von $\mathrm{NO}_{3}$ über einen Komplex [91]. Hier sollen nur die Effekte behandelt werden die bei einer komplexbildenden Reaktion auftreten, ohne zusätzliche Kanäle zu anderen Produkten zu behandeln.

In einer komplexbildenden bimolekularen Reaktion ist die Nettogeschwindigkeitskonstante nicht mehr nur von einem, sondern von mehreren Übergangszuständen abhängig, die vom Komplex zu verschiedenen Produkten führen. Dabei können selbst komplizierte Reaktionsmechanismen mit chemischer Aktivierung wie beispielsweise die Reaktion $\mathrm{C}_{2} \mathrm{H}_{2}+\mathrm{OH}$ [92] sich formal in die Situation zerlegen lassen, in der ein Komplex mehrere unimolekulare Zerfallskanäle besitzt, die in direkter Konkurrenz zueinander stehen. Die genauere Behandlung beschränkt sich an dieser Stelle auf die Situation zweier Übergangszustände. Sowohl Eingangs, als auch Ausgangskanal können jeweils über einen lockeren oder festen Übergangszustand laufen, wodurch sich schematisch drei Fälle ergeben, die in Abbildung 2.3 darge- 
stellt sind: locker/locker, fest/fest und locker/fest. Dabei sollen die beiden Zerfälle durch die Reaktionsgleichungen

$$
\mathrm{AB}^{*} \stackrel{\mathrm{k}_{\mathrm{diss}, \mathrm{r}}}{\longrightarrow} \mathrm{A}+\mathrm{B}
$$

und

$$
\mathrm{AB}^{*} \stackrel{\mathrm{k}_{\mathrm{diss}, \mathrm{v}}}{\longrightarrow} \mathrm{C}+\mathrm{D}
$$

bezeichnet werden. Als Geschwindigkeitsgesetz ergibt sich formal

$$
-\frac{\mathrm{d}[\mathrm{A}]}{\mathrm{d} t}=\frac{\mathrm{d}[\mathrm{C}]}{\mathrm{d} t}=k_{\text {cap }}\left[\frac{k_{\mathrm{diss}, \mathrm{v}}}{k_{\mathrm{diss}, \mathrm{v}}+k_{\mathrm{diss}, \mathrm{r}}}\right][\mathrm{A}][\mathrm{B}]
$$

mit der Komplexbildungskonstante $k_{\text {cap }}$, die eine Einfanggeschwindigkeitskonstante darstellt, wie sie in den vorangegangenen Abschnitten erarbeitet wurde. Die Betrachtung wird zunächst unter Vernachlässigung des Energietransfers durchgeführt. Die beiden Zerfallskonstanten des Komplexes werden mit $k_{\text {diss,v }}$ für die Dissoziation des Komplexes vorwärts zu den Produkten und rückwärts $k_{\text {diss,r }}$ zu den Edukten bezeichnet. Die Rückreaktion ist über mikroskopische Reversibilität mit der Einfangkonstante gekoppelt. Die Energie- und Drehimpulsabhängigkeit jeder dieser Konstanten muss in einem solchen Fall berücksichtigt werden. Jede einzelne Konstante wird durch eine spezifische Geschwindigkeitskonstante

$$
k(E, J)=\frac{W(E, J)}{h \rho(E, J)}
$$

wiedergegeben (vgl. Abschnitt 2.1). Da sich die Zustandsdichten der Geschwindigkeitskonstanten im Klammerausdruck von Gleichung 2.24 auf den Komplex beziehen, kürzen sie sich aus dem Ausdruck heraus und für die Nettogeschwindigkeitskonstante $k$ ergibt sich im hier betrachteten Niederdruckfall

$$
k=\frac{k_{\mathrm{B}}}{h}\left(\frac{Q_{\mathrm{AB}}}{Q_{\mathrm{A}} Q_{\mathrm{B}}}\right)_{\mathrm{el}, \text { trans }}\left(\frac{Q_{\mathrm{AB}}^{*} Y}{Q_{\mathrm{A}} Q_{\mathrm{B}}}\right)_{\mathrm{vib}, \operatorname{rot}}
$$


mit den elektronischen, translatorischen und rovibronischen Zustandsdichten von $\mathrm{A}, \mathrm{B}$ und $\mathrm{AB}^{*}$ und für Verzweigungsfaktor $Y$ der mit

$$
Q_{\mathrm{AB}}^{*} Y=\sum_{J=0}^{\infty}(2 J+1) \int_{0}^{\infty} \frac{\mathrm{d} E}{k_{\mathrm{B}} T} \exp \left(-\frac{E}{k_{\mathrm{B}} T}\right) W_{\mathrm{a}}(E, J) \cdot\left[\frac{W_{\mathrm{b}}(E, J)}{W_{\mathrm{a}}(E, J)+W_{\mathrm{b}}(E, J)}\right]
$$

gegeben ist. $W_{\mathrm{a}}(E, J)$ ist die Anzahl offener Kanäle am Eingangskanal und entsprechend $W_{\mathrm{b}}(E, J)$ für den Ausgangskanal. Wenn die Reaktion über den Ausgangskanal sehr viel schneller als über den Eingangskanal ist, kann die Rückreaktion vernachlässigt werden und die Nettogeschwindigkeitskonstante geht in eine Einfangkonstante über, da $Y \rightarrow 1$. In allen anderen Fällen entscheidet Konkurrenz der Zerfallskonstanten über die Geschwindigkeit. Das Verhalten unterschiedlicher Übergangszustände, wie im Fall eines lockeren Eingangs- und festen Ausgangskanals, soll hier qualitativ erörtert werden. Die unterschiedliche Abhängigkeit der Rotationsbarrieren von $J$ ist bereits in Abschnitt 2.2.1 behandelt worden. Abbildung 2.4 zeigt die Konkurrenz schematisch: Mit steigender Rotationsquantenzahl nimmt die Barriere des effektiven Potentials beim festen Übergangszustand aufgrund der kompakteren Konfiguration stärker zu als beim lockeren, damit wird die Rückreaktion bei hoher Rotationsanregung wahrscheinlicher. $Y$ sinkt mit steigendem $J$, was zu einer negativen Temperaturabhängigkeit führen kann. Doch kann $J$ beim lockeren Eingangskanal mit höherer Potenz eingehen (Abschnitt 2.2.1), so dass sich dieser Effekt umkehren kann, die Nettogeschwindigkeit würde dann, in Abhängigkeit von $J$ betrachtet, ein Minimum durchlaufen. Dieser Effekt wird als „rotational channel switching“ bezeichnet [91].

Für die Energieabhängigkeit gibt es ebenfalls die Möglichkeit eines Wechsels zwischen bevorzugter Rückreaktion und Reaktion zu den Produkten. Die relative Höhe der Barrieren spielt in diesem Fall eine große Rolle. Die spezifische Geschwindigkeitskonstante des lockeren Eingangskanals steigt zunächst steil an, weist eine engere Krümmung auf und geht dann mit geringer Steigung weiter. Die Geschwindigkeitskonstante des festen Kanals steigt zwar flacher an, dafür aber über einen größeren Energiebereich. Es kommt, abhängig von der relativen Höhe der Barrieren zu einer 


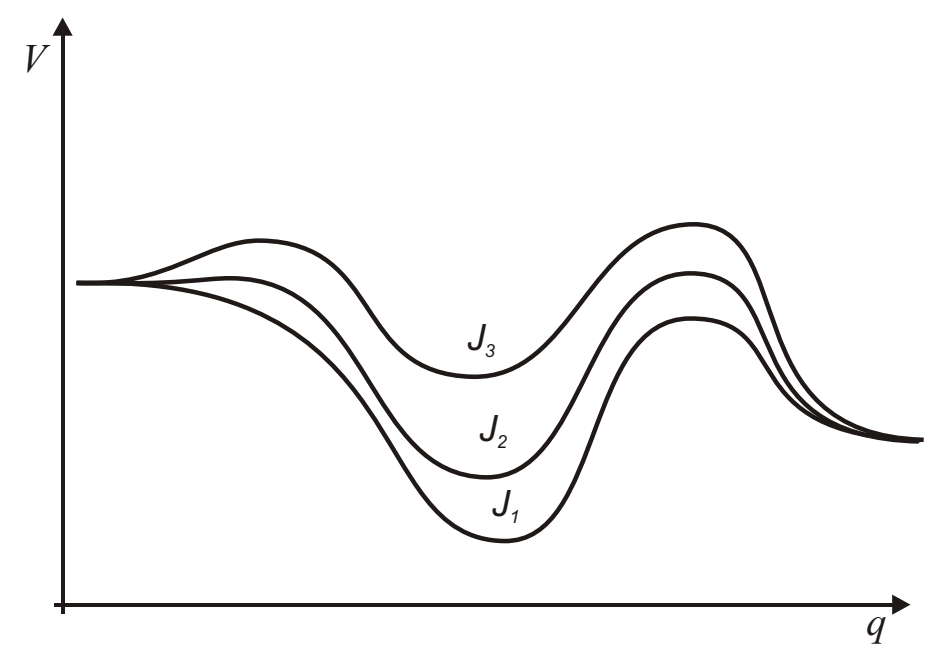

Abbildung 2.4: Rotational channel switching: Die Drehimpulsquantenzahl $J$ ändert das Verhältnis der Geschwindigkeitskonstanten über zwei Kanäle, es gilt $J_{1}<J_{2}<J_{3}$.

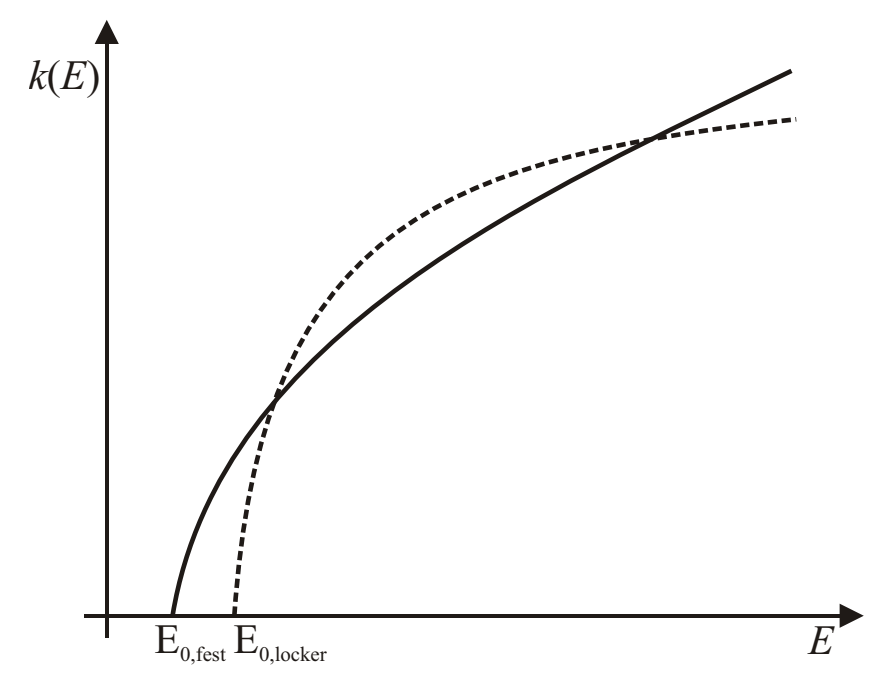

Abbildung 2.5: Die Energieabhängigkeit der Geschwindigkeitskonstanten führt zu einer Verschiebung der Reaktion zwischen bevorzugter Rückreaktion und bevorzugter Reaktion zu den Produkten. In dieser Auftragung ist die Barriere des festen Übergangszustandes $E_{0 \text {,fest }}$ niedriger als die des lockeren $E_{0, \text { locker }}$ 
Berührung oder zu einer doppelten Kreuzung der Kurven, wie in Abbildung 2.5 gezeigt. Für diesen Fall entsteht wieder ein Minimum in $Y(E, J)$, das sich auch in der thermischen Geschwindigkeitskonstante äußert. Dieser Kanalwechsel wird als „vibrational channel switching" bezeichnet.

Die starke negative Temperaturabhängigkeit vieler Radikalreaktionen, die mit der CRESU-Methode untersucht wurden, wäre mit diesem Modell plausibel erklärbar, vorrausgesetzt die beteiligten Komplexe können experimentell oder durch quantenmechanische Rechnungen gefunden werden.

Die Energieübertragung an ein Badgas hat in der bisherigen Betrachtung keine Rolle gespielt. Für den Niederdruckgrenzfall, wenn die Energieverteilung der Edukte unverändert bleibt und die Konkurrenz der unterschiedlichen Zerfallskanäle mit dieser Energieverteilung erfolgt, ist das korrekt. Ähnlich ist die Situation für den Fall einer thermischen Energieverteilung des Komplexes, in diesem Hochdruckgrenzfall ist eine explizite Behandlung des Energietransfers ebenfalls nicht erforderlich.

Liegt die Lebensdauer im Bereich der Stoßfrequenz eines Badgases, so wird eine Betrachtung der Energieübertragung an das Badgas nötig. Jetzt wird die Energieverteilung des Komplexes durch einen Mastergleichungsansatz bestimmt, für jedes Energieniveau des Komplexes konkurrieren die beiden Dissoziationskanäle mit der Energieübertragung, auch hier tritt eine Abhängigkeit von $J$ auf. Die Stöße beeinflussen schließlich über die veränderte Energieverteilung des Komplexes das Verzweigungsverhältnis.

In den beiden Grenzfällen für Hochdruck- und Niederdruckgrenzwert ist die Energieverteilung des Komplexes durch eine (verschobene) Boltzmann-Verteilung zu beschreiben. Die Stabilisierungsenergie des Komplexes gegenüber den Edukten spielt in diesem Zusammenhang keine Rolle.

Bei dem Komplex muss es sich nicht um ein kovalent gebundenes Molekül handeln, auch durch schwächere Wechselwirkungen wie Wasserstoffbrücken gebundene Spezies können analog behandelt werden, solange sie sich statistisch beschreiben lassen. Neben den Beispielen in Referenz [91] sind in jüngerer Zeit Arbeiten veröffentlicht worden, die Geschwindigkeitkonstanten für Reaktionen des OH-Radikals mittels der hier vorgestellten Vorgehensweise berechnen [27, 93, 94]. 


\subsection{Das Konzept der kinetically controlled selective fluorescence für $\mathrm{CHT} /$ Toluol}

Außer den Untersuchungen verschiedener Reaktionen des OH-Radikals ist die Lavaldüsen-Apparatur auch für Experimente zum Stoßenergietransfer nach der KCSFMethode (kinetically controlled selective fluorescence) [61] verwendet worden. Zustandsaufgelöste Experimente [95, 96] wurden vornehmlich an kleinen Molekülen durchgeführt. Experimente an Systemen mit hohen, quasikontinuierlichen Zustandsdichten, die gemittelte Größen wie die mittlere pro Stoß übertragene Energie $\langle\langle\Delta E\rangle\rangle$ in hoch schwingungsangeregten Molekülen zu ermitteln [97, 98], sind ebenfalls durchgeführt worden. Eine experimentelle Methode, die neben den Momenten des Energietransfers auch die Verteilungsfunktion des Energietransfers liefern kann, wurde von Luther et al. entwickelt [64, 62]. Die Methode wird als KCSI (kinetically controlled selective ionisation) bezeichnet. Die Terminologie legt bereits nahe, dass KCSI und KCSF sehr ähnlich sind. Beide Methoden sind für das System Cycloheptatrien/Toluol geeignet und bereits verwendet worden. Für Toluol soll der energieselektive Nachweis einer schwingungsangeregten Population dargestellt werden.

Abbildung 2.6 fasst die KCSF-Methode graphisch zusammen. Eine hoch schwingungsangeregte Population von Toluol-Molekülen wird durch die Photolyse von Cycloheptatrien (CHT) mit einem Laserpuls $\left(\lambda_{0}=248 \mathrm{~nm}\right)$ erzeugt. Dabei wird das CHT in den $\mathrm{S}_{1}$-Zustand angeregt. Eine innere Konversion gefolgt von einer schnellen Isomerisierung zum Toluol erzeugt schließlich die schwingungsheiße Population im elektronischen Grundzustand. Dieser Prozess ist schnell genug, um von der folgenden Schwingungsrelaxation durch Stöße mit dem Badgas M separiert werden zu können. Die Anfangsenergie des Ensembles $E_{0}$ ergibt sich als Summe der Isomerisierungsenthalphie $E_{\text {iso }}$ [99], der thermischen Energie der CHT-Moleküle und der Photonenenergie von $\lambda_{0} \mathrm{zu} 52100 \mathrm{~cm}^{-1}$.

Nach einer variablen Verzögerungszeit wird die relaxierende Population durch einen zweiten Laser $\left(\lambda_{1}=266.8-267.5 \mathrm{~nm}\right)$ in den $\mathrm{S}_{1}$-Zustand angeregt. Dort konkurriert die detektierte Fluoreszenz $\lambda_{\mathrm{F}}$ der Toluolmoleküle mit strahlungslosen Prozessen. Die Geschwindigkeitskonstanten beider Arten der Folgeprozesse sind von der Schwingungsanregung im elektronisch angeregten Zustand abhängig. Die Effi- 
zienz der IC steigt oberhalb einer Schwingungsenergie von $2130 \mathrm{~cm}^{-1}$ stark an [64]. Damit ist die Fluoreszenzausbeute kinetisch kontrolliert. Es entsteht ein begrenzter Energiebereich, aus dem Fluoreszenz stattfinden kann. Damit ist nur der Anteil des relaxierenden Toluolensembles sichtbar, der durch den zweiten Laserpuls in diesen Energiebereich verschoben wird. Der oft als Beobachtungsfenster bezeichnete Bereich kann als eine Funktion von Energie und Laserwellenlänge $W_{\mathrm{KCSF}}\left(E, \lambda_{1}\right)$ beschrieben werden. Eine genauere Diskussion der KCSF-Beobachtungsfenster von Toluol ist in Referenz [61] zu finden. Das zeitabhängige KCSF-Signal $I_{\mathrm{F}}(t)$ wird durch

$$
I_{\mathrm{F}}(t) \propto \int_{0}^{\infty} g(E, t) W_{\mathrm{KCSF}}\left(E, \lambda_{1}\right) \mathrm{d} E
$$

beschrieben, wobei $g(E, t)$ für die zeitabhängige Besetzung der Toluolmoleküle steht.

Die Analyse der gewonnenen Signale kann auf der Basis eines Mastergleichungansatzes mit genauer Kenntnis von Fensterfunktionen erfolgen und die Form der Stoßübergangswahrscheinlichkeitsfunktion $P\left(E, E^{\prime}\right)$ liefern, wie in Referenzen [64, 62] für die KCSI für viele verschiedene Stoßgase dargestellt.

Eine analytische Methode wie sie in Referenz [100] dargestellt ist, kann auf einfache Weise die mittlere pro Stoß übertragene Energie $\langle\langle\Delta E\rangle\rangle$ für den gesamten betrachteten Energiebereich liefern. Diese Daten sind ausreichend, um durch einen Vergleich anderer Messmethoden die Genauigkeit der in dieser Arbeit vorgestellten Ergebnisse zu diskutieren.

Allgemein kann der beobachtete Prozess durch eine Mastergleichung für die Besetzung jedes Niveaus beschrieben werden. Die Stoßprozesse sind bimolekulare Ereignisse, so dass die Änderung der Population eines Niveaus $n_{i}(t)$ durch

$$
\frac{\mathrm{d} n_{i}(t)}{\mathrm{d} t}=[\mathrm{M}] \sum_{j}\left[k_{i, j} n_{j}(t)-k_{j, i} n_{i}(t)\right]
$$

gegeben ist, wobei [M] die Konzentration des Badgases, $k_{i, j}$ und $k_{j, i}$ die Geschwindigkeitskonstanten der einzelnen Übergänge zwischen Niveaus $i$ und $j$ durch Stöße mit M bezeichnen. 

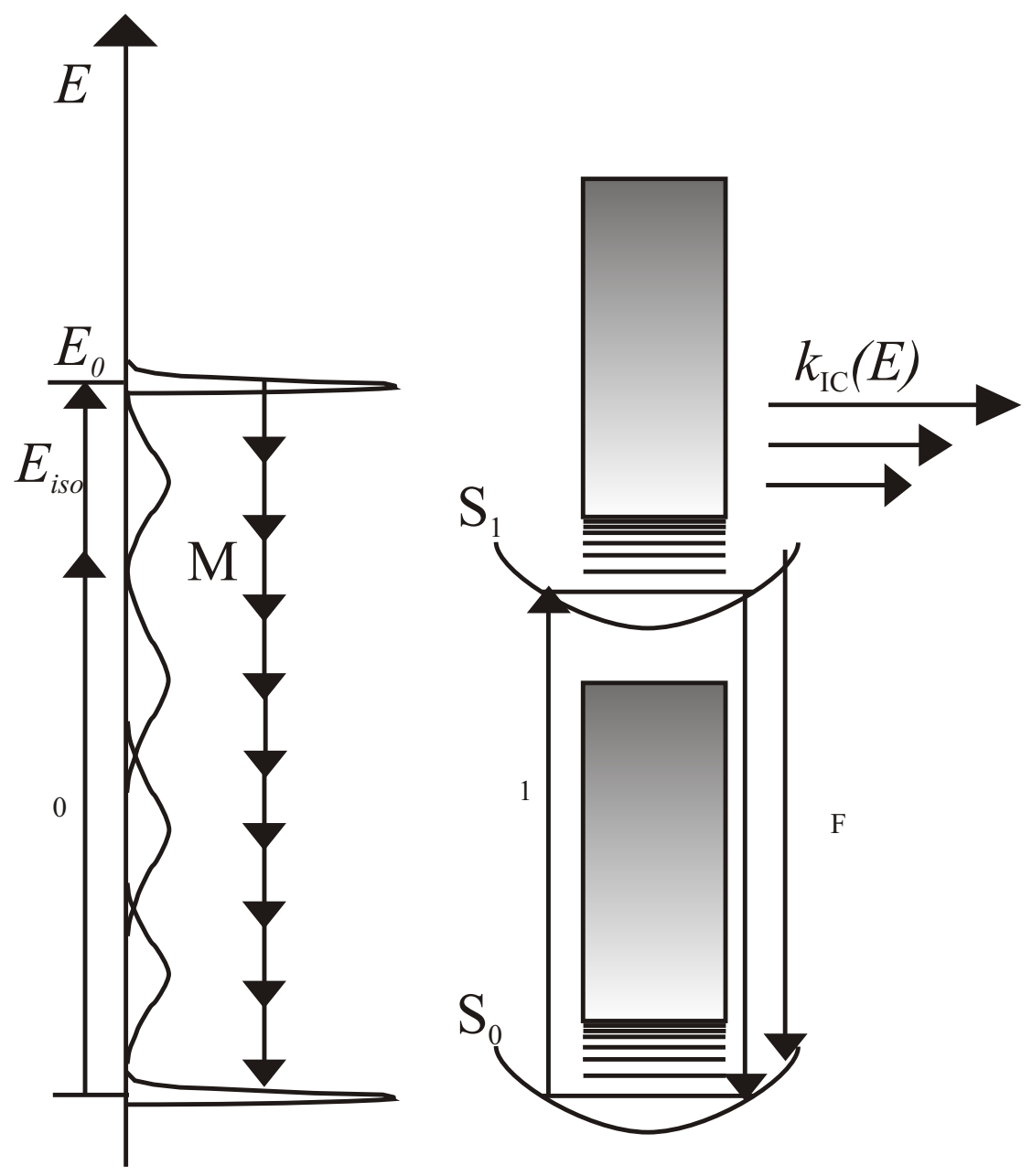

Abbildung 2.6: Die Methode der KCSF nutzt die Konkurrenz strahlungsloser Prozesse $k_{\mathrm{IC}}(E)$ im $\mathrm{S}_{1}$-Zustand von Toluol, um einen schmalen Energiebereich eines relaxierenden Ensembles zu detektieren. Nach der Anregung mit $\lambda_{0}$ isomerisiert Cycloheptatrien zu Toluol $\left(E_{\text {iso }}\right)$. Von der Anfangsenergie $E_{0}$ beginnt die Desaktivierung durch Stöße mit dem Badgas M. Je nach Probewellenlänge $\lambda_{1}$ wird ein anderer Energiebereich der relaxierenden Population, rechts dargestellt, als Fluoreszenzsignal $\left(\lambda_{\mathrm{F}}\right)$ abgebildet. 
Die Verbindung zwischen den zustandsspezifischen Geschwindigkeitskonstanten und einer experimentell zugänglichen Größe ist mit der Definition der Stoßzahl $Z$ als

$$
Z=\sum_{j} k_{i, j}
$$

hergestellt. Die Stoßzahl wird für die hier verwendeten Stoßpartner durch die Lennard-Jones-Stoßzahl angenähert, wobei die Potentialparameter aus Referenz [101] entnommen wurden. Für hohe Zustandsdichten geht die Formulierung in eine kontinuierliche Beschreibung über, die statt der Geschwindigkeitskonstanten eine stetige normierte Verteilungsfunktion $P\left(E, E^{\prime}\right)$ verwendet.

Die analytische Methode macht zunächst die Annahme, dass die Grenzen des Beobachtungsfensters durch eine Kastenfunktion wiedergegeben werden können. Die Fluoreszenswahrscheinlichkeit $V(E)$ lautet in diesem Fall

$$
V(E)=\left\{\begin{array}{lll}
0 & \text { wenn } & E>E_{\max }\left(\lambda_{1}\right) \\
V_{0} & \text { wenn } & E_{\min }\left(\lambda_{1}\right)<E<E_{\max }\left(\lambda_{1}\right) \\
0 & \text { wenn } & E<E_{\min }\left(\lambda_{1}\right)
\end{array}\right.
$$

mit der oberen Grenze $E_{\max }\left(\lambda_{1}\right)$ und der unteren Grenze $E_{\min }\left(\lambda_{1}\right)$.

Eine weitere Annahme betrifft die Form der Verteilung der relaxierenden Moleküle. Für eine $\langle\langle\Delta E\rangle\rangle$, die viel kleiner als die Breite der Verteilung ist, geht die Mastergleichung 2.29 in eine Diffusionsgleichung über. In diesem Fall geht die Besetzungsfunktion $g(E)$ für Poisson- oder Exponentialformen von $P\left(E, E^{\prime}\right)$ schon nach wenigen Stößen in eine Gaußfunktion über [102, 100]. Für die Auswertung der mittleren pro Stoß übertragenen Energie ist die Breite der Verteilung nicht relevant, damit kann die Annahme über die Form von $g(E)$ zur Forderung einer symmetrischen Verteilung verallgemeinert werden. $\langle\langle\Delta E\rangle\rangle$ kann direkt aus den Signalen abgelesen werden: Für Fenster, die die Boltzmannverteilung am Ende der Relaxation detektieren, kennzeichnet der halbmaximale Wert des Fluoreszenzsignals die Zeit, 
zu der der Schwerpunkt der Verteilung die obere Grenze des Beobachtungsfensters passiert. Die mittlere pro Stoß übertragene Energie ergibt sich dann zu

$$
\langle\langle\Delta E\rangle\rangle=\frac{E_{0}-E_{\mathrm{Fenster}}}{Z_{1 / 2}} .
$$

$E_{0}$ ist dabei die Anfangsenergie der Verteilung, $E_{\text {Fenster }}$ ist die obere Grenze des Beobachtungsfensters für monoton ansteigende Signale und $Z_{1 / 2}$ ist die Stoßzahl beim halbmaximalen Wert des Fluoreszenzsignals. 


\section{Kapitel 3}

\section{Experimentelles}

Beginnend mit einem kurzen Vergleich der freien Expansion und der Expansion durch eine Lavaldüse wird in den ersten Abschnitten der strömungstechnische Teil und die Konstruktion der Lavaldüsen erläutert. Daran schließt sich ein Überblick über den gesamten Aufbau an. Zuletzt wird die Datenverarbeitung und die Steuerung der Anlage erläutert.

\subsection{Isentrope Gasexpansionen}

Die vorliegende Arbeit stellt Ergebnisse zu kinetischen Messungen bei tiefen Temperaturen vor, deren Bedeutung für das Verständnis chemischer Reaktionen bereits in Kapitel 1 betrachtet wurde. Die Expansion eines Gases mit Überschallgeschwindigkeit ist eine Methode tiefe Temperaturen zu erzeugen, die in der Spektroskopie und der Untersuchung von Nukleationsprozessen bereits lange Anwendung findet. Oft handelt es sich dabei um so genannte freie Expansionen, also die Expansion durch ein Loch direkt ins Vakuum. Alternativ kann die Expansion aber auch durch Düsen mit ausgeformter Wandkontur erfolgen, eine besondere Form ist die Lavaldüse.

Die Abkühlung beruht auf der Umverteilung der zufälligen thermischen Bewegung der Gasteilchen in eine makroskopische, gerichtete Strömung. Da nur die Translationsenergie der Gasteilchen, nicht aber die inneren Freiheitgrade wie Rotation und Schwingung direkt gekühlt werden, ist die Abkühlung abhängig von der Zahl innerer Freiheitsgrade. Besonders effektiv ist sie für atomare Gase, was einer der Gründe 
ist, die Expansionen mit Helium oder Argon durchzuführen. Zur quantitativen Betrachtung ist der Adiabasiekoeffizient $\gamma$, definiert als

$$
\gamma=\frac{c_{\mathrm{P}}}{c_{\mathrm{V}}}
$$

wichtig, dabei ist $c_{\mathrm{P}}$ die molare Wärmekapazität bei konstantem Druck und $c_{\mathrm{V}}$ ist die molare Wärmekapazität bei konstantem Volumen.

Die Machzahl $M$ ist eine weitere Größe, die zur Beschreibung von Überschallströmungen verwendet wird, sie ist gegeben als

$$
M=\frac{\text { Flussgeschwindigkeit }}{\text { lokale Schallgeschwindigkeit }} .
$$

Eine Konsequenz der Strömung mit Überschallgeschwindigkeit ist, dass Störungen des Strömungsfeldes sich nicht gegen seine Flussrichtung ausbreiten können, da sie sich mit der jeweils lokalen Schallgeschwindigkeit durch ein Strömungsfeld bewegen. Bei der Berechnung der Wandkontur ist diese Eigenschaft sehr wichtig. Aus dem Alltag ist diese Eigenschaft als Überschallknall von Flugzeugen geläufig.

Thermodynamische Betrachtungen einer isentropen Expansion führen zu folgenden Beziehungen:

$$
\begin{aligned}
\frac{T}{T_{0}} & =\left(1+\frac{\gamma-1}{2} M^{2}\right)^{-1}, \\
\frac{\rho}{\rho_{0}} & =\left(\frac{T}{T_{0}}\right)^{1 /(\gamma-1)}, \\
\frac{p}{p_{0}} & =\left(\frac{T}{T_{0}}\right)^{\frac{\gamma}{\gamma-1}} .
\end{aligned}
$$

Die Temperatur T, die Dichte $\rho$ und der Druck $p$ im Gasstrahl der Düse sind durch $M$ und die Bedingungen im Stagnationsvolumen $\left(T_{0}, \rho_{0}, p_{0}\right)$ gegeben $[103,104]$. Bei gegebenem Gas und Ausgangsbedingungen hängen die thermodynamischen Größen im bewegten System also nur von der Machzahl ab. Ein solcher Fluss wird oft als eindimensionale Strömung bezeichnet. 
Die Betrachtung der eingeführten Größen zusammen mit der Eulerschen Bewegungsgleichung ermöglicht eine qualitative Aussage zur Form von Überschalldüsen. Die Änderung des Düsenquerschnitts $A$ steht in folgender Beziehung zur Strömungsgeschwindigkeit $v_{\mathrm{Fl}}$ :

$$
\begin{aligned}
\frac{\mathrm{d} A}{\mathrm{~d} p} & =\frac{A}{\rho v_{\mathrm{Fl}}^{2}}\left(1-M^{2}\right) \text { mit } \\
\frac{\mathrm{d} v_{\mathrm{Fl}}}{\mathrm{d} p} & >0 .
\end{aligned}
$$

$\rho$ bezeichnet die Dichte im Fluss [104]. Wird $A$ in Strömungsrichtung kleiner, so tritt eine Erhöhung von $v_{\mathrm{Fl}}$ auf, wenn $M<1$ ist. Wenn $M>1$ muss der Querschnitt in Strömungsrichtung wachsen, um eine Vergrößerung der Strömungsgeschwindigkeit zu erreichen. Ganz allgemein sollte eine Überschalldüse also eine konvergentdivergente Form besitzen. Eine Lochdüse ist daher in gewisser Weise die triviale Möglichkeit Gase auf Überschallgeschwindigkeit zu beschleunigen. Lochdüsen sind jedoch für kinetische Untersuchungen kaum anzuwenden: Die Machzahl wächst mit dem Abstand zur Düse an, so dass die Dichte und die Temperatur abfallen. Wird die Dichte so klein, dass nur noch selten Stöße zwischen den Gasteilchen stattfinden, sind die Freiheitsgrade der Teilchen voneinander unabhängig und die Ensembles sind nicht mehr thermisch. Die erreichbaren Temperaturen reichen von $20 \mathrm{~K}$ bis $1 \mathrm{~K}$ und die Dichte liegt unter $10^{15} \mathrm{~cm}^{-3}$.

\subsection{Die Lavaldüse}

Die Lavaldüse wurde Ende des 19. Jahrhunderts von Carl Gustav Patrik de Laval entwickelt um eine Dampfturbine mit Dampf zu versorgen. Später wurde die Lavaldüse beispielsweise bei Überschallwindkanälen, Triebwerken von Flugzeugen und als Steuerdüse für Satelliten genutzt. Bei der Anwendung in Windkanälen war die konstante Geschwindigkeit über einen großen Düsenquerschnitt entscheidend, beim Einsatz als Triebwerk ist die kollimierte Form des Strahls die entscheidende Eigenschaft für die Verwendung dieser Düsen. 
Die Expansion durch eine Lavaldüse ermöglicht eine konstante Machzahl nach dem Düsenausgang über mehr als zehn Düsendurchmesser. Aus den Gleichungen 3.3 und 3.4 ist ersichtlich, dass damit auch die Temperatur und Dichte im Strahl konstant bleiben. Zeitlich entspricht diese Strecke konstanter Bedingungen typischerweise einigen hundert Mikrosekunden, die beispielsweise als Reaktionszeit zur Verfügung stehen. Im Unterschied zur freien Expansion wird die Expansion in einen Niederdruckteil mit einem definierten Hintergrunddruck durchgeführt, der den Strahl einfasst. So sind Temperaturen von etwa $10 \mathrm{~K}-300 \mathrm{~K}$ und Teilchendichten von $10^{16} \mathrm{~cm}^{-3}-10^{18} \mathrm{~cm}^{-3}$ mit Lavaldüsen realisierbar. Der Einsatz der Lavaldüse als Tieftemperaturreaktor, wie er erstmals von Rowe et al.vorgestellt wurde, ist bereits in der Einleitung dargestellt worden. Dort lag der Schwerpunkt der Betrachtung auf den Ergebnissen, die mit dieser experimentellen Methode ermittelt wurden, in diesem Abschnitt erfolgt eine technische Darstellung der Methode.

\subsubsection{Berechnung der Wandkontur der Düsen}

Die besonderen Eigenschaften einer Gasexpansion durch eine Lavaldüse werden unter anderem durch ihre ausgeformte Wandkontur erreicht, daher wird die Berechnung der Wandkontur in einem eigenen Abschnitt betrachtet. Wie im vorangegangenen Abschnitt erwähnt hat eine Überschalldüse generell eine konvergent-divergente Form.

Im konvergenten Teil wird das Gas aus dem Stillstand auf Mach 1 beschleunigt. Für die Qualität der Überschallströmung ist der konvergente Teil zwar weniger wichtig als der divergente, allerdings sollte das Geschwindigkeitsprofil senkrecht zur Strahlachse möglichst konstant sein. Die Dicke der Grenzschichten, die sich durch die Viskosität des Gases an der Wand ausbilden, sollte minimiert werden. Daraus ergibt sich, dass der konvergente Teil kurz gegenüber dem divergenten sein sollte. Ein stetiger Übergang in den divergenten Teil ist ebenfalls sehr wichtig um ein Abreißen der Strömung von der Wand zu vermeiden. In der Praxis hat sich eine hyperbolische Form des konvergenten Teils bewährt, die mit geringer Steigung zum Düsenhals hin ausläuft. 


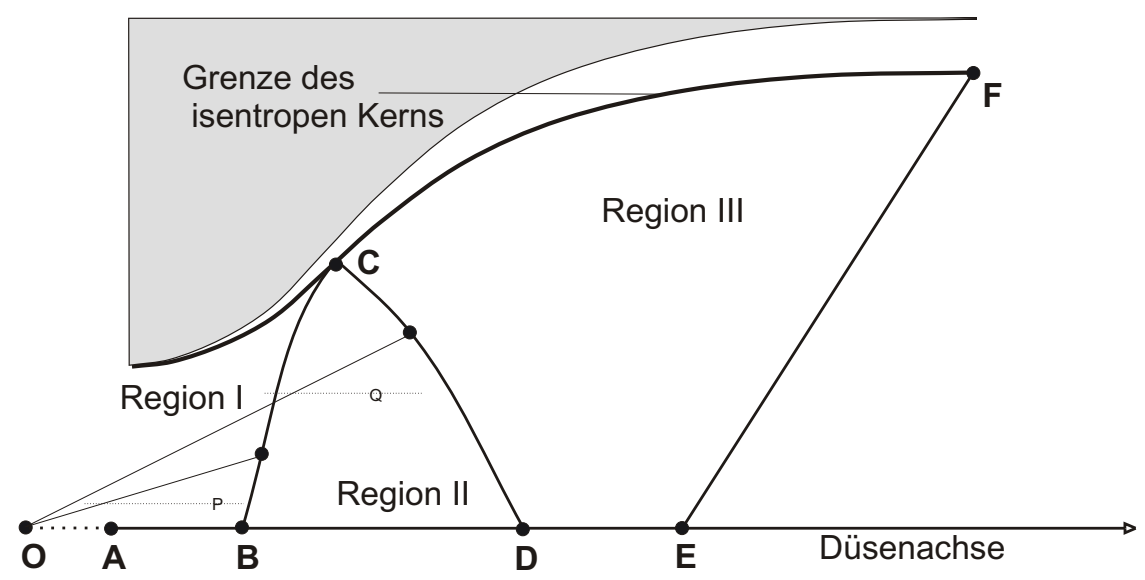

Abbildung 3.1: Der divergente Teil einer achsensymmetrischen Lavaldüse: die Konstruktion der Charakteristiken BC und $\mathbf{C D}$ wird illustriert. $\delta$ bezeichnet die Dicke der Grenzschicht, die Winkel $\theta_{\mathrm{P}}$ und $\theta_{\mathrm{Q}}$ werden im Text definiert. Grau unterlegt ist der Düsenkörper dargestellt.

Im divergenten Teil wird die Strömung von Mach 1 auf den endgültigen konstanten Wert beschleunigt, was eine S-förmige Wandkontur bedingt. Die Beschleunigung erzeugt eine Reihe von Expansionswellen, die den Ausgang der Düse allerdings nicht überschreiten dürfen, um die konstante Machzahl zu gewährleisten. Der divergente Teil wird mit Hilfe eines Programms von M. Smith [104], mit einer mehrstufigen Iterationsmethode berechnet. Der Quelltext des Quickbasic Programms ist in Referenz [103] veröffentlicht. Eine Beschreibung der Berechnung findet sich auch in Referenz [105].

Anhand kurzer Stichpunkte wird die gesamte Prozedur dargestellt, bevor jeder Punkt einzeln in größerem Detail besprochen wird:

- Festlegen der Eingabeparameter:

- Machzahl am Ausgang der Düse

- Dichte des Strahls

- Adiabasiekoeffizient des expandierten Gases

- Machzahl und Strömungswinkel zur Strahlachse am Wendepunkt der Wandkontur 
- Halsdurchmesser und die Anzahl der Charakteristiken in den Regionen I und III

- Berechnung der Lage wichtiger Punkte auf der Strahlachse und des Wendepunkts der Kontur anhand der Eingabedaten.

- Optimierung der berechneten Geometrie durch Änderung der eingangs verwendeten Formparameter

- Berechnung des isentropen Kerns der Expansion

- Berechnung der Grenzschicht

Die Temperatur und Dichte des Düsenstrahls und das verwendete Gas werden bereits bei der Konstruktion festgelegt (vgl. Gl.3.3 und 3.4). Die verbleibenden Einstellmöglichkeiten sind lediglich der Druck im Stagnationsvolumen $p_{0}$ und in der Vakuumkammer $p_{\text {bg }}$ (der Hintergrunddruck) worauf im Abschnitt 3.2 .2 näher eingegangen wird. Für jede Temperatur wird eine eigene Lavaldüse benötigt.

Der divergente Teil kann also zunächst in den isentropen Kern, das eigentliche Überschallströmungsfeld, und die Grenzschicht nahe der Wand der Düse unterteilt werden. Die Grenzschicht enthält Moleküle, die durch Stöße mit der Wand der Düse nicht mehr zum isentropen Ensemble gehören, da sie Impuls und kinetische Energie mit der Wand ausgetauscht haben. Die Berechnung des Überschallströmungsfeldes erfolgt nach der Methode der Charakteristiken von Moger und Ramsay. Der isentrope Kern wird in drei Regionen unterteilt (siehe Abbildung 3.1): In der Region I wird in einer starken Vergrößerung des Düsenquerschnitts etwa die Hälfte der endgültigen Machzahl erreicht. Am Wendepunkt der S-förmigen Wandkontur endet diese Region und Region II schließt sich an. Hier herrscht Quellfluss, ähnlich dem Fluss einer freien Expansion: der Abstand von einem Quellpunkt O, aus dem die Strömung radial zu entspringen scheint, legt die Machzahl fest. Die Methode der Charakteristiken ist in dieser Region der Düse also zur Berechnung des Strömungsfeldes nicht nötig. Abschließend werden in Region III die Verdünnungsstöße aus dem Strömungsfeld entfernt, damit die Machzahl einen konstanten Wert annehmen kann.

Zum besseren Verständnis der folgenden Ausführungen bietet sich ein kurzer Exkurs über die Methode der Charakteristiken und die Eigenschaften von Überschall- 


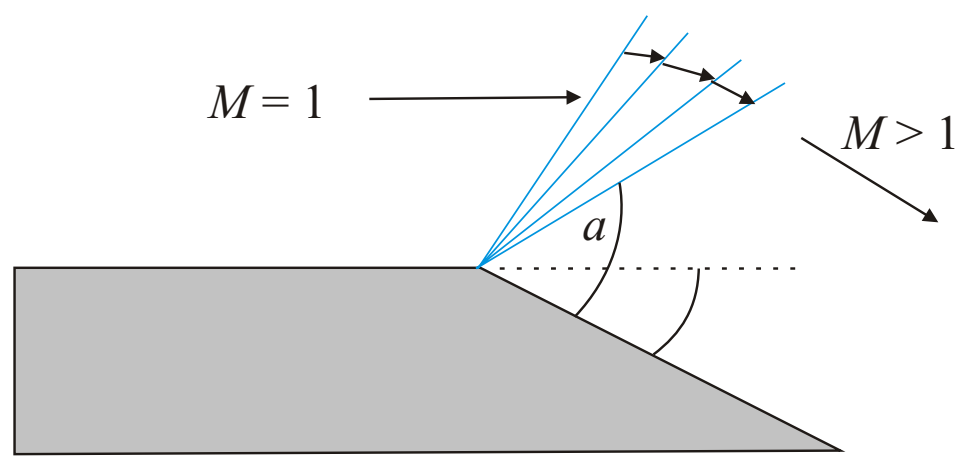

Abbildung 3.2: Illustration eines Prandtl-Meyer-Expansionsfächers: Die Strömung mit $M=1$ wird in schwachen Verdünnungsstößen unter Änderung der Strömungsrichtung auf $M>1$ beschleunigt. Die Charakteristik dieser Störung breitet sich mit dem Winkel $a$ im Strömungsfeld aus.

strömungen allgemein an. Anhand der Abbildung 3.2 soll der Begriff Charakteristik erklärt werden: Dargestellt ist ein Strömungsfeld mit $M=1$ das auf eine scharfe Winkeländerung der Wand trifft. Die Machzahl und die Richtung der Strömung nach dieser Störung sollen berechnet werden. Da in Überschallströmungen keine Information über diese Störung stromaufwärts transportiert werden kann, entspringt an der abrupten Wandkrümmung um den Winkel $\Psi$ ein Expansionsfächer in das Strömungfeld. Innerhalb der Grenzen dieses Expansionsfächers ereignet sich eine Reihe von Verdünnungsstößen, die am Ende des Fächers abgeschlossen sind. Das stromabwärtige Ende des Fächers ist eine Charakteristik, die sich im Winkel $a$ in das Strömungsfeld ausbreitet. Grundsätzlich ist eine Charakteristik eine geometrische Grenze an der sich Zustandsgrößen einer Strömung um einen endlichen Betrag ändern. Je kleiner die Winkeländerung ist, desto enger wird der Winkel den der Expansionsfächer überspannt. Für eine infinitesimal kleine Winkeländerung gilt die Beschreibung durch Charakteristiken daher exakt. Die Wandkontur der Düse wird also in viele infinitesimale Winkeländerungen der Wand zerlegt, für die die Annahme einer endlichen Änderung an einer Grenzlinie — also einer Charakteristik gut erfüllt ist. Um die Richtung des Flusses und die Geschwindigkeit in den Regionen I und III zu berechnen, werden die dazu benötigten Größen eingeführt. Die 
Machzahl nach einer Winkeländerung der Wand lässt sich mit Hilfe des PrandtlMeyer-Winkels $\Psi$ beschreiben:

$$
\Psi=\sqrt{\frac{\gamma+1}{\gamma-1}} \arctan \left(\sqrt{\left(M^{2}-1\right) \frac{\gamma-1}{\gamma+1}}\right)-\arctan \left(\sqrt{M^{2}-1}\right)
$$

In einer achsensymmetrischen Düse ist es offensichtlich, dass sich von verschiedenen Änderungen der Wandwinkel hervorgerufene Charakteristiken kreuzen. Die Änderungen der Strömungsbedingungen verhalten sich in diesem Fall additiv. Das achsensymmetrische Strömungsfeld soll mit Zylinderkoordinaten $z$ für die Entfernung auf der Achse vom Ursprungspunkt $\mathbf{O}$ und $r$ für die radiale Entfernung beschrieben werden. Für die Änderung der Strömungsrichtung und -geschwindigkeit durch eine Wandkrümmung werden folgende Differenzialgleichungen erhalten.

$$
\begin{aligned}
\frac{\mathrm{d} r}{\mathrm{~d} z} & =\tan (\theta a) \\
0 & =\mathrm{d} \theta \pm \frac{\cot (a)}{W} \mathrm{~d} W \frac{\sin (\theta) \sin (a)}{\sin (\theta+a)} \frac{\mathrm{d} r}{r} \text { wobei } \\
W^{2} & =\left(\frac{V}{V_{\max }}\right)^{2}=\frac{\gamma-1}{2} M^{2}\left(1+\frac{\gamma-1}{2} M^{2}\right)^{-1} \\
V_{\max } & =\sqrt{\frac{2 \gamma}{\gamma-1} \frac{k_{\mathrm{B}} T_{0}}{m}} \\
a & =\sin ^{-1}(1 / M)
\end{aligned}
$$

$\theta$ ist der Winkel der Strömungsrichtung bezüglich der Düsenachse, $a$ wird als Machwinkel bezeichnet, $W$ ist das so genannte Geschwindigkeitsverhältnis, definiert als das Verhältnis der thermodynamischen Grenzgeschwindigkeit zur Strömungsgeschwindigkeit. $W$ ist als Funktion von $M$ nur zur bequemeren Darstellung der obenstehenden Differenzialgleichungen in Zylinderkoordinaten eingeführt. In der Definition von $V_{\max }$ steht $k_{\mathrm{B}}$ für die Boltzmann-Konstante, $m$ für die Masse der Gasteilchen und $T_{0}$ für die Temperatur im Stagnationsvolumen. Die Gleichungen 3.9 und 3.10 werden mit endlichen Differenzen dazu verwendet, die Strömungsbedingungen entlang der Charakteristiken zu berechnen. 
Bei der Methode von Moger und Ramsay [106] werden aus den Eingangsparametern zunächst die Grenzen der Region II mit Hilfe der entsprechenden Prandtl-Meyer Winkel gemäß

$$
\begin{aligned}
\Psi_{\mathrm{P}} & =\Psi_{\mathrm{B}}+2 \theta_{\mathrm{P}} \\
\theta_{\mathrm{P}} & =1 / n \cdot \eta \ldots n / n \cdot \eta \\
\Psi_{\mathrm{Q}} & =\Psi_{\mathrm{C}}+2\left(\eta-\theta_{\mathrm{Q}}\right) \\
\theta_{\mathrm{Q}} & =\eta-1 / n \cdot \eta \ldots 0 \\
n & \in \mathbf{N}
\end{aligned}
$$

berechnet. Die Konstruktion der Region II ist in Abbildung 3.1 dargestellt. $\Psi_{\mathrm{P}}$ und $\Psi_{\mathrm{Q}}$ stehen für den Prandtl-Meyer-Winkel an den Punkten $\mathbf{P}$ und $\mathbf{Q}$. Die Winkel $\theta_{\mathrm{P}}$ bzw. $\theta_{\mathrm{Q}}$ bezeichnen die Flusswinkel bezüglich der Düsenachse. Sie sind Winkelsegmente des Winkels AOC. $\Psi_{\mathrm{B}}$ und $\Psi_{\mathrm{C}}$ sind die Prandtl-Meyer-Winkel an den entsprechenden Punkten, $\Psi_{\mathrm{B}}$ ist durch die Machzahl bei B, einem Eingabeparameter, gegeben. Der Winkel des Flusses am Punkt $\mathbf{C}$ wird mit $\eta$, ebenfalls ein Eingabeparameter, bezeichnet. Analog zu den Punkten $\mathbf{P}$ und $\mathbf{Q}$ werden über die Variation von $\theta$ weitere Punkte auf den Charakteristiken BC und CD erstellt, womit die Grenzen der Region II festgelegt sind.

Innerhalb Region II ist der Fluss nun ebenso festgelegt, da hier Quellfluss herrscht und die Strömung radial aus $\mathbf{O}$ entspringt. Die Machzahl ist durch die Entfernung vom Punkt $\mathbf{O}$ gegeben.

Die Charakteristik EF ist eine gerade Linie, die Region III begrenzt. Ihre Länge wird über den Massefluss durch die Düse mit Hilfe einer parabolischen numerischen Integration festgelegt (parabolisch, weil zur Berechnung eines neuen Punktes drei bekannte Punkte verwendet werden, die eine quadratische Gleichung festlegen). Das Verhältnis vom Ausgangsdurchmesser zum Halsdurchmesser $\frac{A}{A *}$ bestimmt den Düsenquerschnitt, damit sind auch die Grenzen von Region III festgelegt.

Die Berechnung der Charakteristiken in den Regionen I und III bestimmt die Wandpunkte des isentropen Kerns. Der Verlauf der Machzahl auf der Düsenachse ist bereits aus den Eingabeparametern berechenbar und so werden die Punkte an den Grenzen von Region II und auf der Düsenachse verwendet um das Strömungsfeld 
iterativ in den beiden anderen Regionen bis zur Wand hin zu berechnen. Tatsächlich werden die Charakteristiken noch weiter nach außen berechnet, die genaue Festlegung der Lage der Wandpunkte erfolgt durch Integration des Masseflusses entlang der entsprechenden Charakteristiken.

Beginnend an den bereits festgelegten Punkten B bzw. D werden mit Hilfe der Gleichungen 3.9 und 3.10 in kleiner Entfernung zu den bekannten Punkten, unter Berücksichtigung der Werte der ersten Ableitung für Flussgeschwindigkeit und Richtung, weitere bestimmt. Zur Berechnung eines Netzes aus solchen Punkten werden die Achsenpunkte und die Punkte auf den Charakteristiken BC, CD und EF verwendet. Der isentrope Kern von Lavaldüsen ist am Ende dieser Iteration genau festgelegt.

Die Bestimmung der Dicke der Grenzschicht $\delta$, die zum Radius des isentropen Kerns addiert werden muss, um die physikalische Wand der Düsen festzulegen, erfordert die Eingabe weiterer Daten. Die Transporteigenschaften des Gases, die Temperatur im Stagnationsvolumen und der Wände gehen in einem empirischen Verfahren ein, um die Dicke der Grenzschicht zu bestimmen. Diese wird benötigt, um das Überschallregime im isentropen Kern thermisch und kinematisch von der physikalischen Wand zu isolieren. Der insentrope Kern legt den inneren Radius der Grenzschicht fest, die senkrecht zur Grenze des isentropen Kerns addiert wird. Die Strömung in der Grenzschicht wird als laminar angenommen. Das Verfahren stützt sich auf empirische Methoden der Aerodynamik, die wenige Rückschlüsse auf die zugrundeliegende Physik zulassen. An dieser Stelle wird nur auf die Dissertation von D. B. Atkinson [103] und dort genannte Quellen verwiesen.

Als Ergebnis dieser Berechnungen wird ein Satz zylindrischer Koordinaten erhalten. Die im Experiment verwendeten Lavaldüsen wurden nach den Koordinaten (ca. 300 Punkte) mit einer CNC-Fräse in der Feinmechanikwerkstatt des Instituts für Physikalische Chemie in Göttingen aus Aluminium gefertigt.

Die so konstruierten Düsen können unter geeigneten Betriebsbedingungen Überschallstrahlen mit konstanter Temperatur und Dichte auf einer Länge von mindestens $10 \mathrm{~cm}$ liefern. Durch die vorgegebene Dichte im Überschallstrahl ist ein Bereich von Drücken vor und nach der Expansion eingegrenzt, innerhalb dessen die Düse optimal arbeitet. Wie die besten Betriebsbedingungen gefunden werden und 
die Konstanz der Bedingungen überprüft wird, ist im folgenden Abschnitt 3.2.2 erläutert.

\subsubsection{Bestimmung der Bedingungen im Überschallstrahl}

Nach der Fertigung der Düsen und bei jedem Wechsel der Düsen werden die Bedingungen $(T, \rho, p)$ im Strahl der Lavaldüsen überprüft und die Betriebsbedingungen anhand der Variation des Druckes im Stagnationsvolumen und des Hintergrunddrucks optimiert.

Zur Bestimmung der thermodynamischen Bedingungen im Überschallstrahl werden zwei komplementäre Verfahren eingesetzt. Die Besetzung der Rotationszustände des OH-Radikals bietet eine Möglichkeit die Temperatur im Strahl der Lavaldüse zu messen. Eine andere Möglichkeit ist durch Druckmessungen mit einem PitotStaurohr gegeben.

Die Messung der Rotationsbesetzung hat den Vorteil, das keine Annahmen über die Strömung in die Berechnung der Temperatur eingehen und auch nicht-thermische Ensembles detektiert werden können. Ein weiterer Vorteil der Methode ist die hohe Zeitaufösung mit der die Besetzung der OH-Radikale untersucht werden kann. Das Spektrum des OH-Radikals ist bereits gut untersucht und verstanden. In der Dissertationsschrift von Tim Spangenberg [34] finden sich detaillierte Ergebnisse zur Relaxation der OH-Radikale, die Aufschluss darüber geben, wie schnell der Überschuss der Photolyseenergie in das Badgas verteilt wird. Zur Bestimmung der Temperatur im bewegten System des Expansionsstrahls wird ein rotationsaufgelöstes Anregungsspektrum des OH-Radikals bei konstanter Zeitverzögerung zwischen Erzeugung der Radikale und Anregung zur Fluoreszenz aufgenommen. Aufgrund der starken Spin-Bahn Kopplung stehen beim OH-Radikal zwölf unterschiedlich intensive Zweige zur Verfügung. Die Termenergien und die Überganswahrscheinlichkeiten können aus Referenz [86] entnommen werden. Ein Zweig stellt dabei eine Serie von Übergängen dar, die sich nur in der Rotationsquantenzahl unterscheiden. Der Unterschied in der Intensität der Linien $I_{\mathrm{nm}}$ eines Zweiges ist bei konstanter 
Lichtintensität durch die Besetzung im Ausgangszustand $N_{\mathrm{n}}$ und der Übergangswahrscheinlichkeit $A_{\mathrm{nm}}$ gegeben.

$$
I_{\mathrm{nm}}=N_{\mathrm{n}} A_{\mathrm{nm}}
$$

Die Besetzung der Ausgangsniveaus kann durch eine Boltzmann-Verteilung beschrieben werden, wenn das Ensemble thermisch equilibriert ist. Dies ist im Überschallstrahl der Lavaldüse schon einige Mikrosekunden nach der Photolyse der Fall [34]. Für diesen Fall lautet die Boltzmann-Verteilung

$$
\frac{N_{\mathrm{n}}}{N_{0}}=\exp \left(\frac{-E_{\mathrm{n}}}{k_{\mathrm{B}} T}\right)
$$

mit der Boltzmann Konstante $k_{\mathrm{B}}$ und der Rotationsenergie des Zustands $n E_{\mathrm{n}}$. In einer Auftragung von $\ln \frac{I_{\mathrm{nm}}}{A_{\mathrm{nm}}}$ gegen $E_{\mathrm{n}}$ erhält man aus der Steigung der Geraden

$$
\ln \frac{I_{\mathrm{nm}}}{A_{\mathrm{nm}}}=\mathrm{const}-\frac{E_{\mathrm{n}}}{k_{\mathrm{b}} T}
$$

die Temperatur. Durch Verschiebung der Düse gegenüber der LIF-Optik kann die Temperatur entlang des Strahls bestimmt werden.

Die Messung der Temperatur mit einem Pitot-Rohr hat gegenüber der spektroskopischen Messung den Vorteil, dass sie mit geringem Aufwand durchzuführen ist. Sie verfügt zwar nicht über die hohe Zeitauflösung wie die spektroskopische Methode, kann aber die Bedingungen in hoher Ortsauflösung bestimmen, so sind nicht nur die Bedingungen in der Achse der Laser zugänglich, sondern auch die radiale Ausdehnung des Strahls kann untersucht werden. Aus den Gleichungen 3.3 und 3.4 ist ersichtlich, dass die Machzahl, bei gegebenem Gas und bekannten Ausgangsbedingungen, die Temperatur und Dichte einer isentropen Gasexpansion festlegt. Die 


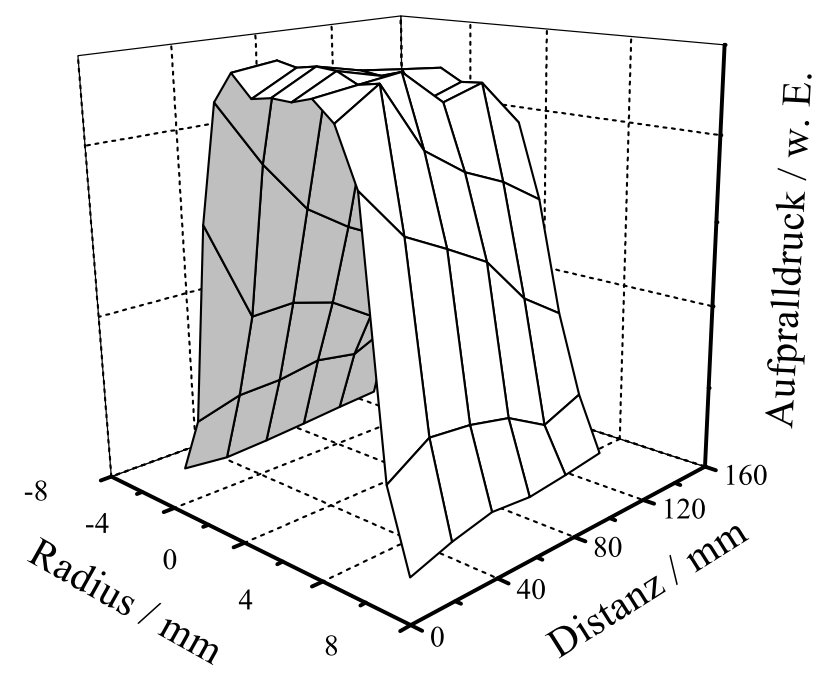

Abbildung 3.3: Dreidimensionale Darstellung des Aufpralldrucks gegen die radiale und axiale Entfernung von der Lavaldüse M358E16

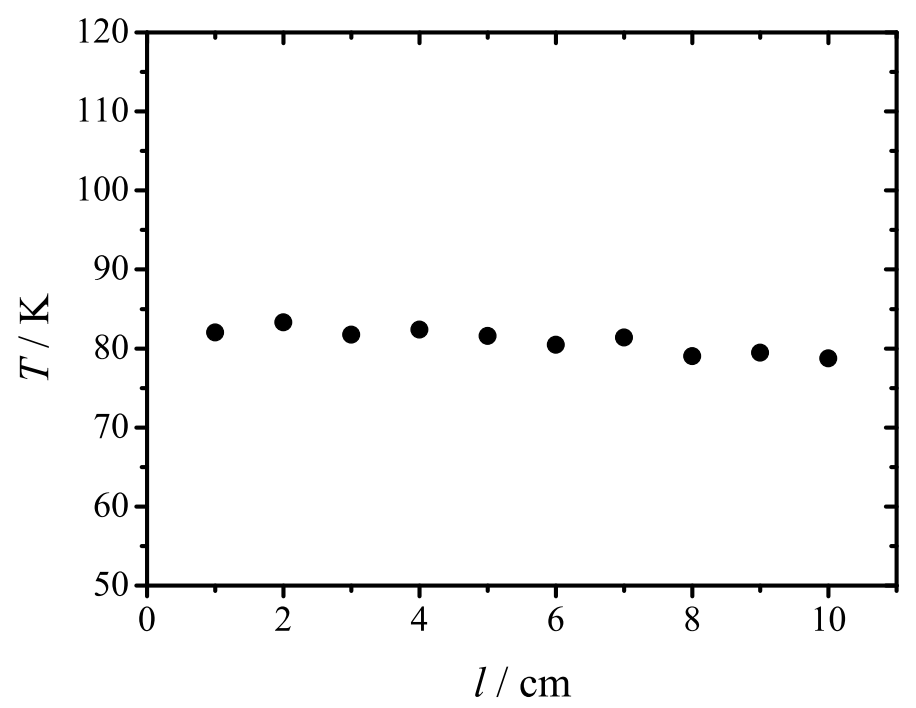

Abbildung 3.4: Messungen der Temperatur im Strahl der Lavaldüse durch ein Pitot-Rohr für die Düse M358E16 
Messung des Aufpralldrucks des isentropen Gasstrahls $p_{\mathrm{i}}$ (Honeywell 40PC001B) ist über die Rayleigh-Pitot Formel

$$
\frac{p_{\mathrm{i}}}{p_{0}}=\left[\frac{(\gamma+1) M^{2}}{(\gamma+1) M^{2}+2}\right]^{\frac{\gamma}{\gamma-1}}\left[\frac{\gamma+1}{2 \gamma M^{2}-(\gamma-1)}\right]^{\frac{1}{\gamma-1}} .
$$

mit der Machzahl verknüpft. Die Messung des Anfangsdrucks im Stagnationsvolumen $p_{0}$ erfolgt durch einen Druckaufnehmer (Omega PX170-28DV bzw. PX26005GV), siehe Abbildung 3.6. Bei den hier verwendeten Düsen liegt $p_{0}$ zwischen etwa 40 mbar für die wärmste Düse (Machzahl:2.1) und etwa 140 mbar für die kälteste Düse (Machzahl:5.0). Eine Messung des Aufpralldrucks an verschiedenen Punkten längs des Gasstrahls bei gleichzeitiger Bestimmung des Stagnationsdrucks liefert die Temperatur entlang der Strahlachse wie in Abbildung 3.4 dargestellt. Eine dreidimensionale Druckspur ist in Abbildung 3.3 als Auftragung des Aufpralldrucks gegen die radiale Entfernung von der Achse und entlang der Strahlachse dargestellt.

Die Lavaldüsen können jeweils einen schmalen Bereich von Dichte und Temperatur über eine hinreichend große Strecke konstant erzeugen. Innerhalb dieses Bereichs hängen die Bedingungen von den exakten Werten der Drücke im Stagnationsvolumen und in der Vakuumkammer ab. Die bei den einzelnen Experimenten verwendeten Bedingungen sind im Kapitel 4 bei den jeweiligen Reaktionen in Tabellen dargestellt. 


\subsection{Versuchsanordnung der Lavaldüsen-Apparatur}

Dieser Abschnitt liefert eine Beschreibung des optischen und vakuumtechnischen Aufbaus der Apparatur, in der die Ergebnisse dieser Arbeit gemessen wurden. Abbildung 3.5 zeigt eine schematische Darstellung der Apparatur, die zeitaufgelöste Messungen laserinduzierter Fluoreszenz in einer kalten gepulsten Überschallexpansion durch eine Lavaldüse ermöglicht. Der Aufbau beginnt mit der Beschreibung des experimentellen Aufbaus zur Detektion des Hydroxylradikals, die apparativen Änderungen für die Detektion von schwingungsangeregtem Toluol folgen in einem eigenen Abschnitt.

\section{Optischer Aufbau}

Zum Nachweis der OH-Radikale im Schwingungsgrundzustand ( $\mathrm{v}=0$ ) und schwingungsangeregter $\mathrm{OH}-\mathrm{Radikale}(\mathrm{v}=1)$ wird laserinduzierte Fluoreszenz benutzt. Die Erzeugung der Radikale erfolgt durch photolytische Spaltung von Wasserstoffperoxid. Die Zeitauflösung wird durch die zeitliche Verschiebung des Photolyselasers gegenüber dem LIF Laser erreicht. Bei dem Photolyselaser handelt es sich um einen Excimerlaser (EMG Lambda Physik) der mit verschiedenen Gasmischungen betrieben wird. Beim Betrieb mit KrF (120 mbar $5 \%$ Fluor-Helium-Mischung, 150 mbar Krypton und 2230 mbar Helium) erzeugt dieser Laser 12 ns lange Laserpulse bei $\lambda=248 \mathrm{~nm}$. Dabei werden die OH-Radikale ausschließlich im Schwingungsgrundzustand erzeugt. Die Fluoreszenz der OH-Radikale (v $=0)$ wird durch die Pulse (7ns, $\lambda=281.915 \mathrm{~nm}, 0.25 \mathrm{~mJ}$ ) der zweiten Harmonischen eines optisch gepumpten Farbstofflasers (Lambda Physik Scanmate 2E) mit Rhodamin 6G (Ethanol) angeregt. Dabei wird die $Q_{1}(1)$-Linie des $(0 \rightarrow 1)$ Übergangs verwendet. Als Pumplaser für den Farbstofflaser dient ein Nd:YAG-Laser (Spectra-Physics Quanta Ray GCR-150) mit interner Frequenzverdopplung $(\lambda=532 \mathrm{~nm}, 70 \mathrm{~mJ})$. Die Erzeugung von schwingungsangeregten $\mathrm{OH}$-Radikalen $(\mathrm{v}=1)$ erfolgte durch die Photolyse von Wasserstoffperoxid mit $\lambda=193 \mathrm{~nm}$, die im Excimerlaser erzeugt wurden. Dazu wurde eine Gasmischung aus 160 mbar einer Fluor-Helium Mischung (5\%), 300 mbar Argon mit Helium auf insgesamt 2200 mbar aufgefüllt. Der Farbstofflaser wird auf 


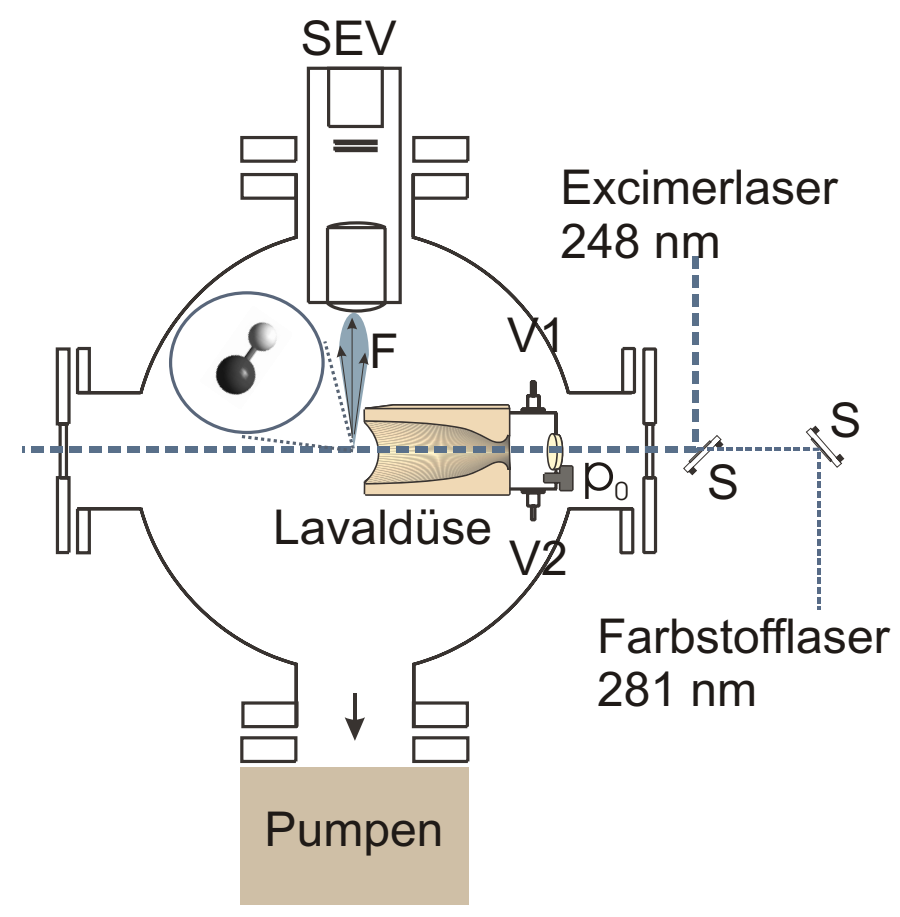

Abbildung 3.5: Ein schematisierter Überblick über den experimentellen Aufbau der Lavalapparatur: SEV bezeichnet den Sekundärelektronenvervielfacher, F die Fluoreszenz. Die Einlassventile sind durch V1 und V2 und der Druckaufnehmer für das Stagnationsvolumen mit $\mathrm{p}_{0}$ gekennzeichnet. Mit S werden die Spiegel bezeichnet.

die Wellenlänge $288.233 \mathrm{~nm}$ eingestellt, um die $\mathrm{Q}_{1}(1)$-Linie des $(1 \rightarrow 2)$-Übergangs anzuregen.

In Abbildung 3.5 sind die Strahlengänge der beiden Laser als gestrichelte Linien gezeichnet. Sie verlaufen also kollinear in der Längsachse der Expansion und werden durch ein Fenster in der Rückwand des Stagnationsvolumens durch die Düse hindurch bis zu einem Brewsterfenster am Ende der Vakuumkammer geleitet. Senkrecht zu den Laserstrahlen ist im Zentrum der Vakuumkammer eine Sammellinse (Quarz, $\mathrm{f}_{1}=50 \mathrm{~mm}$ ) angebracht, um die Fluoreszenz F über eine weitere Linse $\left(\mathrm{f}_{2}=150 \mathrm{~mm}\right)$ und ein optisches Filtersystem bestehend aus einem UV Kurzpassfil- 
ter (UG11) und einen Interferenzfilter $\left(\lambda_{0}=308 \mathrm{~nm}, T_{\max }=0.37\right.$, FWHM $\left.=16 \mathrm{~nm}\right)$ auf einem Sekundärelektronenvervielvacher SEV (Thorn EMI 9413QB) zu detektieren. Die resultierende Spannung am SEV wird in einem Boxcar Integrator (EG\&G Parc 4121B) über $150 \mu$ s integriert und in einen Rechtecksspannungspuls umgerechnet, der zur weiteren Datenverarbeitung über eine A/D-Wandlerkarte (Data Translation) in einen PC eingelesen wird. 30 - 50 Einzelschüsse der beiden Laser werden gemittelt um das Signal/Rausch Verhältnis zu verbessern, danach wird die Verzögerungszeit zwischen den beiden Lasern um $1 \mu$ s verschoben und ein weiterer Datenpunkt aufgenommen.

\section{Vakuumtechnischer Aufbau}

Die Expansion zur Erzeugung tiefer Temperaturen, sowie die Raumtemperaturmessungen wurden in einer Edelstahl-Vakuumkammer (6-fach CF-250 Kreuz) durchgeführt. Wie bereits an anderer Stelle erwähnt, wird die Expansion durch die Lavaldüse gepulst betrieben. Der gepulste Betrieb der Apparatur ermöglicht bei der verwendeten Taktrate und Öffnungszeit die Reduzierung des Gasstroms um mehr als eine Größenordnung. Damit konnte das Vakuum mit einer Pumpenkombination aus einer Wälzkolben- (BOC Edwards EH500) und einer Drehschieberpumpe (BOC Edwards E2M80) aufrecht erhalten werden. Die Gesamtkapazität der Pumpen beträgt $400 \mathrm{~m}^{3} / \mathrm{h}$ und erreicht im gasballastfreien Betrieb einen Druck von etwa $10^{-5}$ bar. An einem der Flansche der Vakuumkammer ist ein Feindosierventil angebracht, das einen zusätzlichen Gasballast einfließen lässt, um den Druck in der Kammer unabhängig von den anderen Gasflüssen regeln zu können. Mit diesem Ventil wird der Hintergrunddruck in der Vakuumkammer eingestellt. Dieser Druck ist notwendig um den Strahl der Lavaldüse einzufassen und gewährleistet so konstante Temperatur und Dichte im Überschallstrahl, wie in Abschnitt 3.2.2 genauer erläutert.

In Abbildung 3.6 ist der Kern der Apparatur, das Stagnationsvolumen dargestellt: Zwei Magnetventile V1, V2 (General Valve, series 9 und modifizierte BOSCH Einspritzdüse) befüllen das Stagnationsvolumen vor der Düse mit zwei unterschiedlichen Gasgemischen, wobei die Hauptkomponente mit einem Anteil $\geq 98 \%$ stets 


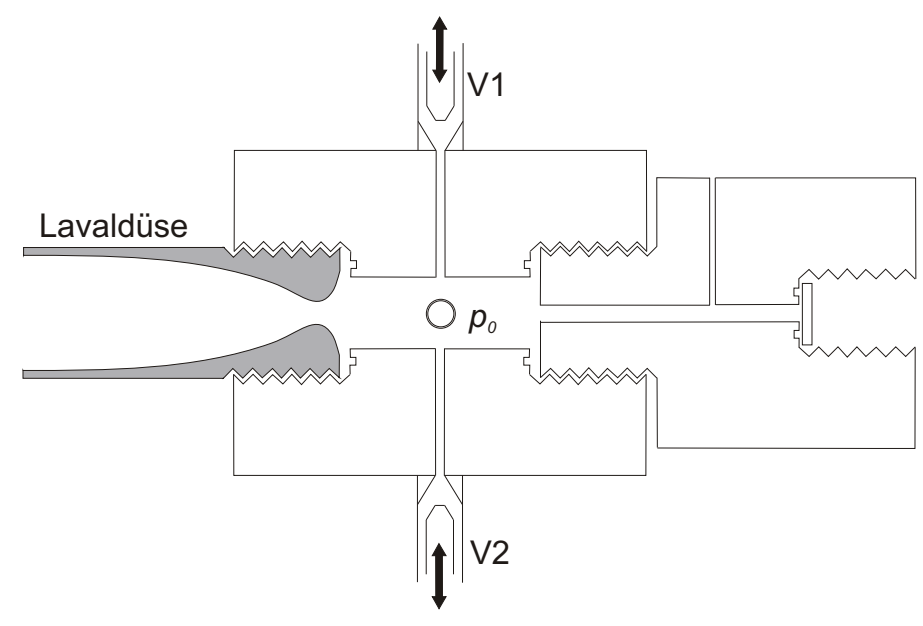

Abbildung 3.6: Das Stagnationsvolumen mit den Magnetventilen V1, V2, dem Drucksensor für $p_{0}$ und der Spülung für das Fenster, rechts im Bild

Stickstoff ist. Der Zufluss über das BOSCH-Ventil wird durch eine Waschflasche mit flüssigem $\mathrm{H}_{2} \mathrm{O}_{2}$ (Konz. $\geq 95 \%$, bei Experimenten mit Wasser etwa 63\%) geleitet und stellt so den Radikalvorläufer und Stickstoff für die Expansion bereit. Das andere Ventil (General Valve) führt ein Gemisch von Stickstoff und dem Reaktionspartner zu, das aus einer Edelgas-Vorratsflasche (Air-Liquide) entnommen wird. Zusätzlich wird ein kontinuierlicher Stickstoffstrom über eine Drossel am Fenster eingelassen, der das Fenster von allen anderen Stoffen der Expansion freihält, damit keine photochemischen Produkte seine optische Durchlässigkeit verschlechtern. Masseflussregler (Tylan FC260) ermöglichen die genaue Einstellung aller Gaszuflüsse, die Konzentration des Reaktionspartners in der Expansion ist somit als Parameter einzustellen. Die Durchflussregler werden für jede Gasmischung kalibriert, indem die Geschwindigkeit eines Druckanstiegs in einem bekannten Volumen gemessen wird. Die Zuflüsse über die Magnetventile sind darüber hinaus mit kapazitiven Drucksensoren (Baratron) ausgestattet, mit deren Hilfe die optimale Arbeit der Ventile überwacht wird. Die Taktrate der Ventile, sowie des gesamten Experiments beträgt $10 \mathrm{~Hz}$. Die Öffnungszeit der beiden Ventile wird überlappt und beträgt etwa 7 ms. Damit ist die chemische Kinetik, die bis zu einer maximalen Verzögerungs- 
zeit von $100 \mu$ s über den Fluoreszenznachweis von $\mathrm{OH}$ verfolgt wird, gegenüber der Düsenöffnungszeit sehr kurz.

Zur Messung von Raumtemperaturdaten wird die Lavaldüse durch ein Strömungsrohr ersetzt, das ein Fenster in Richtung des Sekundärelektronenvervielfachers besitzt. Zusätzlich wird die Pumpleistung gedrosselt um einen Druck von etwa 10 mbar in der Kammer zu erreichen. Die beweglichen Teile der Magnetventile werden entfernt und der Gasstrom so kontinuierlich in die Vakuumkammer eingelassen. Über den Druck im Strömungsrohr und das Verhältnis der Massendurchflüsse wird die Konzentration des Reaktionspartners bestimmt.

\subsection{1 Änderungen bei Messungen von KCSF}

Wie im Kapitel 2 eingeführt, wurden nicht nur kinetische Messungen von Reaktionen mit dem OH-Radikal untersucht, sondern auch Experimente zur Stoßdesaktivierung von hoch schwingungsangeregtem Toluol vorgenommen. Dazu wurde die Methode der KCSF, „,kinetically controlled selective fluorescence“, verwendet.

Das Prinzip des Pump-Probe Experiments bleibt unverändert, genau wie die Detektionsmethode der Fluoreszenz. Allerdings ändern sich durch die verwendeten Substanzen Cycloheptatrien (im folgenden CHT, als Vorläufer) und Toluol die Wellenlängen der Fluoreszenzanregung und der -detektion. Zur Erzeugung des hoch schwingungsangeregten Toluols wird CHT mit der Strahlung des Excimerlasers photolysiert. Dabei wird CHT zunächst in den $S_{1}$ Zustand angeregt, reagiert unter innerer Konversion zum hochangeregten $S_{0}$ Zustand und isomerisiert dann sehr schnell gegenüber der Stoßdesaktivierung zu hoch schwingungsangeregtem Toluol. Durch den Laserpuls des Excimerlasers wird also eine Population von Toluolmolekülen mit einer Energieverteilung erzeugt, die der thermischen Verteilung vor dem Puls entspricht, die dann um Photonenenergie und die Isomerisierungsenergie nach oben verschoben ist. Zur Detektion dieser Population wird die Fluoreszenz von Toluol mit Hilfe des Nd:YAG (355 nm, $70 \mathrm{~mJ})$ gepumpten Farbstofflasers mit Coumarin 152 in Ethanol mit Wellenlängen zwischen $266.8 \mathrm{~nm}$ und $267.5 \mathrm{~nm}$ angeregt. Als optische Filter werden ein hochreflektierender dielektrischer Spiegel (HR 225-240 nm $/ 45^{\circ}$ ), ein Interferenzfilter $\left(\lambda_{0}=277 \mathrm{~nm}, \mathrm{FWHM}=14 \mathrm{~nm}, T_{\max }=25 \%\right)$ und ein UV 
Kurzpassfilter (UG11) verwendet. Wie die Reaktionspartner des OH-Radikals wird CHT aus einer Edelstahlflasche (vorgemischt im jeweiligen Badgas) entnommen. Die Konzentration von CHT wird konstant unter einem Anteil von $\leq 1.6 \cdot 10^{-3}$ gehalten. Jeder Zeitpunkt dieser Experimente setzt sich aus der Überlagerung mehrerer $(5-8)$ experimenteller Zeitspuren zusammen, die ihrerseits eine Mittelung von 50 - 100 Einzelschuss pro Zeitpunkt enthalten. Die Extraktion der Ergebnisse für die mittlere pro Stoß übertragene Energie $\langle\langle E\rangle\rangle$ aus den so gewonnenen Primärsignalen ist im Abschnitt 2.3 beschrieben.

\subsection{Datenverarbeitung der Primärsignale bei Reaktionen des $\mathrm{OH}$-Radikals und zeitliche Steuerung des Experiments}

Die zeitabhängigen Signale der OH-Fluoreszenz lassen sich durch folgende (Photo-) chemischen Reaktionen erklären.

$$
\begin{aligned}
& \mathrm{H}_{2} \mathrm{O}_{2}+h \nu \rightarrow 2 \mathrm{OH}^{*} \\
& \mathrm{OH}^{*}+\mathrm{M} \stackrel{k_{\text {relax }}^{\prime}}{\longrightarrow} \mathrm{OH}+\mathrm{M} \\
& \mathrm{OH}+\mathrm{X} \stackrel{k_{\text {reakt }}^{\prime}}{\longrightarrow} \text { Produkte }
\end{aligned}
$$

wobei X einen der Reaktionspartner des OH-Radikals und M ein Badgasmolekül bezeichnen. Aus diesem vereinfachten Reaktionsschema folgt das Geschwindigkeitsgesetz für die detektierte Spezies $\mathrm{OH}$ :

$$
\frac{\mathrm{d}[\mathrm{OH}]}{\mathrm{d} t}=k_{\text {relax }}^{\prime}[\mathrm{OH}]^{*}[\mathrm{M}]-k_{\text {reakt }}^{\prime}[\mathrm{OH}][\mathrm{X}]
$$


Da die Badgaskonzentration $[\mathrm{M}]$ und die Konzentration des Reaktionspartners in Überschuss [X] als zeitlich konstant angenommen werden können, ergibt sich für die Konzentration von $\mathrm{OH}$ ein Verlauf pseudo-erster Ordnung.

$$
\frac{\mathrm{d}[\mathrm{OH}]}{\mathrm{d} t}=k_{\text {relax }}[\mathrm{OH}]^{*}-k_{\text {reakt }}[\mathrm{OH}]
$$

Das integrierte Geschwindigkeitsgesetz für diesen Fall lautet

$$
[\mathrm{OH}](t)=\frac{k_{\text {relax }}[\mathrm{OH}]_{0}}{k_{\text {reakt }}-k_{\text {relax }}} \cdot\left[\exp \left(-k_{\text {relax }} t\right)-\exp \left(-k_{\text {reakt }} t\right)\right]
$$

Die Konzentration der OH-Radikale beträgt bei Raumtemperatur etwa $10^{12} \mathrm{~cm}^{-3}$, im Strahl der verschiedenen Lavaldüsen $10^{11} \mathrm{~cm}^{-3}$. Der Anteil an schwingungsangeregten $\mathrm{OH}-$ Radikalen bei der Photolyse von $\mathrm{H}_{2} \mathrm{O}_{2}$ mit $193 \mathrm{~nm}$ beträgt etwa $2 \%$. Ein typischer Signalverlauf nach der Gleichung 3.26 ist in Abbildung 3.7 dargestellt. Im Kapitel 4 finden sich zur Beurteilung der Primärsignale für die verschiedenen Reaktionen analoge Abbildungen.

Das Intensitäts-Zeitprofil dieser Kinetik pseudo-erster Ordnung wird für verschiedene Konzentrationen des Reaktionspartners aufgenommen. Durch eine nichtlineare Anpassung von Gleichung 3.26 an die experimentellen Daten mit dem LevenbergMarquard-Algorithmus des Programms Origin (Microcal) wird die Geschwindigkeitskonstante pseudo-erster Ordnung $k_{\text {reakt }}$ bestimmt und gegen die Konzentration des Reaktionspartners im Überschuss aufgetragen. Diese ist aus dem Durchfluss durch die Massedurchflussregler und der Gesamtdichte im Gasstrahl aus den PitotRohr Messungen zu berechnen.

Aus der Steigung der resultierenden Geraden (siehe Abbildung 3.8) wird die Geschwindigkeitskonstante der Reaktion abgelesen. Durch die Messung in verschiedenen Lavaldüsen wird die Geschwindigkeitskonstante bei Temperaturen im Bereich 60 - $130 \mathrm{~K}$ und bei $298 \mathrm{~K}$ bestimmt. Diese Daten machen eine Aussage über die Temperaturabhängigkeit der Reaktion. Die so erhaltenen experimentellen Ergebnisse werden im Kapitel 4 für die einzelnen Reaktionen besprochen. Im Abschnitt 


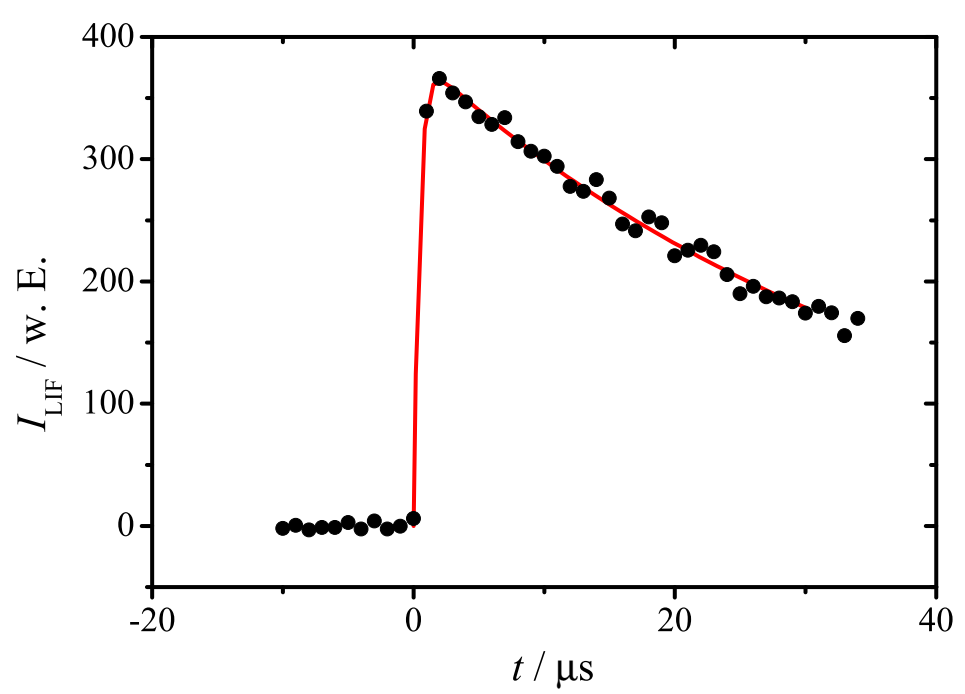

Abbildung 3.7: Ein typischer Signalverlauf der LIF-Intensität ist für eine Temperatur von $72 \mathrm{~K}$, Dichte von $0.95 \cdot 10^{17} \mathrm{~cm}^{-3}$ und eine Konzentration von $2.44 \cdot 10^{13} \mathrm{~cm}^{-3}$ an $N, N$-Dimethylformamid dargestellt.

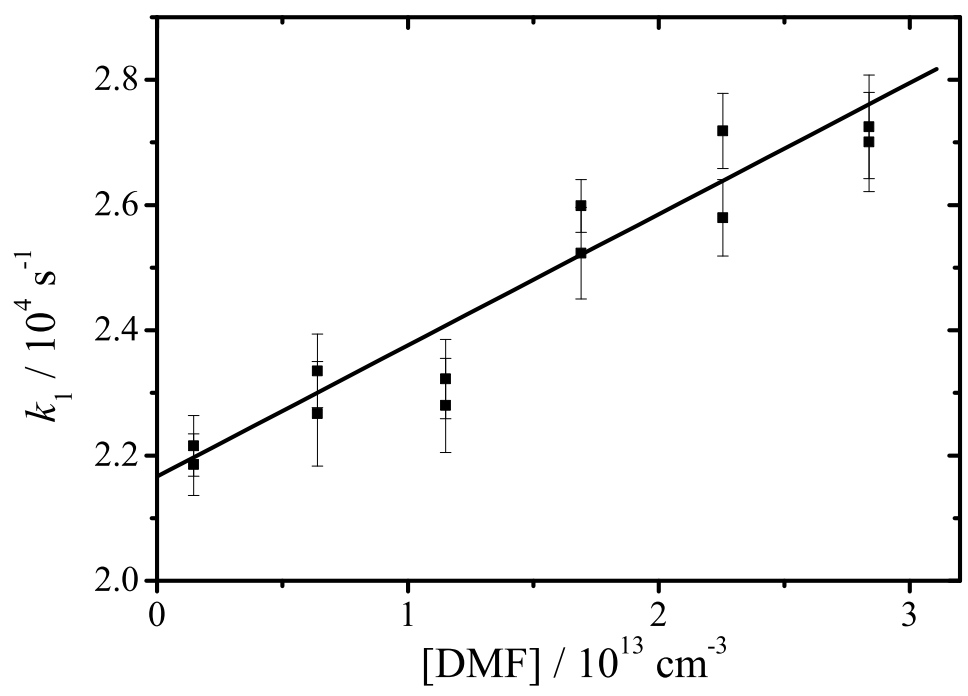

Abbildung 3.8: Die Geschwindigkeitskonstanten pseudo-erster Ordnung werden gegen die Konzentration von N,N-Dimethylformamid aufgetragen.Hier dargestellt für eine Temperatur von $72 \mathrm{~K}$ und eine Gesamtdichte von $0.95 \cdot 10^{17} \mathrm{~cm}^{-3}$. Die Geradensteigung ergibt bei dieser Einzelmessung eine Geschwindigkeitskonstante von $(2.0 \pm 0.1)$. $10^{-10} \mathrm{~cm}^{3} \mathrm{~s}^{-1}$. 
2.3 findet sich die Auswertmethode und im Abschnitt 4.1 die Ergebnisse der Messungen zum Schwingungsenergietransfer von Toluol.

Die zeitliche Abfolge der Einzelexperimente, die aus dem Öffnen der Magnetventile, dem Feuern der Laser und der Datenaufnahme bestehen, wurde von zwei DG535-Stanford Research Delay-Generatoren koordiniert. Diese wurden über eine GPIB-Verbindung vom PC gesteuert. Während der Experimente zu dieser Arbeit wurde das Messprogramm und der steuernde PC erneuert. Aktuell wird ein HPVEEbasiertes Messprogramm zur Steuerung der Experimente und zur Datenaufnahme verwendet. Der Grund für die Aktualisierung liegt in der Integration des neuen PC's in das LAN der Abteilung und der Aktualisierung der verwendeten PCI-Karten.

\subsection{Verwendete Substanzen}

Als Radikalvorläufer wurde Wasserstoffperoxid eingesetzt, das von der Firma Solvay Interox mit 70 Gew. \% Reinheit bezogen wurde. Zur Entfernung des enthaltenen Wassers wurde diese Mischung in eine Waschflasche gefüllt und über Nacht Stickstoff hindurchgeleitet um eine Konzentration $\geq 95 \%$ herzustellen. Für Messungen in Gegenwart von Wasserdampf wurde eine 63 Gew. \% konzentrierte wässrige Mischung von Wasserstoffperoxid verwendet. Die Normalsiedepunkte $\mathrm{T}_{\mathrm{S}}$ der Reaktionspartner von $\mathrm{OH}$ sind angegeben, um eine vergleichende Abschätzung der Aggregationsneigung vornehmen zu können. Gase wurden von Air Liquide bezogen: Stickstoff $99.996 \%$, Acetylen $99.6 \%\left(\mathrm{~T}_{\mathrm{S}}=189 \mathrm{~K}\right)$, Ethen $99.5 \%\left(\mathrm{~T}_{\mathrm{S}}=169 \mathrm{~K}\right)$, Kohlendioxid $99.995 \%$, Helium 99.996\%, Krypton $99.996 \%$ und Argon 99.998\%. Von Fluka wurden bezogen: Benzol $\geq 99 \%\left(\mathrm{~T}_{\mathrm{S}}=353 \mathrm{~K}\right)$, N,N-Dimethylformamid $\geq 99 \%$ $\left(\mathrm{T}_{\mathrm{S}}=426 \mathrm{~K}\right)$, Acetaldehyd $\geq 95 \%\left(\mathrm{~T}_{\mathrm{S}}=294 \mathrm{~K}\right)$. L-Alaninethylester Hydrochlorid $\geq 99 \%$ wurde von Alfa Aesar bezogen. Der Ester wurde durch Ausschütteln mit einer wässrigen Lösung von Kaliumcarbonat aus dem Hydrochlorid freigestetzt und mit Dichlormethan extrahiert, über Magnesiumsulfat getrocknet und das Lösungsmittel im Unterdruck entfernt. Eine genaue Arbeitsvorschrift ist in Referenz [107] zu finden. Alle anderen Substanzen wurden ohne weitere Reinigung verwendet. 



\section{Kapitel 4}

\section{Ergebnisse und Diskussion}

Wegen der relativ unterschiedlichen Ergebnisse, die in diesem Kapitel vorgestellt werden, bietet sich eine thematische Gliederung an. Die einzelnen Abschnitte beinhalten aus Gründen der Übersichtlichkeit sowohl die Ergebnisse als auch ihre Diskussion. Beginnend mit den Ergebnissen zum Schwingungsenergietransfer werden danach die Ergebnisse zur Kinetik des OH-Radikals vorgestellt.

\subsection{Schwingungsrelaxation von Toluol}

Die im Abschnitt 2.3 dargestellte Methode der KCSF („kinetically controlled selective fluorescence") von Toluol ist zur Untersuchung des Schwingungsenergietransfers zwischen hoch schwingungsangeregtem Toluol und verschiedenen Stoßgasen verwendet worden. Dabei wurden die Messungen für jedes Gas bei Raumtemperatur und bei einer tiefen Temperatur unter $100 \mathrm{~K}$ durchgeführt. Zunächst sollen die Primärsignale für die untersuchten Badgase Stickstoff, Helium und Kohlendioxid dargestellt, und eine einfache analytische Auswertung vorgenommen werden. Anschließend werden die Ergebnisse mit Werten für $\langle\langle\Delta E\rangle\rangle$ aus der Literatur verglichen und diskutiert.

Die Ergebnisse für die kürzeste Probe-Wellenlänge von $266.8 \mathrm{~nm}$ sind in Abbildung 4.1 zusammengefasst. Die Signale zeigen die Fluoreszenzausbeute der Toluolmoleküle in Abhängigkeit von der Anzahl der Stöße, die die Population nach der Anregung erfahren hat. Sie weisen einen sigmoiden Verlauf auf, da das Beobachtungsfenster in den thermisch equilibrierten Bereich hineinreicht und die größte 
Intensität der Fluoreszenz für die vollständig relaxierte, thermische Verteilung der Toluolmoleküle erreicht wird. Die Anfangsenergie der Toluolmoleküle betrug dabei $52100 \mathrm{~cm}^{-1}$ und die obere Grenze des Beobachtungsfensters wurde auf $1500 \mathrm{~cm}^{-1} \mathrm{ab}-$ geschätzt. Die Photolyse von Cycloheptatrien mit einem Laserpuls der Wellenlänge $\lambda=248 \mathrm{~nm}$ erzeugte dabei die schwingungsheiße Toluolpopulation, deren Desaktivierung durch die Fluoreszenz beobachtet wurde. Wie in Abschnitt 2.3 eingehender betrachtet, führen strahlungslose Konkurrenzprozesse (vornehmlich IC) zu einer starken Abhängigkeit der Fluoreszenzausbeute von der Schwingungsanregung im $\mathrm{S}_{1}$-Zustand der Toluolmoleküle aus dem die Fluoreszenz nach der Anregung durch den Probe-Laserpuls erfolgt. Die Signale sind auf das Maximum normiert, auf der Ordinate ist die Anzahl der Lennard-Jones-Stöße aufgetragen, die die Toluolpopulation erfahren hat. Die Parameter zur Berechnung der Stoßzahl sind Referenz [101] entnommen. Die Signale, die bei Zimmertemperatur ermittelt wurden, weisen einen späteren Anstieg auf als die Signale der Messung im Strahl der verwendeten Lavaldüsen, unabhängig vom Stoßpartner. Nach der analytischen Auswertmethode von Reihs et al. [100] ergeben sich für die mittlere pro Stoß übertragene Energie im Energieintervall zwischen $52100 \mathrm{~cm}^{-1}$ und $1500 \mathrm{~cm}^{-1}$ folgende Werte:

$\begin{array}{lccccc}\text { Badgas } & T / \mathrm{K} & \langle\langle\Delta E\rangle\rangle / \mathrm{cm}^{-1} & \text { Anzahl Messungen } & \text { CHT-Anteil } & p / \mathrm{Pa} \\ \mathrm{He} & 298 & -50 & 8 & 4.1 \cdot 10^{-4} & 1218 \pm 10 \\ & 65 & -73 & 5 & 4.1 \cdot 10^{-4} & 42 \pm 10 \\ \mathrm{~N}_{2} & 298 & -100 & 5 & 4.1 \cdot 10^{-4} & 903 \pm 5 \\ & 88 & -136 & 5 & 1.6 \cdot 10^{-3} & 137 \pm 7 \\ \mathrm{CO}_{2} & 298 & -179 & 5 & 4.1 \cdot 10^{-4} & 536 \pm 5 \\ & 75 & -287 & 5 & 4.1 \cdot 10^{-4} & 99 \pm 7\end{array}$

Für Stickstoff und Helium stehen weitere Tieftemperaturdaten von der Arbeitsgruppe um I. W. Smith [55] zum Vergleich zur Verfügung. Für die Raumtemperaturdaten gibt es eine ganze Reihe von Daten zur mittleren pro Stoß übertragenen Energie, die mit unterschiedlichen Methoden ermittelt wurden. 

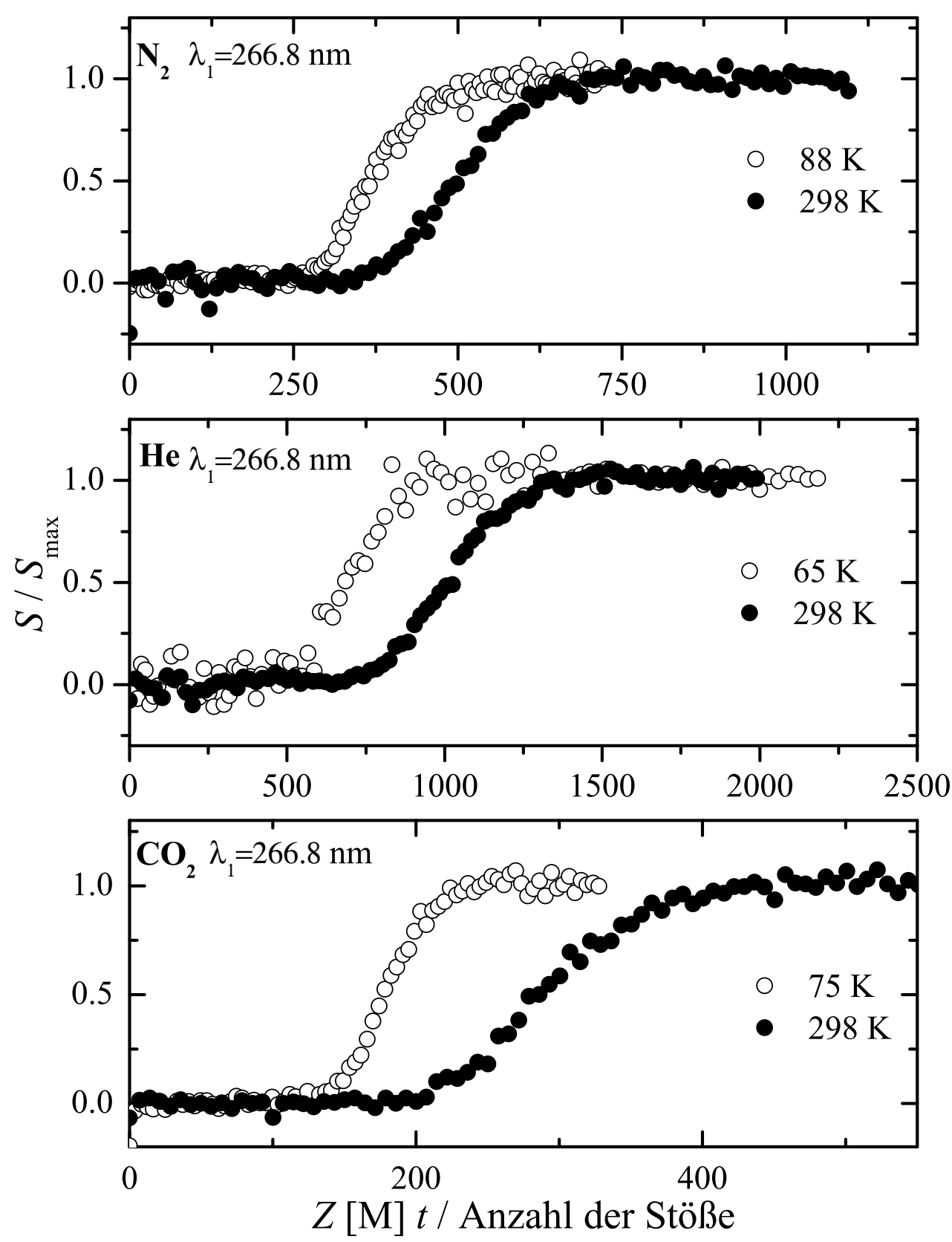

Abbildung 4.1: Die verschiedenen Signale der KCSF für die Stoßgase Stickstoff, Helium und Kohlendioxid bei einer Probe-Wellenlänge von $266.8 \mathrm{~nm}$ 

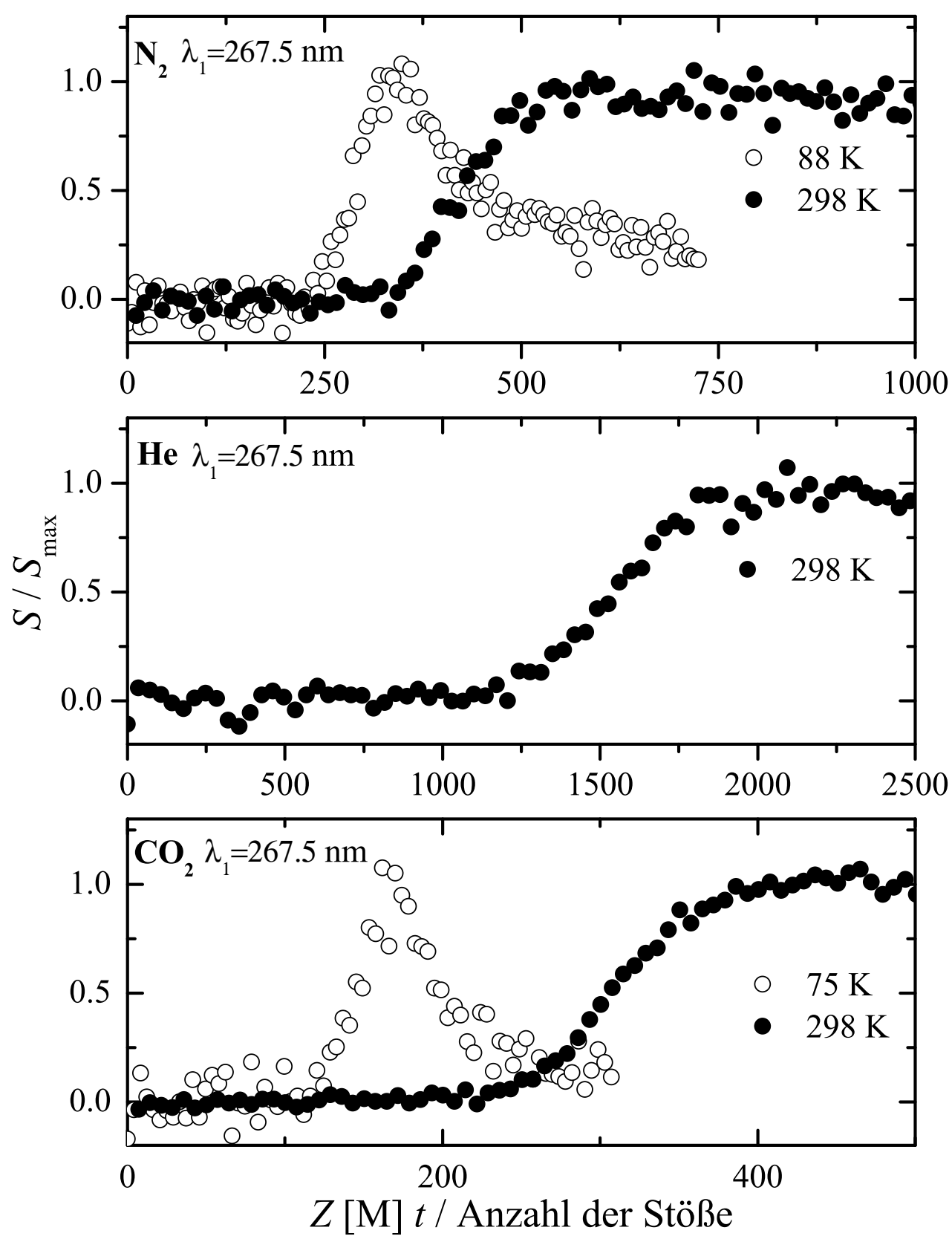

Abbildung 4.2: Die verschiedenen Signale der KCSF für die Stoßgase Stickstoff, Helium und Kohlendioxid bei einer Probe-Wellenlänge von $267.5 \mathrm{~nm}$ 
Die Tieftemperaturmessung verwendete die Infrarot-Fluoreszenz der Toluolmoleküle um den Energietransfer zu Helium, Stickstoff und Argon zu untersuchen. Dazu wurde von Wright et al. die IR-Emission der C-H-Streckschwingungen von Toluol zeitaufgelöst detektiert. Die Werte von $\langle\langle\Delta E\rangle\rangle$ betragen für Stickstoff und Helium bei Raumtemperatur $-147 \mathrm{~cm}^{-1}$ und $-70 \mathrm{~cm}^{-1}$ bei einer Anregungsenergie von $44000 \mathrm{~cm}^{-1}$ [55]. Die Werte zeigen in diesen Experimenten keine Temperaturabhängigkeit bei Stickstoff und eine leichte Abnahme mit der Temperatur für Helium. In diesem Punkt besteht also ein Widerspruch zu den hier vorgelegten Daten.

Für Raumtemperatur werden die Daten mit den Ergebnissen von Hippler et al. [101], Lenzer et al. [62] und Tosselli et al. [108] verglichen. Die UV-Absorptionsexperimente von Hippler et al.nutzten die lineare Abhängigkeit des Absorptionsquerschnitts von Toluol bei $\lambda=223 \mathrm{~nm}$ von der inneren Energie zur Ermittlung von $\langle\langle\Delta E\rangle\rangle$. Die Werte stellen eine Mittelung über den Energiebereich zwischen $52100 \mathrm{~cm}^{-1}$ und $4100 \mathrm{~cm}^{-1}$ dar. Die Werte betragen $-75 \mathrm{~cm}^{-1},-130 \mathrm{~cm}^{-1}$ und $-280 \mathrm{~cm}^{-1}$ für Helium, Stickstoff und Kohlendioxid.

Die KCSI-Methode liefert nicht nur Werte für die mittlere pro Stoß übertragene Energie in Abhängigkeit von der inneren Energie $\langle\Delta E(E)\rangle$ sondern auch die Form der Verteilungsfunktion $P\left(E^{\prime}, E\right)$ wenn eine hinreichende Anzahl von Datensätzen verschiedener Stoßpartner und Probe-Wellenlängen zur Verfügung steht. Bei einer inneren Energie von $10000 \mathrm{~cm}^{-1}$ ergeben sich $-37 \mathrm{~cm}^{-1}$ und $-134 \mathrm{~cm}^{-1}$ für die Desaktivierung durch Helium und $\mathrm{CO}_{2}$, bei $20000 \mathrm{~cm}^{-1}$ betragen die Werte $-75 \mathrm{~cm}^{-1}$ und $-277 \mathrm{~cm}^{-1}$.

Eine weitere Arbeit, die den Prozess mit zeitaufgelöster Infrarot-Fluoreszenz untersuchte, ist von Tosselli et al. veröffentlicht worden. Dort finden sich für eine Energie von $24000 \mathrm{~cm}^{-1}$ für $\langle\langle\Delta E\rangle\rangle$ von Helium $-62 \mathrm{~cm}^{-1}$, von Stickstoff $-139 \mathrm{~cm}^{-1}$ und von $\mathrm{CO}_{2}-245 \mathrm{~cm}^{-1}$

Grundsätzlich findet sich eine zufriedenstellende Übereinstimmung zwischen den Daten dieser Arbeit und denen anderer Experimente, besonders im Hinblick auf die unterschiedlichen experimentellen Methoden, die zum Einsatz kamen. Die Werte dieser Arbeit sind kleiner als der Mittelwert der verschiedenen Vergleichsdaten.

Der Einsatz einer optischen Filterkombination, der wegen der Streustrahlung des Photolyselasers nötig war, hat eine zusätzliche Selektivität für die Beobachtung 
der schwingungsheißen Moleküle erzeugt. Die Beobachtungsfenster sind dadurch gegenüber der KCSI verändert, was dazu beitragen kann, die Unterschiede der Signale der KCSI und den hier vorgestellten Daten zu erklären. Die Ergebnisse von Frerichs et al. [61] haben gezeigt, dass die Beobachtungsfenster der KCSF ohne zusätzliche spektrale Diskriminierung der Fluoreszenz mit denen der KCSI übereinstimmen. Die detaillierten Untersuchungen der energieabhängigen $\langle\Delta E(E)\rangle$ haben eine lineare Abhängigkeit von der Energie der Toluolmoleküle ergeben, allerdings geht diese Größe bei Energien unter $4000 \mathrm{~cm}^{-1}$ durch die Bedingung der mikroskopischen Reversiblität schnell gegen Null. Die obere Grenze des Beobachtungsfensters, das zur Auswertung angenommen wurde, liegt in diesem Bereich. Bei tieferer Temperatur sollte sich der Energiebereich, in dem die mikroskopische Reversiblität relevant ist, zu kleineren Energien verschieben. Auch wenn die Größe dieses Effekts nicht genau bekannt ist, würde er niedrige Werte bei Raumtemperatur und einen Anstieg zu tiefen Temperaturen qualitativ erklären. Die Tieftemperaturdaten dieser Arbeit von $\langle\langle\Delta E\rangle\rangle$ stimmen auffallend mit den Werten anderer Arbeiten für Raumtemperatur überein.

Die in Abbildung 4.2 dargestellten Signale der längeren Probe-Wellenlängen werden qualitativ behandelt. Die längere Probe-Wellenlänge verschiebt die Grenzen des Beobachtungsfensters zu höheren Energien. Die untere Grenze des Beobachtungsfensters ist nicht mehr mit dem Schwingungsgrundzustand des Toluols identisch. Bei den tiefen Temperaturen fallen die Signale nach dem Durchlaufen eines Maximums auf einen konstanten Wert ab, der der thermischen Besetzung entspricht. Die Grenzen des Beobachtungsfensters für diese Wellenlänge lassen sich weniger gut abschätzen als für die Repopulierung des Grundzustands. Die Temperaturabhängigkeit des Signal-Endniveaus könnte bei einer Reihe temperaturabhängiger Messungen allerdings die untere Grenze des Beobachtungsfensters festlegen.

Abschließend sollen mögliche systematische Fehler der angewandten Methode betrachtet werden. Die Photolyse von Cycloheptatrien erzeugt ein hoch schwingungsangeregtes Ensemble von Toluolmolekülen, neben der Desaktivierung durch Stöße mit dem Badgas besteht die Möglichkeit der unimolekularen Reaktion zum Benzylradikal und einem Wasserstoffatom. Die unimolekulare Reaktion besitzt eine ausgeprägte Energieabhängigkeit, bei einer inneren Energie von $52000 \mathrm{~cm}^{-1}$ beträgt 
die Geschwindigkeitskonstante $1.5 \cdot 10^{-6} \mathrm{~s}^{-1}$ [63]. Schon nach wenigen Stößen spielt dieser Konkurrenzprozess keine Rolle mehr. Der Einfluss von Stößen der Toluolmoleküle mit CHT kann vernachlässigt werden, bei den verwendeten Stoffmengenanteilen unter $3 \cdot 10^{-3}$ wird in allen Experimenten weniger als $1 \%$ der Schwingungsenergie der Toluolmoleküle durch CHT aufgenommen, wenn CHT sechs mal effektiver als Stickstoff bezüglich des Energietransfers angenommen wird.

Bei den tiefen Temperaturen könnte die Bildung von CHT-Dimeren eine Rolle für den beobachteten Energietransfer spielen. Allerdings lässt sich durch einen Vergleich mit der Dimerbildungskonstante von Benzol, die von Hamon et al. [17] mit der CRESU-Technik in einem weiten Temperaturbereich untersucht wurde, für die gewählten experimentellen Bedingungen die Bildung einer größeren Menge an Dimeren ausschließen.

Die Experimente haben gezeigt, dass die KCSF-Methode mit der Laval-Apparatur kombinierbar ist. Der Einfluss der optischen Filter vor dem Detektor auf die Ergebnisse sollte überprüft werden, indem eine Photolysequelle verwendet wird, die den Verzicht auf diese Filter ermöglicht. Mit einer Erweiterung des Datensatzes wären dann auch weitere Aussagen zur Temperaturabhängigkeit des Schwingungsenergietransfers möglich.

\subsection{Zusammenfassung der Reaktionen von $\mathrm{OH}$ mit ungesättigten Kohlenwassertoffen}

Die Diplomarbeit von Rosalin Karunanandan [109] und die Dissertation von Tim Spangenberg [34] beschäftigten sich mit der Reaktion zwischen Acetylen, Ethen und Benzol und dem OH-Radikal. Mit Ausnahme der Reaktion von Acetylen zeigen diese Reaktionen eine negative Temperaturabhängigkeit. Bei allen handelt sich um Additionsreaktionen, im Gegensatz zu den Abstraktionsreaktionen, die andere Abschnitte dieses Kapitels behandeln. Daher wird auch eine Betrachtung der Druckabhängigkeit der Reaktionen durchgeführt. Hier soll lediglich ein kurzer Rückblick auf diese abgeschlossene Serie untersuchter Reaktionen vorgenommen werden, wie er in Referenz [28] vor kurzem publiziert wurde. 
Den Reaktionen scheint neben dem Additionsmechanismus auch die Ausbildung eines nicht-kovalent gebundenen Komplexes (oft als $\pi$-Komplex bezeichnet) vor der Ausbildung einer kovalenten Bindung gemeinsam zu sein. Für Acetylen und OH konnte ein solcher Komplex spektroskopisch nachgewiesen werden [110]. Das Wasserstoffatom des Radikals ist in diesem Komplex auf die Bindungsmitte der Acetylen C-C Dreifachbindung gerichtet, so dass der Komplex eine T-förmige Geometrie hat. Zur Addition des OH-Radikals muss sich das Radikal drehen. Für andere Kohlenwasserstoffe sind ähnliche Strukturen rechnerisch vorhergesagt worden [111, 112]. Zwischen dem $\pi$-Komplex und dem kovalent gebundenen Addukt ( $\sigma$-Komplex) bildet sich eine Barriere aus, die verschiedene Ursachen haben kann: Die Barriere kann auf eine vermiedenen Kreuzung zwischen zwei elektronischen Potentialflächen, die Drehung des OH-Radikals oder die Umhybridisierung des Kohlenstoffatoms zurückgeführt werden [113]. Die Höhe dieser Barriere relativ zu den isolierten Edukten entscheidet über die Temperaturabhängigkeit einer solchen Reaktion. Liegt die Barriere höher oder sehr nahe an der Energie der Edukte, so zeigt die Reaktion eine positive Temperaturabhängigkeit oder ist nahezu temperaturunabhängig, wie im Fall von Acetylen und $\mathrm{OH}$ (vgl. Abb. 4.3). Die Überwindung dieser Barriere ist der geschwindigkeitsbestimmende Schritt. Für die Reaktion zwischen Acetylen und $\mathrm{OH}$ sind Aktivierungsenergien von $1.4 \mathrm{kcal} / \mathrm{mol}$ [114] und $1.8 \mathrm{kcal} / \mathrm{mol}$ [115] experimentell bestimmt worden. Theoretische Berechnungen geben die Aktivierungsenergie mit $1.6 \mathrm{kcal} / \mathrm{mol}$ [116] und $1.4 \mathrm{kcal} / \mathrm{mol}$ [92] an.

Bei dieser Reaktion handelt es sich um eine Additionsreaktion, bei der ein energetisch nicht equilibiertes Produkt entsteht, das durch Stöße stabilisiert werden kann. Deshalb zeigt die Reaktion eine Druckabhängigkeit. Bei den Drücken, die in der Laval-Apparatur erreicht werden können, befindet sich diese Reaktion im Fall-Off Bereich. Die Werte der Fall-Off Geschwindigkeitskonstanten von Acetylen und OH sind in Abbildung 4.3 weiß dargestellt. Neben der Messung bei hinreichend hohen Drücken, wie bei den Daten von Fulle et al. geschehen, gibt es eine alternative Möglichkeit die Hochdruck-Geschwindigkeitskonstante dieser Reaktion zu bestimmen.

Im Hochdruckgrenzfall ist die Bildung des Adduktes der geschwindigkeitsbestimmende Schritt. Diese Situation wird bei der Schwingungsdesaktivierung analog wie- 


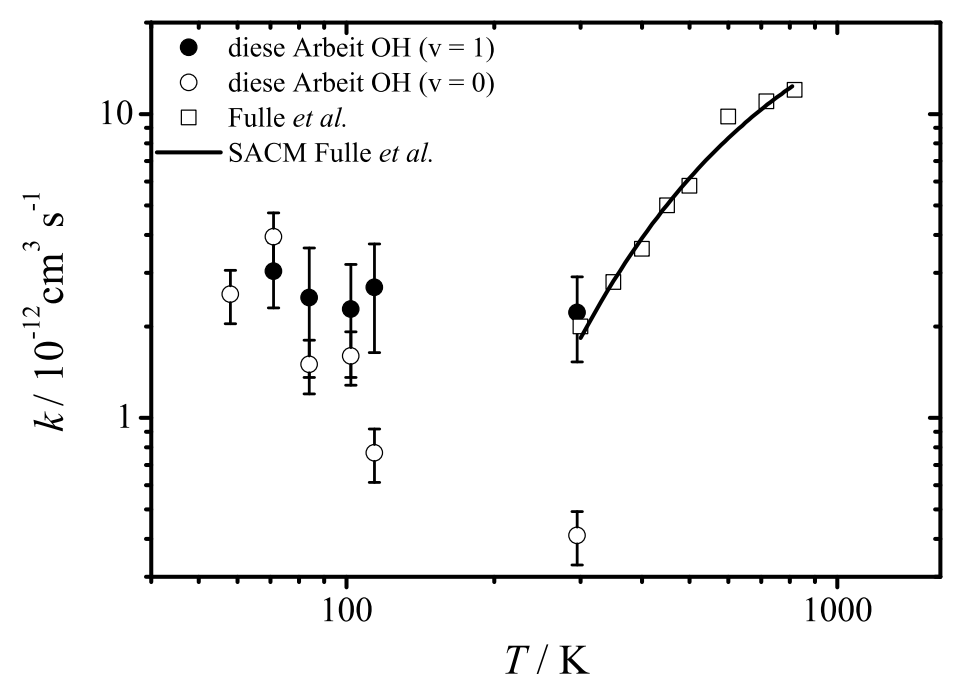

Abbildung 4.3: Die Hochdruck-Additionsgeschwindigkeitskonstante der Reaktion von Acetylen mit $\mathrm{OH}$ ist hier zusammen mit den experimentellen Daten und SACM-Berechnungen von Fulle et al. dargestellt [115]. Die in Göttingen ermittelten Geschwindigkeitskonstanten sind die Relaxationsgeschwindigkeiten von $\mathrm{OH}(\mathrm{v}=1)$ und die Geschwindigkeitskonstanten mit $\mathrm{OH}(\mathrm{v}=0)$.

$\begin{array}{lcccc}\text { Düse } & T / \mathrm{K} & \rho / 10^{17} \mathrm{~cm}^{-3} & k / 10^{-12} \mathrm{~cm}^{3} \mathrm{~s}^{-1} \\ & 298 & 3.8 & (0.41 \pm 0.08) & (2.22 \pm 0.69) \\ \text { - } & 114 & 1.0 & (0.77 \pm 0.15) & (2.69 \pm 1.05) \\ \text { M211E17 } & 14.52 & (1.60 \pm 0.32) & (2.28 \pm 0.92) \\ \text { M278E16 } & 101 & 0.52 & (1.50 \pm 0.30) & (2.49 \pm 1.13) \\ \text { M351E17 } & 84 & 0.97 & (3.91 \pm 0.79) & (3.04 \pm 0.74) \\ \text { M408E16 } & 71 & 0.90 & (2.55 \pm 0.51) & -\end{array}$

Tabelle 4.1: Mit Hilfe der Göttinger CRESU-Apparatur gemessene Geschwindigkeitskonstanten für die Reaktion von $\mathrm{OH}$ mit Acetylen: Die Geschwindigkeitskonstanten wurden mit mindestens drei Einzelmessungen ermittelt. Die maximale Konzentration von Acetylen war $\rho_{\text {Acetylen }}=$ $2 \cdot 10^{15} \mathrm{~cm}^{-3}$ 
dergegeben, auch hier ist die Geschwindigkeitskonstante durch die Bildung eines Stoßkomplexes gegeben. Die Relaxationsgeschwindigkeitskonstante von $\mathrm{OH}(\mathrm{v}=1)$, schwarz dargestellt, gibt die obere Grenze für den Hochdruckgrenzwert an. Die Übereinstimmung mit den Hochdruckdaten von Fulle et al. zeigt die Gültigkeit der oben angeführten Näherung für diese Reaktion. Die Werte der Geschwindigkeitskonstanten, die in Göttingen ermittelt wurden, sind in Tabelle 4.1 zusammengefasst.

Für die Reaktion von Ethen mit $\mathrm{OH}$ ist experimentell eine negative Temperaturabhängigkeit etabliert. Die theoretischen Berechnungen weichen teilweise voneinander ab, aber jüngere Studien kommen zu einer qualitativ übereinstimmenden Beschreibung. Danach bildet sich ein lockerer $\pi$-Komplex zwischen dem OH-Radikal und Ethen, der über einen Übergangszustand nahe der Energie der Edukte zum primären Addukt, dem $\sigma$-Komplex, reagiert. Für den $\pi$-Komplex ergeben sich zwei Zerfallskanäle: Der erste Kanal stellt die Umkehrreaktion zu seiner Bildung aus den Edukten $\mathrm{OH}$ und Ethen dar, der andere Kanal führt zum $\sigma$-Komplex. Die experimentell zu bestimmende Netto-Geschwindigkeitskonstante $k_{\exp }$ kann bei der Reaktion über den $\pi$-Komplex durch

$$
k_{\text {exp }}=k_{\text {cap }}\left[\frac{k_{\text {diss }, \mathrm{v}}}{k_{\text {diss }, \mathrm{v}}+k_{\text {diss }, \mathrm{r}}}\right]
$$

in Analogie zu Gleichung 2.24 ausgedrückt werden. Bei $k_{\text {cap }}$ handelt es sich um eine Einfanggeschwindigkeitskonstante, wie sie in Kapitel 2 entwickelt wurde. Diese Grenzgeschwindigkeit wird durch den folgenden Bruch verringert. Dabei ist $k_{\text {diss, } \mathrm{v}}$ die Geschwindigkeitskonstante zum $\sigma$-Komplex und $k_{\text {diss,r }}$ steht für die Redissozation zu den Edukten.

Alle Geschwindigkeitskonstanten sind als spezifische Geschwindigkeitskonstanten aufzufassen. Die Konkurrenz zwischen den Zerfallskanälen des Komplexes ist für die Temperaturabhängigkeit verantwortlich, wie im Abschnitt 2.2.5 dargestellt. Die Reaktion kann von bevorzugter Rückreaktion des $\pi$-Komplexes zu den Edukten bis zur bevorzugten Reaktion zum Addukt variieren, abhängig von der Energie- und Drehimpulsabhängigkeit der beiden Zerfallskanäle des $\pi$-Komplexes. Es findet also „rotational-“ und „vibrational channel switching“ statt. 
Singleton und Cvetanovic veröffentlichten als Erste die Vermutung, dass solche schwach gebundenen Komplexe eine Ursache für eine negative Temperaturabhängigkeit sein können [117]. Dieses System ist auf der Basis der hier vorgestellten Situation mit detaillierten Berechnungen untersucht worden [94, 93], wobei die Ergebnisse der Berechnung der Geschwindigkeitskonstante über große Temperaturbereiche im Einklang mit den verfügbaren experimentellen Daten stehen.

Die Lebensdauer des schwach gebundenen $\pi$-Komplexes ist so kurz, dass unter den meisten experimentellen Bedingungen keine Stabilisierung durch Stöße stattfinden kann. Die Druckabhängigkeit dieser Additionsreaktion wird durch die Stabilisierung des $\sigma$-Komplexes bestimmt.

Abbildung 4.4 zeigt die im Rahmen der Dissertation von Tim Spangenberg [34] ermittelten Tieftemperaturdaten der Reaktion zusammen mit den Hochdruckdaten höherer Temperaturen von Fulle et al. [115]. Für die Geschwindigkeitskonstante dieser Arbeit wurde die Relaxationsgeschwindigkeitskonstante schwingungsangeregter OH-Radikale ( $\mathrm{v}=1$ ) zur Bestimmung der Hochdruck-Rekombinations-Konstanten verwendet, wie bei der Reaktion von Acetylen und $\mathrm{OH}$ dargestellt. Die Fall-Off Konstanten mit $\mathrm{OH}$ im Schwingungsgrundzustand sind ebenfalls aufgetragen und in Tabelle 4.2 dargestellt.

Die Reaktion von Benzol mit $\mathrm{OH}$ ist mechanistisch komplizierter als die der bisher betrachteten Kohlenwasserstoffe. Der vergleichsweise niedrige Dampfdruck und die spektroskopischen Eigenschaften von Benzol stellten neue Anforderungen an die Durchführung der Experimente. Gleichzeitig macht die Größe des Moleküls eine Betrachtung der Schwingungsrelaxation des OH-Radikals unnötig, da diese Reaktion bei den verwendeten Drücken im Hochdruckbereich ist. Abbildung 4.5 zeigt die Geschwindigkeitskonstante der Reaktion mit den in Göttingen ermittelten Daten [109] und einigen Werten aus der Literatur: Das Minimum der Geschwindigkeitskonstante bei etwa $400 \mathrm{~K}$ wird einem Mechanismuswechsel von der H-Abstraktion zur OH-Addition zugeschrieben [118, 119]. Für die Additionsreaktion streuen die Werte der Aktivierungsenergie, die in bisher veröffentlichten Arbeiten genannt werden, um Null. Es ist noch nicht eindeutig erwiesen, ob sich eine absolute Barriere in der Reaktionskoordinate findet. Die Tieftemperaturdaten, die in der Diplomarbeit von Rosalin Karunanandan [109] vorgestellt wurden und hier kurz aufgegriffen wer- 


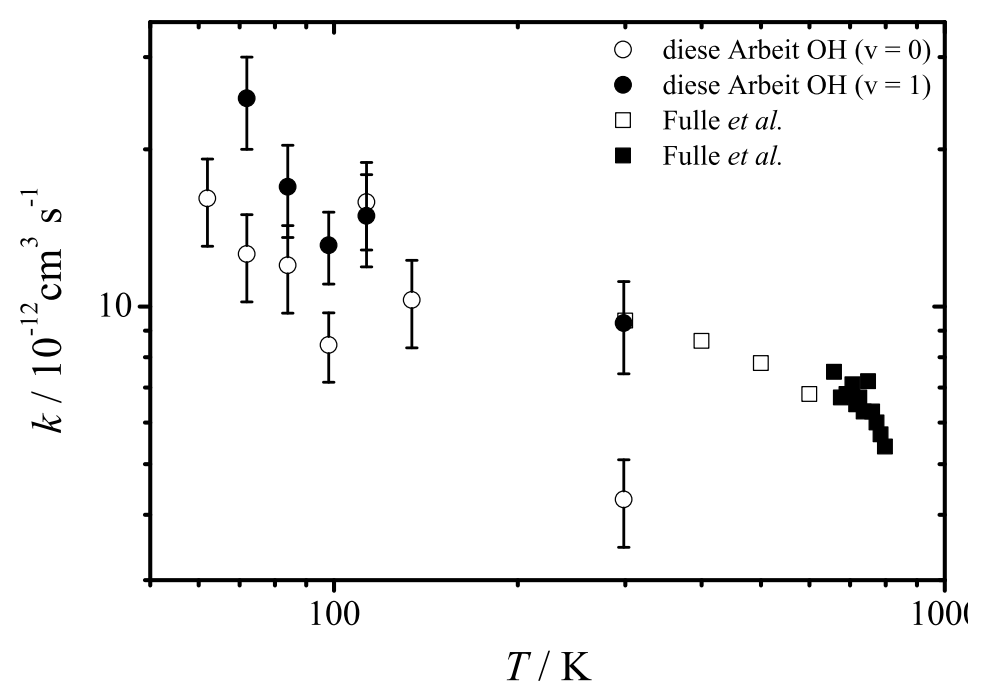

Abbildung 4.4: Die Geschwindigkeitskonstante der Reaktion von Ethen mit OH ist hier zusammen mit den experimentellen Daten von Fulle et al. dargestellt [115]. Die in Göttingen ermittelten Geschwindigkeitskonstanten sind die Relaxationsgeschwindigkeiten von $\mathrm{OH}(\mathrm{v}=1)$ und die Geschwindigkeitskonstanten mit $\mathrm{OH}(v=0)$.

$\begin{array}{lcccc}\text { Düse } & T / \mathrm{K} & \rho / 10^{17} \mathrm{~cm}^{-3} & k / 10^{-12} \mathrm{~cm}^{3} \mathrm{~s}^{-1} \\ & 298 & 2.7 & (4.2 \pm 0.8) & (9.3 \pm 1.9) \\ \text { - } & 134 & 1.3 & (10.3 \pm 2.0) & - \\ \text { M211E17 } & 113 & 1.0 & (15.9 \pm 3.0) & (14.9 \pm 3.0) \\ \text { M278E16 } & 98 & 0.55 & (8.5 \pm 1.3) & (13.1 \pm 2.1) \\ \text { M351E17 } & 84 & 0.97 & (12.0 \pm 2.2) & (10.7 \pm 3.4) \\ \text { M408E16 } & 72 & 0.93 & (12.6 \pm 2.4) & (25.0 \pm 5.2) \\ \text { M508E16 } & 62 & 0.60 & (16.1 \pm 3.1) & -\end{array}$

Tabelle 4.2: Geschwindigkeitskonstanten für die Reaktion von $\mathrm{OH}$ mit Ethen: Die Geschwindigkeitskonstanten wurden mit mindestens drei Einzelmessungen ermittelt. Die maximale Konzentration von Ethen war $\rho_{\text {Ethen }}=$ $2 \cdot 10^{15} \mathrm{~cm}^{-3}$ 
den sollen, zeigen eine ausgeprägt negative Temperaturabhängigkeit. Damit legen sie eine ähnliche Situation wie im Fall der Reaktion von Ethen und $\mathrm{OH}$ nahe, wo keine ausgeprägte absolute Barriere existiert und ein Vorkomplex die Temperaturabhängigkeit beeinflusst. Die Werte der Geschwindigkeitskonstante für verschiedene Temperaturen sind mit wichtigen experimentellen Bedingungen in Tabelle zusammengefasst 4.3 . 


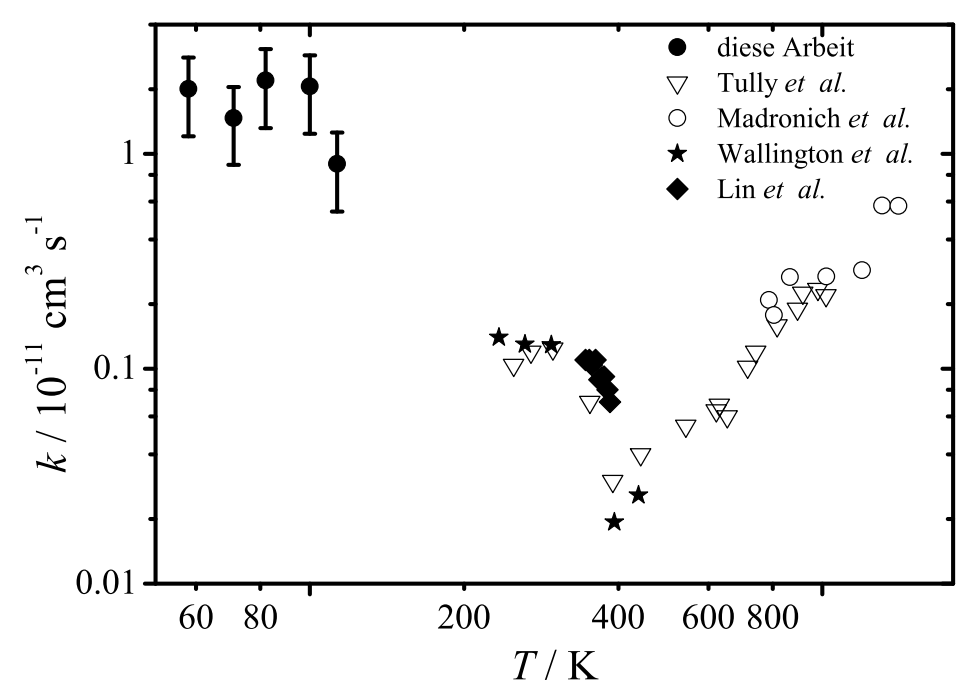

Abbildung 4.5: Die Geschwindigkeitskonstante der Reaktion zwischen Benzol und $\mathrm{OH}$ ist zusammen mit Literaturdaten aus den Arbeiten von Tully et al. [120], Madronich et al. [121], Wallington et al. [122] und Lin et al. [123] dargestellt.

$\begin{array}{lcccc}\text { Düse } & T / \mathrm{K} & \rho_{\text {ges }} / 10^{17} \mathrm{~cm}^{-3} & k / 10^{-11} \mathrm{~cm}^{3} \mathrm{~s}^{-1} & {[\text { Benzol }]_{\max } / 10^{14} \mathrm{~cm}^{-3}} \\ \text { M211E17 } & 113 & 1.0 & (0.90 \pm 0.36) & 5 \\ \text { M278E16 } & 100 & 0.5 & (2.06 \pm 0.82) & 1.4 \\ \text { M351E17 } & 82 & 0.9 & (2.20 \pm 0.88) & 2.5 \\ \text { M408E16 } & 71 & 0.9 & (1.47 \pm 0.58) & 2 \\ \text { M508E16 } & 58 & 0.5 & (2.01 \pm 0.80) & 1.7\end{array}$

Tabelle 4.3: Geschwindigkeitskonstanten für die Reaktion des OH-Radikals mit Benzol: Die angegebenen Geschwindigkeitskonstanten sind der Mittelwert aus mindestens drei Einzelmessungen. 


\subsection{Die Reaktion von Alaninethylester mit $\mathrm{OH}$}

Neben der Atmosphärenchemie spielt das OH-Radikal auch in wässriger Lösung eine Rolle bei der Entstehung von oxidativem Stress von lebenden Zellen. $\mathrm{OH}$ ist zu einer Klasse von Teilchen zu zählen, die unter dem Oberbegriff „reactive oxigen species" kurz ROS zusammengefasst werden. Diese Gruppe von Radikalen und Oxidationsmitteln wird für Schädigungen von Zellgewebe verantwortlich gemacht, die mit dem Alterungsprozess in Zusammenhang gebracht werden.

Der Angriff eines Radikals auf ein Protein ist näherungsweise durch den Angriff auf eine seiner Aminosäuren zu beschreiben. Wegen des sehr geringen Dampfdrucks der freien Aminosäure wurde in dieser Arbeit der Ethylester des Alanins als Modellverbindung verwendet.

Zur Reaktion des OH-Radikals mit Aminosäuren gibt es eine Reihe von Untersuchungen in wässriger Phase. Noch besteht über den genauen Mechanismus dieser Reaktion keine Einigkeit: Verschiedene Untersuchungen kommen zu unterschiedlichen Ergebnissen bezüglich der Reaktionsprodukte. Eine Studie, die den OH-vermittelten ${ }^{1} \mathrm{H} /{ }^{2} \mathrm{H}$-Austausch von Wasserstoffatomen in Aminosäuren mit Magnetresonanzspekroskopie verfolgte, kommt zu dem Schluss, dass die Seitenketten der Aminosäuren der bevorzugte Ort der Reaktion sind [124]. Andere Untersuchungen haben C $\alpha$ oder Stickstoffzentrierte Radikale als Produkte nachgewiesen [125].

Zur Reaktion von Aminosäuren mit $\mathrm{OH}$ in der Gasphase gibt es lediglich theoretische Arbeiten [16]. Die Reaktion von $\mathrm{OH}$ mit Alanin und Glycin wird darin mit Dichtefunktionaltheorie und MP2 Methoden untersucht, die Energie einzelner Strukturen wird mit höheren Methoden berechnet. Dabei kommen Galano et al. zu dem Ergebnis, dass die Reaktion zu einer Abstraktion eines Wasserstoffatoms an $\mathrm{C} \alpha$ - oder $\mathrm{C} \beta$-Position führt. Laut diesen Berechnungen ist die Aktivierungsenergie für das Wasserstoffatom an $\mathrm{C} \alpha$-Position etwas geringer. Für beide Reaktionskanäle bildet sich vor der eigentlichen Abstraktion je ein Komplex zwischen dem Radikal und Alanin aus, der für beide Konfigurationen eine Stabilisierungsenergie von etwa $8 \mathrm{kcal} / \mathrm{mol}$ aufweist. Schematisch ist die Reaktionskoordinate der Abstraktion in Abbildung 4.6 dargestellt. Die Carboxylgruppe spielt bei der Reaktion keine 
Rolle, es wurden trotz der Suche nach Komplexen zwischen dem Radikal und der Carboxylgruppe keine solchen Strukturen gefunden.

Obwohl die Rechnungen sich auf die freie Aminosäure beziehen, sind für den hier experimentell untersuchten Ethylester des Alanins Parallelen zu erwarten, da die Carboxylgruppe nach den Berechnungen von Galano et al. keine direkte Bedeutung für das Reaktionsgeschehen hat. Der Ethylester ist in dieser Arbeit verwendet worden, weil die freie Aminosäure einen sehr viel geringeren Dampfdruck aufweist und mit der experimentellen Methode dieser Arbeit bislang nicht untersucht werden kann. Allerdings bestätigen vorläufige Ergebnisse der Rechnungen mit dem Ethylester, dass die Estergruppe den Reaktionsmechanismus nicht maßgeblich beeinflusst [126].

Abbildung 4.7 zeigt einen typischen Verlauf der Fluoreszenzintensität für die Reaktion von $\mathrm{OH}$ mit Alaninethylester unter Bedingungen pseudo-erster Ordnung. Die so gewonnenen Geschwindigkeitskonstanten werden gegen die Konzentration des Alaninethylesters aufgetragen, wie in Abbildung 4.8 dargestellt. Die temperaturabhängige Geschwindigkeitskonstante der Reaktion des Alaninethylesters mit $\mathrm{OH}$ ist schließlich in Abbildung 4.9 dargestellt, in Tabelle 4.4 sind sie mit wichtigen experimentellen Bedingungen aufgelistet. Die Geschwindigkeitskonstante zeigt eine ausgeprägte negative Temperaturabhängigkeit, was mit dem von Galano et al. gefundenen Energieprofil für die H-Abstraktion in $\alpha$-oder $\beta$-Position vereinbar ist (vgl. Abbildung 4.6). Die Reaktion ist wahrscheinlich nicht einfanglimitiert, da in diesem Fall eine schwächere Temperaturabhängigkeit zu beobachten wäre. Die relativ niedrige Geschwindigkeitskonstante bei Raumtemperatur spricht ebenfalls dagegen. Eine Barriere deutlich oberhalb der Energie der Edukte kann wegen der negativen Temperaturabhängigkeit der Geschwindigkeitskonstante ausgeschlossen werden. Die experimentellen Daten legen nahe, dass die Barriere zwischen den Wasserstoffbrücken-gebundenen Komplexen und den Produkten unterhalb der Energie der Edukte liegt. Die Temperaturabhängigkeit wird dann durch die konkurrierenden Zerfallskanäle des Wasserstoffbrücken-gebundenen Komplexes bestimmt, der Edukte und den inneren Übergangszustand trennt. Schematisch ist die Behandlung einer solchen Konkurrenz im Abschnitt 2.2.5 für allgemeine Fälle diskutiert worden. Bei der Konkurrenz entscheidet die Energie- und Drehimpulsabhängigkeit der Zerfalls- 


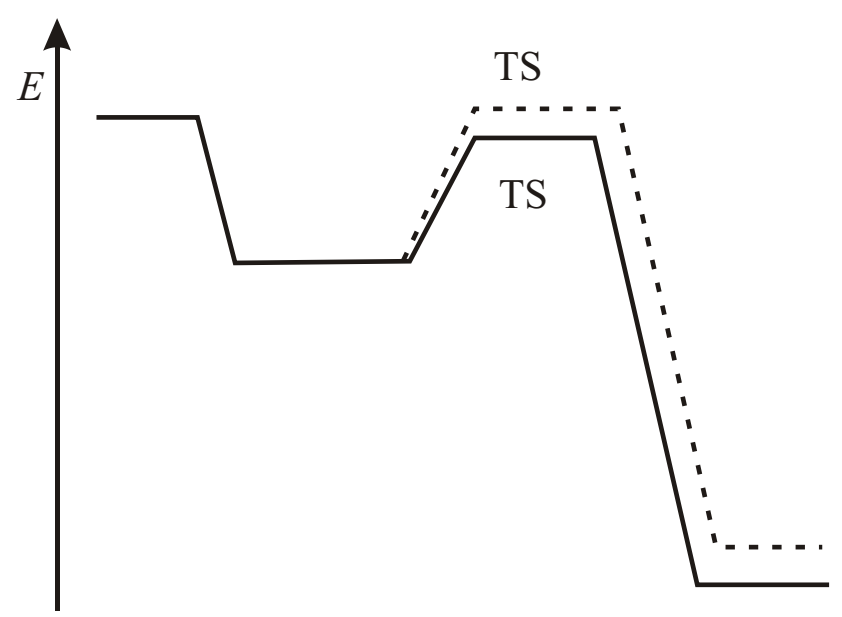

Abbildung 4.6: Die schematisierte Reaktionskoordinate, wie in Referenz [16] berechnet. Die Energien sind nicht maßstabsgerecht abgebildet. TS $\alpha$ bezeichnet den Übergangszustand für die $\mathrm{H}$-Abstraktion an $\mathrm{C} \alpha$ Position, TS $\beta$ die entsprechende Abstraktion an $\mathrm{C} \beta$-Position.

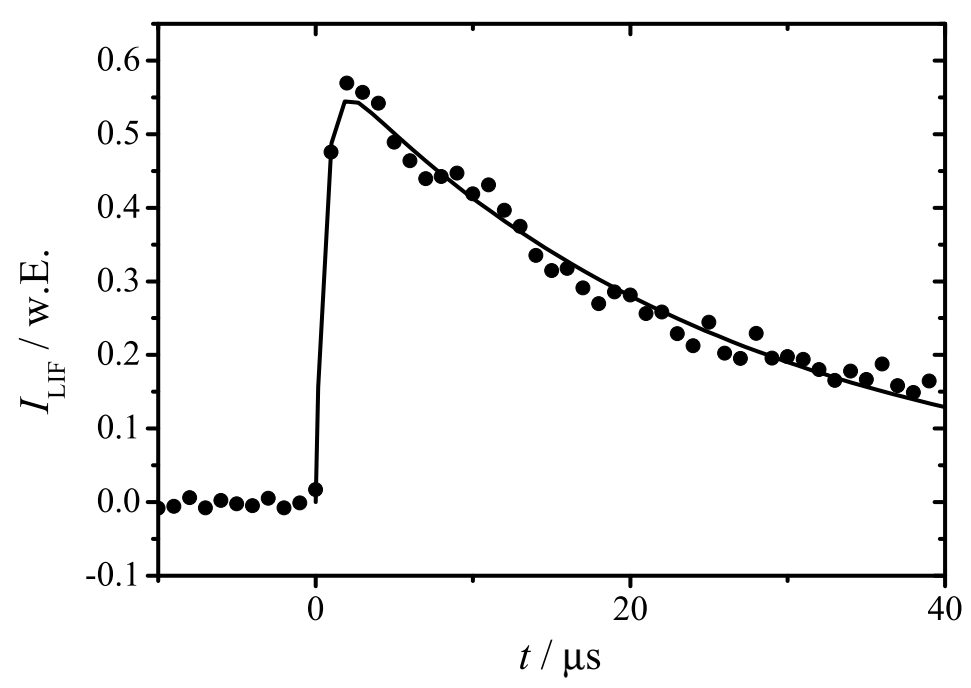

Abbildung 4.7: Das Fluoreszenzsignal von $\mathrm{OH}$ zeigt eine Zeitabhängigkeit pseudoerster Ordnung, hier dargestellt für $\rho_{\text {ges }}=0.9 \cdot 10^{17} \mathrm{~cm}^{-3}, T=91 \mathrm{~K}$ und $\rho_{\text {Alaninethylester }}=3.9 \cdot 10^{12} \mathrm{~cm}^{-3}$ 


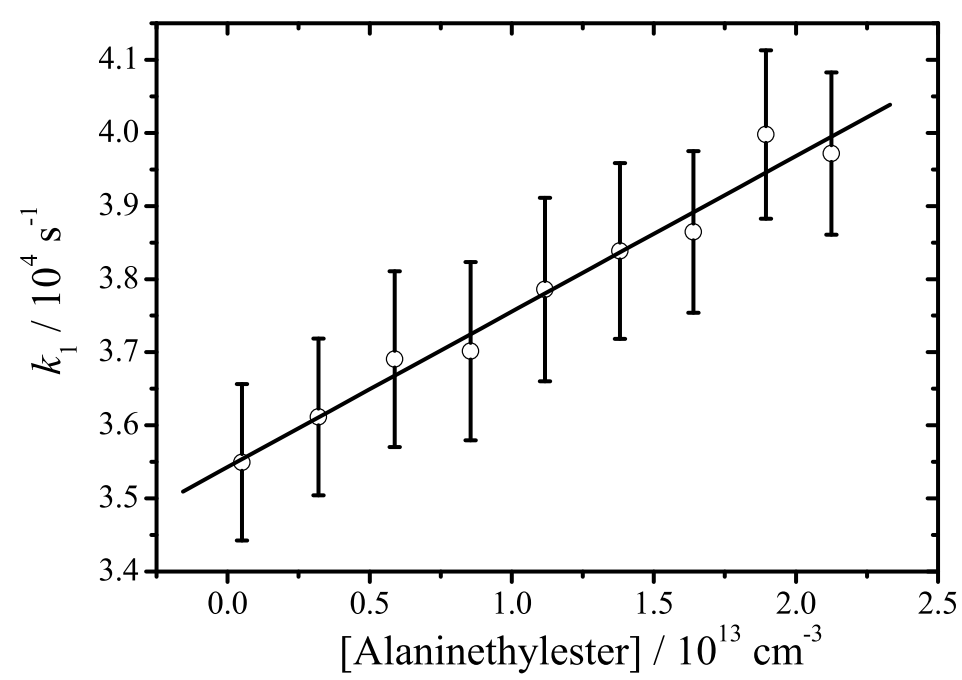

Abbildung 4.8: Die Geschwindigkeitskonstanten pseudo-erster Ordnung werden gegen die Alaninethylesterkonzentration aufgetragen. Diese Einzelmessung ergibt eine Geschwindigkeitskonstante von $2.1 \pm 0.5$ • $10^{-10} \mathrm{~cm}^{3} \mathrm{~s}^{-1}$ für $91 \mathrm{~K}$ und $\rho_{\text {ges }}=0.9 \cdot 10^{17} \mathrm{~cm}^{-3}$

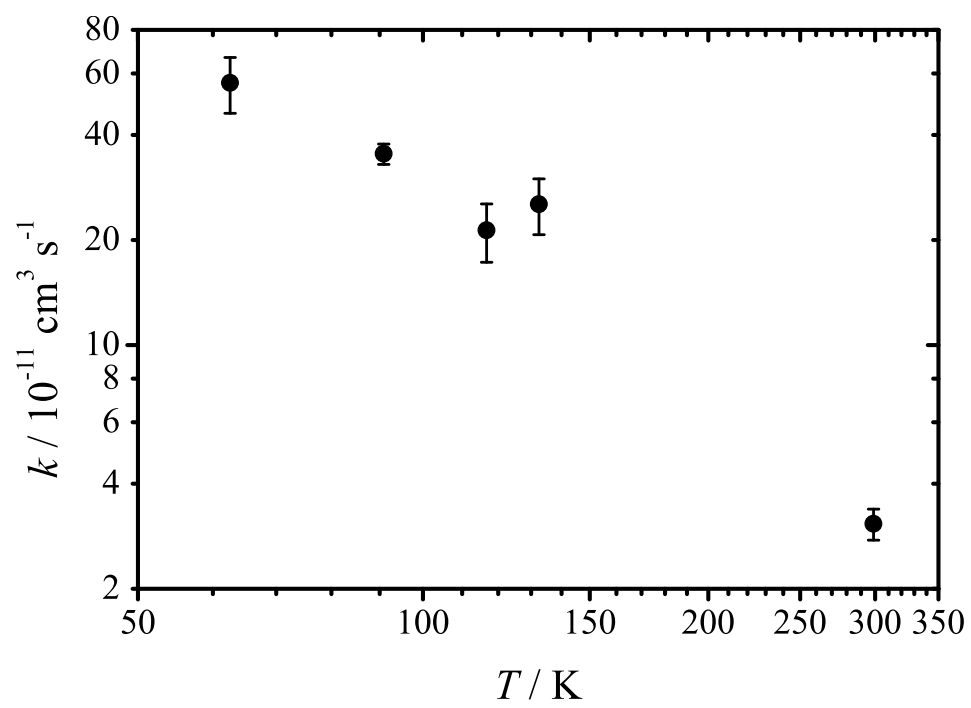

Abbildung 4.9: Auftragung der Geschwindigkeitskonstante der Reaktion von $\mathrm{OH}$ mit Alaninethylester 
konstanten zurück zu den Edukten und vorwärts zu den Produkten der Abstraktion über die Temperaturabhängigkeit der Reaktion. Die obere Grenze der Geschwindigkeitskonstante ist durch eine Einfanggeschwindigkeitskonstante gegeben. Bei der Berechnung der Einfanggeschwindigkeit sollte die Anisotropie der Wechselwirkung berücksichtigt werden.

Da die Energien des Komplexes und der inneren Übergangszustände für die HAbstraktion in $\alpha$ - oder $\beta$-Position sehr ähnlich sind, wirken sich die zwei verschiedenen Positionen der Abstraktion in der Temperaturabhängigkeit der hier vorgestellten Daten nicht sichtbar aus. Wenn die Energie der beiden Übergangszustände auch beim Ester nur eine geringe Differenz aufweisen, sollten beide primären Produkte gebildet werden. Das Produkt der Abstraktion an $\mathrm{C} \alpha$ ist deutlich stabiler als das Radikal, was aus der Abstraktion an $\mathrm{C} \beta$ resultiert. Eine Isomerisierung des weniger stabilen $\mathrm{C} \beta$-Produkts wäre im Anschluss an die Abstraktionsreaktion denkbar.

Abschließend sollen noch mögliche systematische Fehler bei der Ermittlung der Geschwindigkeitskonstante diskutiert werden. Die Auftragung der Geschwindigkeitskonstante in Abbildung 4.8 weist im verwendeten Konzentrationsbereich keine Anzeichen auf eine Dimerbildung der Alaninestermoleküle in kalten Düsenstrahl auf. Die Bildung von Dimeren und kleinen Clustern des Esters würde sich als eine Abweichung von der linearen Abhängigkeit zwischen der Geschwindigkeitskonstante pseudo-erster Ordnung und der Esterkonzentration bemerkbar machen. Eine genauere Betrachtung der Bildung von kleinen Clustern ist mit FTIR-Technik in einer freien Expansion von Hesse untersucht worden [127]. Natürlich wurde mit der Wahl des Konzentrationsbereichs des Esters der möglichen Kondensation Rechnung getragen, so dass die Kondensation der Reaktionspartner eine untergeordnete Rolle spielt.

Der Ester neigt als Reinstoff zur Oligomerisierung. Daher wurde er bis zur Verwendung bei $-20^{\circ} \mathrm{C}$ gelagert. Die Produkte der Oligomerisierung, Ethanol und Alaninoligomere sind vergleichsweise unreaktiv oder nicht flüchtig. Damit würde die Geschwindigkeitskonstante mit fortschreitendem Zerfall des Esters scheinbar kleiner werden. Bei der Durchführung der Experimente wurden die Gasmischungen mit dem Ester deshalb nach einer maximalen Zeit von zehn Tagen verworfen. Es konnte keine Korrelation zwischen dem Alter der vorbereiteten Gasmischung und der 


$\begin{array}{lcccc}\text { Düse } & T / \mathrm{K} & \rho_{\text {ges }} / 10^{17} \mathrm{~cm}^{-3} & k / 10^{-10} \mathrm{~cm}^{3} \mathrm{~s}^{-1} & \text { Anzahl Messungen } \\ - & 298 & 3.4 & (0.310 \pm 0.031) & 5 \\ \text { M211E17 } & 133 & 1.3 & (2.53 \pm 0.46) & 5 \\ \text { M278E16 } & 117 & 0.7 & (2.13 \pm 0.40) & 7 \\ \text { M351E17 } & 91 & 1.1 & (3.53 \pm 0.24) & 5 \\ \text { M508E16 } & 60 & 0.6 & (5.64 \pm 1.0) & 3\end{array}$

Tabelle 4.4: Geschwindigkeitskonstanten und experimentelle Bedingungen für die Reaktion des OH-Radikals mit Alaninethylester: Die maximale Konzentration des Esters in der Expansion betrug bei Raumtemperatur $3 \cdot 10^{13} \mathrm{~cm}^{-3}$ und bei den tiefen Temperaturen zwischen 2 . $10^{13} \mathrm{~cm}^{-3}(134 \mathrm{~K})$ und $1 \cdot 10^{13} \mathrm{~cm}^{-3}(62 \mathrm{~K})$

Geschwindigkeitskonstante festgestellt werden. Zudem konnten im NMR-Spektrum des Esters keine Ethanolspuren gefunden werden [107].

\subsection{Die Reaktion von Acetaldehyd mit $\mathrm{OH}$}

Die Ergebnisse zur Untersuchung der Reaktion von Acetaldehyd mit $\mathrm{OH}$ in der Göttinger CRESU-Apparatur sind kürzlich veröffentlicht worden [27]. Neben dem Artikel in gedruckter Form steht ergänzendes Online-Material zur Verfügung, das die Berechnung der Geschwindigkeitskonstanten, die ab initio Strukturen, die kinetischen Daten und den experimentellen Aufbau genauer beschreibt [128]. Ein Auszug der hier vorgestellten Daten ist zudem schon in der Diplomarbeit von Esteban Vöhringer-Martinez vorgestellt worden [129], allerdings stehen jetzt weitere theoretische und experimentelle Ergebnisse zur Verfügung.

Die Reaktion ist temperaturabhängig in Gegenwart von etwa 3\% Stoffmengenanteil Wasserdampf und ohne Wasserdampf untersucht worden. Zunächst wird die wasserfreie Reaktion mit ihrer Temperaturabhängigkeit, den quantenmechanischen Rechnungen und einem Vergleich mit bereits veröffentlichten Daten diskutiert. In einem zweiten Schritt wird der Einfluss von einzelnen Wassermolekülen auf die Reaktion besprochen. 


\section{Die Reaktion von Acetaldehyd und $\mathrm{OH}$ ohne Wasser}

Die Reaktion von Acetaldehyd und dem OH-Radikal läuft beinahe ausschließlich über die Abstraktion des aldehydischen Wasserstoffs ab. Unabhängige Produktstudien kommen $\mathrm{zu}$ dem Ergebnis, dass $\mathrm{CH}_{3} \mathrm{CO}$ mit einer Primärausbeute von mindestens $95 \%$ bei Raumtemperatur [5, 6] und darunter [7] gebildet wird. Kürzlich ist eine Arbeit von Taylor et al. erschienen, nach der für Temperaturen bis $600 \mathrm{~K}$ das aldehydische Wasserstoffatom bevorzugt abstrahiert wird, aber bei $860 \mathrm{~K}$ bereits die Abstraktion eines Wasserstoffatoms der Methylgruppe dominiert [130]. Diese Ergebnisse stützen sich auf Untersuchungen des kinetischen Isotopeneffekts bei diesen Temperaturen. Neben den experimentellen Studien sind von Alvarez-Idaboy et al. [131] und von D'Anna et al. [6] theoretische Untersuchungen zu wichtigen Strukturen in der Reaktionskoordinate der Reaktion vorgenommen worden.

Die Energien der Übergangszustände einer $\mathrm{OH}$-Addition und einer $\mathrm{H}_{\text {Methyl- }} \mathrm{Ab}$ straktion liegen im Gegensatz zur Abstraktion von $\mathrm{H}_{\text {Aldehyd }}$ oberhalb der Energie der isolierten Edukte. Damit bestätigen die Berechnungen die experimentellen Produktstudien bezüglich des Ortes der H-Abstraktion. Die Abwesenheit einer absoluten Barriere in den Rechnungen weist bereits auf die negative Temperaturabhängigkeit hin, genauso wie die bereits gefundenen Wasserstoffbrücken gebundenen Komplexe zwischen Acetaldehyd und OH. Selbst der Verlauf der Geschwindigkeitskonstante bei hohen Temperaturen und die Ergebnisse zum kinetischen Isotopeneffekt von Taylor et al. werden damit untermauert. Die Berechnungen der Geschwindigkeitskonstante der Reaktion gehen allerdings bei beiden theoretischen Arbeiten von der Beschreibung durch einen einzigen Übergangszustand aus und können die Temperaturabhängigkeit nur durch die Einführung eines Tunnelpotentials an die experimentellen Ergebnisse anpassen.

Die Geschwindigkeitskonstante für die Abstraktionsreaktion zwischen $\mathrm{OH}$ und Acetaldehyd ist zusammen mit ausgewählten experimentellen Daten anderer Arbeitsgruppen in Abbildung 4.12 dargestellt. In den Abbildungen 4.10 und 4.11 finden sich die zugehörigen Auftragungen zur Ermittlung der Geschwindigkeitskonstanten pseudo-erster und zweiter Ordnung. Aus der Auftragung der Geschwindigkeitskonstanten pseudo-erster Ordnung ist eine leichte Abweichung vom linearen Verhalten 
erkennbar, die auf die einsetzende Kondensation des Acetaldehyds zu kleinen Clustern zurückzuführen ist. Die Grenzgerade niedriger Acetaldehydkonzentration entspricht daher der tatsächlichen Geschwindigkeitskonstante. Die Korrektur erfolgt über die Berücksichtigung der Kondensation als bimolekularem Prozess, der im Laufe der Reaktionszeit die effektive Konzentration von Acetaldehyd vermindert. Diese Korrektur ist für die Geschwindigkeitskonstanten unter $100 \mathrm{~K}$ durchgeführt worden. Die Geschwindigkeitskonstante weist bei etwa $550 \mathrm{~K}$ ein Minimum auf, was

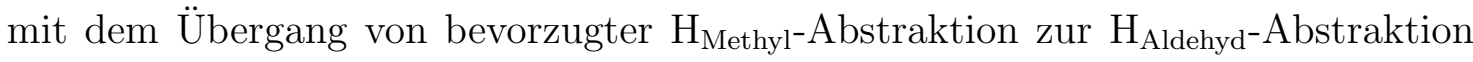
mit sinkender Temperatur zu erklären ist. Die negative Temperaturabhängigkeit die bereits von Michael et al. [132] und Sivakumaran et al. [133] für höhere Temperaturen beschrieben wird, setzt sich zu tieferen Temperaturen fort, allerdings weichen die Absolutwerte der Geschwindigkeitskonstanten leicht von der Extrapolation der Daten höherer Temperatur ab. Bei Raumtemperatur ist die Übereinstimmung ausgezeichnet.

In keiner bisher veröffentlichten Arbeit wurde für die Reaktion eine Druckabhängigkeit im Bereich zwischen 1 und etwa 1000 mbar gefunden. Die Lebensdauer von intermediären Komplexen in der Reaktion wird dadurch eingegrenzt, da ihre Stabilisierung in der Druckabhängigkeit der Reaktion sichtbar werden würde.

Basierend auf den ab initio Strukturen, die in Kooperation mit J. Franciscos Arbeitsgruppe berechnet wurden, ergibt sich eine Erklärungsmöglichkeit für die negative Temperaturabhängigkeit der Reaktion, die experimentelle und theoretische Kenntnisse berücksichtigt und mit ihnen im Einklang steht. Bereits in der Arbeit von Alvarez-Idaboy ist die Bedeutung von schwach gebundenen Vorkomplexen bei der Reaktion betont worden, auch wenn die Autoren dieser Arbeit sie bei der Berechnung der Geschwindigkeitskonstanten nicht adäquat berücksichtigen.

Abbildung 4.13 zeigt das Energieprofil der Reaktion. Die Konfiguration der beteiligten Strukturen kann der Abbildung 4.14 entnommen werden. Diese Strukturen sind auf dem MP2-Niveau mit dem aug-cc-pVDZ Basissatz mit dem Gaussian98 Softwarepaket [134] berechnet worden. Die Energien dieser charakteristischen Punkte der Potentialfläche wurden mit CCSD(T) mit dem cc-pVTZ-Basissatz berechnet.

Die isolierten Edukte bilden zunächst einen Komplex, der eine Stabilisierungsenergie von $19.9 \mathrm{~kJ} / \mathrm{mol}$ ausweist (Struktur A in Abbildung 4.14), der Übergangszu- 


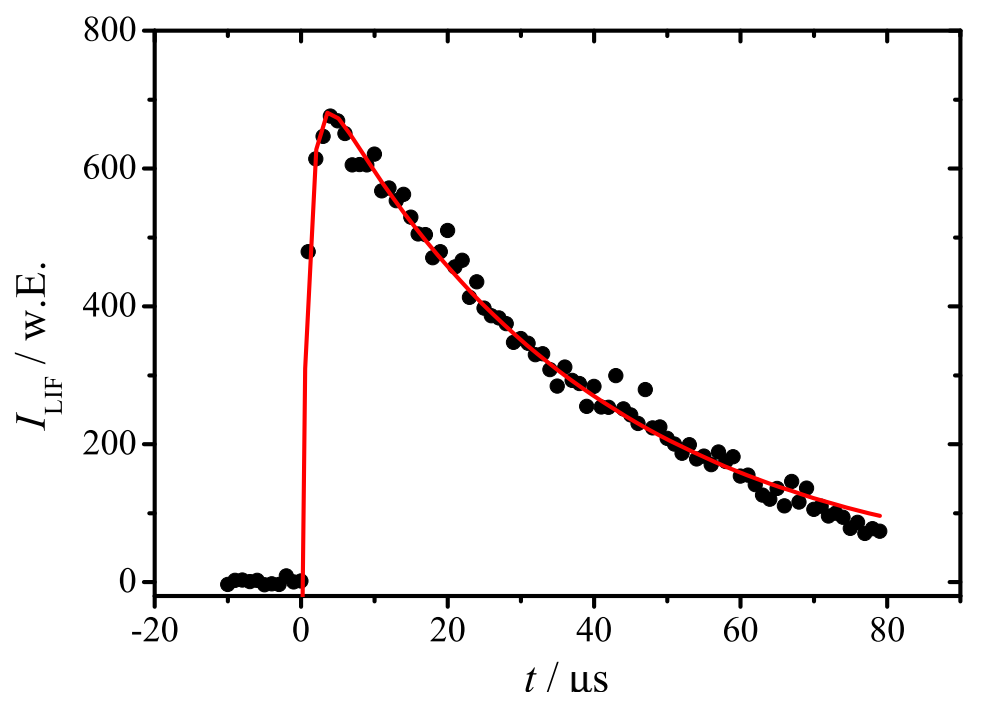

Abbildung 4.10: Das Fluoreszenzsignal von $\mathrm{OH}$ zeigt eine Zeitabhängigkeit pseudoerster Ordnung, hier dargestellt für $\rho_{\text {ges }}=0.85 \cdot 10^{17} \mathrm{~cm}^{-3}, T=$ $77 \mathrm{~K}$ und $\rho_{\text {Acetaldehyd }}=1.6 \cdot 10^{14} \mathrm{~cm}^{-3}$.

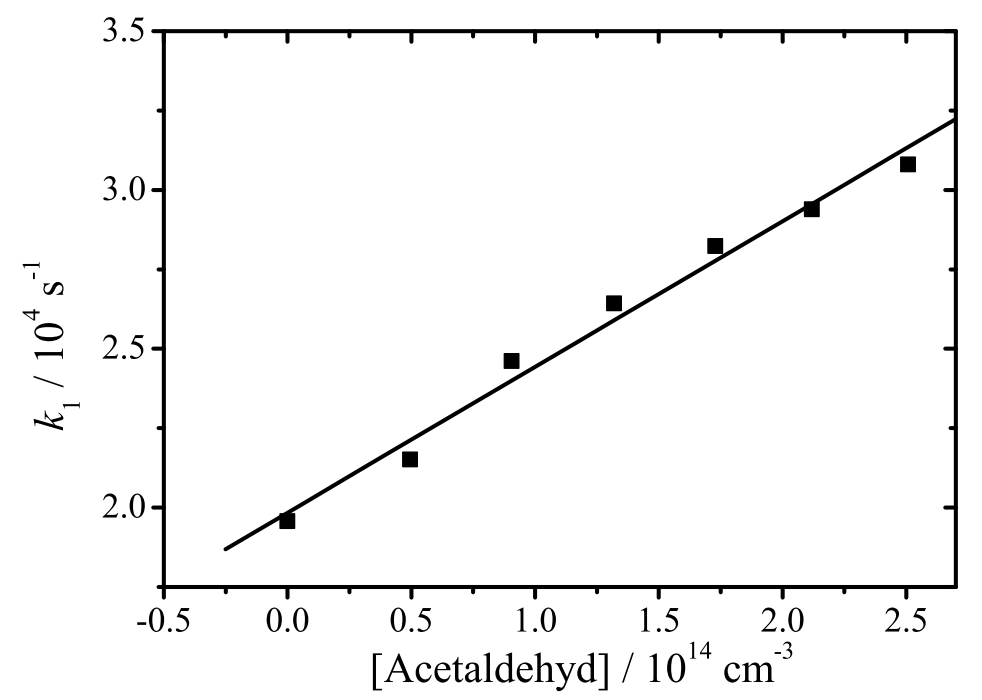

Abbildung 4.11: Die Geschwindigkeitskonstanten pseudo-erster Ordnung werden gegen die Acetaldehydkonzentration aufgetragen. Diese Einzelmessung ergibt eine Geschwindigkeitskonstante von $5.5 \pm 0.5$. $10^{-11} \mathrm{~cm}^{3} \mathrm{~s}^{-1}$ für $77 \mathrm{~K}$ und $\rho_{\text {ges }}=0.85 \cdot 10^{17} \mathrm{~cm}^{-3}$. 


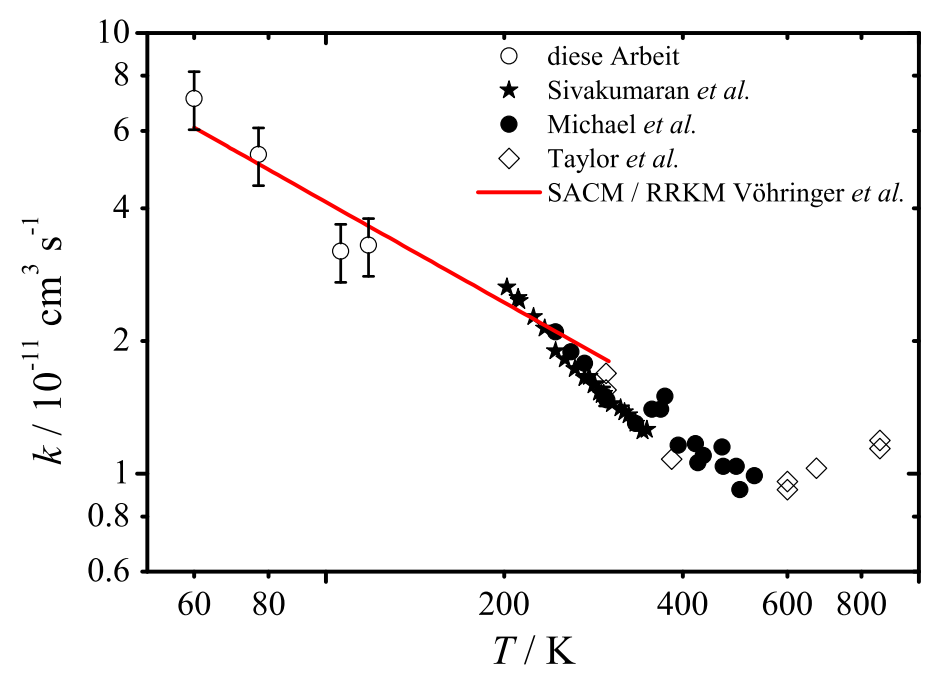

Abbildung 4.12: Die Temperaturabhängigkeit der Reaktion zwischen $\mathrm{OH}$ und Acetaldehyd zusammen mit Daten von Sivakumaran et al. [133], Michael et al. [132], Taylor et al. [130] und Vöhringer et al. [27]

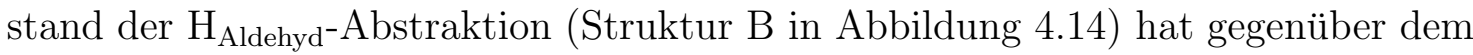
Komplex eine Barriere von $8 \mathrm{~kJ} / \mathrm{mol}$ in Übereinstimmung mit D'Anna und AlvarezIdaboy. Neben dem Komplex A, der direkt zum Übergangszustand am Barrierenmaximum B führt, gibt es ein weiteres Isomer eines Acetaldehyd-OH Komplexes, das eine Konfiguration aufweist, die zur Abstraktion eines Methyl-Wasserstoffs führt. Diese beiden Strukturen können durch die Rotation des OH-Radikals ineinander isomerisieren.

Die Situation aus der Perspektive des Vorkomplexes A entspricht formal der eines lockeren und eines festen Übergangszustands der beiden Zerfallskanäle. Wie im Abschnitt 2.2.5 für einen allgemeinen Fall beschrieben, entscheidet in einer solchen Situation die Energie und Drehimpulsabhängigkeit der spezifischen Geschwindigkeitskonstanten beider Kanäle über die Temperaturabhängigkeit der Reaktion, und kann die ausgeprägte negative Temperaturabhängigkeit erklären. Die in Abbildung 4.12 abgebildeten SACM/RRKM-Rechnungen, deren Parameter in Referenz [128] veröffentlicht sind, greifen exakt auf die Methodik der oben erwähnten Konkurrenz zurück. 


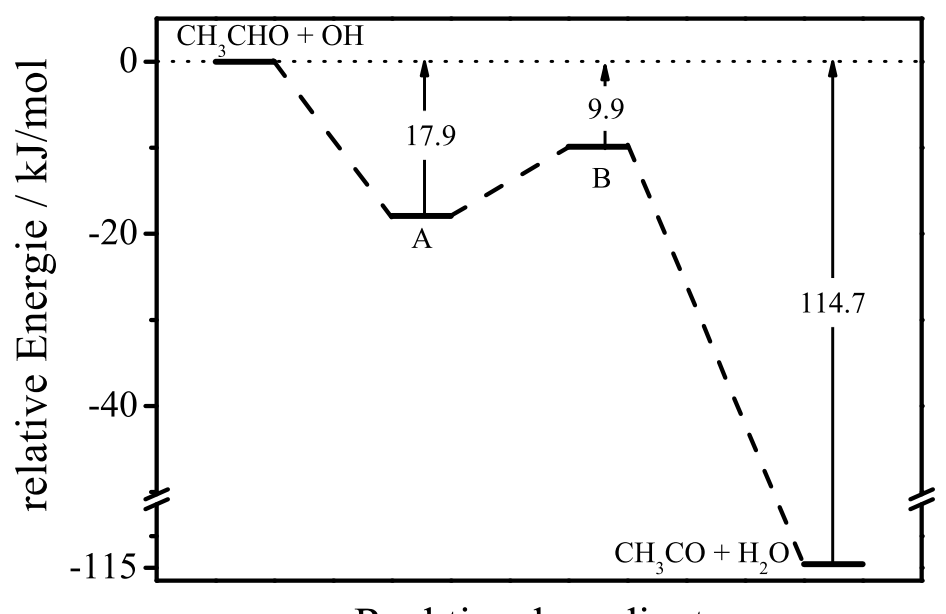

Reaktionskoordinate

Abbildung 4.13: Das Energieprofil der Reaktion von Acetaldehyd und $\mathrm{OH}$ weist auf die Bedeutung des Vorkomplexes A hin, der auf der Reaktionskoordinate zwischen den Edukten und dem Übergangszustand der $\mathrm{H}_{\text {Aldehyd }}$-Abstraktion B liegt. Die Energien sind die Ergebnisse von single-point Berechnungen mit $\operatorname{CCSD}(\mathrm{T})$ mit dem cc-pVTZBasissatz.

Dabei ist die Geschwindigkeitskonstante für den lockeren Übergangszustand mit einem SACM-Ansatz beschrieben worden und der Zerfall über den festen Übergangszustand mit einer Geschwindigkeitskonstante nach RRKM. Für die Nettogeschwindigkeitskonstante $k$ ergibt sich, wie im Abschnitt 2.2.5 erläutert

$$
k=\frac{k_{\mathrm{B}}}{h}\left(\frac{Q_{\mathrm{A}}}{Q_{\text {Acetaldehyd }} Q_{\mathrm{OH}}}\right)_{\text {el, trans }}\left(\frac{Q_{\mathrm{A}}^{*} Y}{Q_{\text {Acetaldehyd }} Q_{\mathrm{OH}}}\right)_{\text {vib, rot }}
$$

mit

$$
Q_{\mathrm{A}}^{*} Y=\sum_{J=0}^{\infty}(2 J+1) \int_{0}^{\infty} \frac{\mathrm{d} E}{k_{\mathrm{B}} T} \exp \left(-\frac{E}{k_{\mathrm{B}} T}\right) W_{\mathrm{a}}(E, J) \cdot\left[\frac{W_{\mathrm{b}}(E, J)}{W_{\mathrm{a}}(E, J)+W_{\mathrm{b}}(E, J)}\right]
$$




$\begin{array}{lcccc}\text { Düse } & T / \mathrm{K} & \rho_{\text {ges }} / 10^{17} \mathrm{~cm}^{-3} & k / 10^{-11} \mathrm{~cm}^{3} \mathrm{~s}^{-1} & \text { Anzahl Messungen } \\ - & 295 & 2.6 & (1.5 \pm 0.08) & 3 \\ \text { M211E17 } & 118 & 1.1 & (3.3 \pm 0.50) & 6 \\ \text { M278E16 } & 106 & 0.75 & (3.2 \pm 0.48) & 7 \\ \text { M351E17 } & 77 & 0.85 & (5.3 \pm 0.79) & 6 \\ \text { M508E16 } & 60 & 0.65 & (7.1 \pm 1.0) & 6\end{array}$

Tabelle 4.5: Geschwindigkeitskonstanten und experimentelle Bedingungen für die Reaktion des OH-Radikals mit Acetaldehyd: Die maximale Konzentration von Acetaldehyd in der Expansion betrug bei Raumtemperatur $15 \cdot 10^{14} \mathrm{~cm}^{-3}$ und bei den tiefen Temperaturen zwischen 7 . $10^{14} \mathrm{~cm}^{-3}(118 \mathrm{~K}, 106 \mathrm{~K})$ und $2.5 \cdot 10^{14} \mathrm{~cm}^{-3}(77 \mathrm{~K}, 60 \mathrm{~K})$.

und

$$
Y=\left[\frac{W_{\mathrm{b}}(E, J)}{W_{\mathrm{a}}(E, J)+W_{\mathrm{b}}(E, J)}\right] .
$$

Die Zustandssummen des Vorkomplexes A sind mit $Q_{\mathrm{A}}$ bezeichnet, die Zustandssummen der Edukte mit $Q_{\text {Acetaldehyd }}$ und $Q_{\mathrm{OH}}$. Der Verzweigungsfaktor $Y$ ergibt sich als Quotient der Anzahl offener Kanäle für den Übergangszustand zwischen Edukten und Vorkomplex $W_{\mathrm{a}}$ und der Anzahl offener Kanäle am Übergangszustand B $W_{\mathrm{b}}$.

Die Lebensdauer des Vorkomplexes A kann anhand der beiden Zerfallskonstanten berechnet werden. Die erhaltene Lebensdauer liegt im unteren Pikosekundenbereich, was auch die experimentell nicht beobachtete Druckabhängigkeit erklärt.

Die Reaktion zwischen Acetaldehyd und $\mathrm{OH}$ ist somit von theoretischer und experimenteller Seite genau betrachtet worden, wobei sich ein Gesamtbild ohne widersprüchliche Aussagen ergibt, das die bestehenden Erkenntnisse zu dieser Reaktion plausibel erklärt. Allerdings zeigen zusätzliche Messungen zur Schwingungsdesaktivierung von $\mathrm{OH}(\mathrm{v}=1)$ durch Acetaldehyd eine ungewöhnlich starke negative Temperaturabhängigkeit, die genauer untersucht werden sollte. 
A
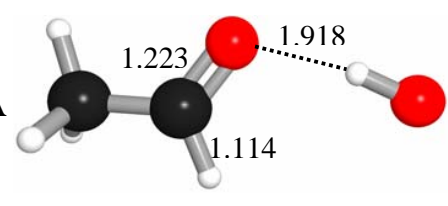

C

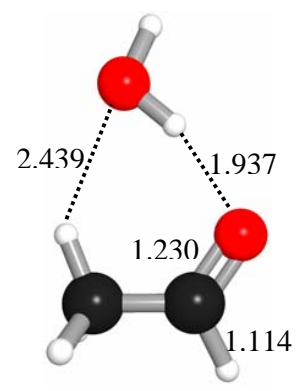

$\mathrm{E}$

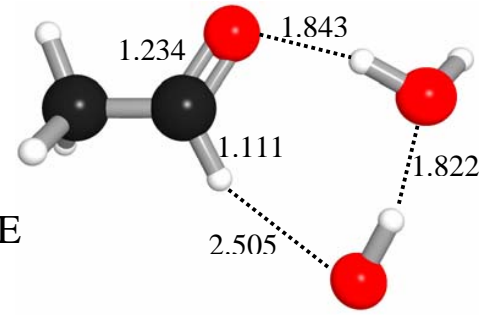

B

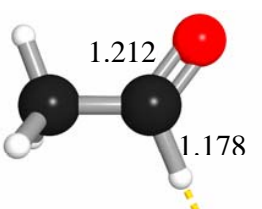

1.460

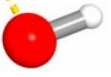

D

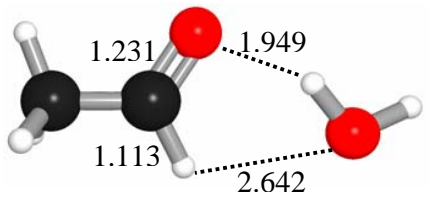

$\mathrm{F}$

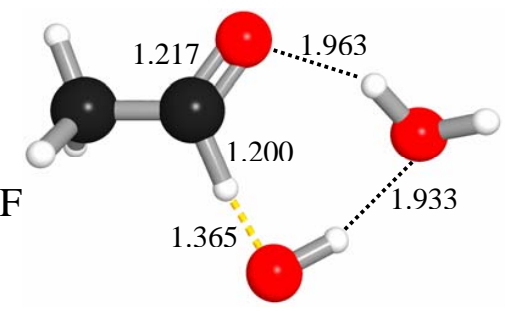

Abbildung 4.14: ab initio Strukturen von Acetaldehyd, OH und Wasser: Die Strukturen sind auf der Basis von MP2-Berechnungen mit dem aug-ccpVDZ Basissatz ermittelt worden. Die Übergangszustände wurden durch Frequenzberechnungen auf diesem Niveau als Sattelpunkte erster Ordnung bestätigt. Struktur A ist der Vorkomplex des wasserfreien Reaktionspfades, B der entsprechende Übergangszustand. Die Wasserkomplexe C und D treten bei der Zugabe von Wasser zum Reaktionsgemisch auf. E ist der Vorkomplex des Reaktionpfades mit Wasser, F der entsprechende Übergangszustand. 


\section{Die Reaktion von Acetaldehyd mit $\mathrm{OH}$ in Gegenwart von Wasserdampf}

Wie weiter oben erwähnt ist die Reaktion auch in Gegenwart von $3 \%$ Wasserdampf im Reaktionsgemisch untersucht worden. Seit einiger Zeit wird darüber spekuliert, welchen Einfluss einzelne Wassermoleküle auf die Reaktionen insbesondere von Radikalen in der Gasphase haben können. Die unterschiedlichen Möglichkeiten eines Einflusses von Wassermolekülen auf chemische Reaktionen sind in der Einleitung bereits angesprochen worden. Die Reaktion von Acetaldehyd und Wasser bietet wegen des relativ genau bekannten Mechanismus die Möglichkeit, den Einfluss von Wassermolekülen auf die Geschwindigkeitskonstante und das zugrundeliegende Potential der Reaktion zu untersuchen. Die Energietransfereigenschaften von Wasser spielen bei dieser Reaktion, die ohne langlebige Intermediate und unter Beteiligung Wasserstoffbrücken gebundener Komplexe abläuft, keine wichtige Rolle für die Reaktionsgeschwindigkeit. Wie in der Einleitung bereits erwähnt, trägt der geringe Wasseranteil (3\%) zum Energietransfer in gleichem Maße wie das Trägergas Stickstoff $(96 \%)$ bei. Die Reaktion zwischen Acetaldehyd und $\mathrm{OH}$ zeigt bis zu einem Druck von 1000 mbar keine Druckabhängigkeit. Daher ist durch den verwendeten Wasseranteil in den Experimenten keine Veränderung der Geschwindigkeitskonstante über den Energietransfer in Stößen zu erwarten.

Die Messungen in Gegenwart von Wasserdampf zeigen eine signifikante Erhöhung der Geschwindigkeitskonstante, wie in der Abbildung 4.15 sichtbar ist. Die Wasserkonzentration hat dabei in allen Experimenten etwa $3 \%$ ausgemacht, was zunächst die Vermutung nahelegt, dass einzelne Wassermoleküle für den beobachteten Effekt verantwortlich sind. Die Berechnungen der Geschwindigkeitskonstante ohne Wasser haben die Bedeutung des Vorkomplexes zwischen $\mathrm{OH}$ und Acetaldehyd herausgestellt. Bei der Reaktion in Gegenwart von Wasser waren vor der Photolyse Acetaldehyd, Wasser und Wasserstoffperoxid im Badgas Stickstoff enthalten. Daher wurden auch Komplexe zwischen Wasser und Acetaldehyd gesucht, die analog zu den entsprechenden Strukturen mit dem OH-Radikal bei der Betrachtung ohne Wasser aufgebaut sind (Strukturen C und D in Abbildung 4.14). Durch Anlagerung eines OH-Radikals entsteht bei der Reaktion in Gegenwart von Wasser ein Vor- 
komplex E, der aus Acetaldehyd, $\mathrm{OH}$ und Wasser besteht. Durch seine ringförmige Struktur besitzt er zusätzliche Wasserstoffbrückenbindungen, die auch im entsprechenden Übergangszustand F vorhanden sind. Die Stabilisierungsenergie des Komplexes beträgt $44.6 \mathrm{~kJ} / \mathrm{mol}$, die Barrierenhöhe relativ zum Übergangszustand F der

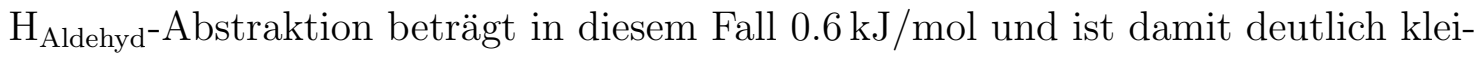
ner als die Barriere ohne Wasser. Die isomere Struktur, in der das Wassermolekül oberhalb der Carbonylgruppe positioniert ist, hat die gleiche Energiebarriere für die Reaktion wie in Abwesenheit von Wasser, so dass diese Struktur die Beschleunigung der Reaktion nicht erklären kann. In Abbildung 4.16 sind die Energieprofile der Reaktion mit Wasser dargestellt. Die geringere Barriere führt zu einer Verschiebung des Verzweigungsverhältnisses $Y$, vergleiche Gleichungen 4.2 und 4.4, zwischen den beiden Zerfallsmöglichkeiten des Komplexes hin zu einer nahezu einfangkontrollierten Reaktion, in der die Rückdissoziation des Komplexes zu den Edukten keine Rolle mehr spielt.

Eine solche Reaktion sollte eine geringe Temperaturabhängigkeit besitzen, was in den experimentellen Geschwindigkeitskonstanten in der Form nicht zu erkennen ist. Das ist auf die Art der Geschwindigkeitskonstante zurückzuführen, die experimentell zugänglich ist: Die Geschwindigkeitskonstante, die in Gegenwart von Wasser bestimmt wurde, ist das gewichtete Mittel der Konstante über den wasserfreien Reaktionspfad (Abbildung 4.13) und den Reaktionspfad unter Beteiligung von Wasser (Abbildung 4.16). Die Gleichgewichtskonstante der Wasserkomplexierung ist schließlich dafür verantwortlich, dass bei Raumtemperatur ein geringer Effekt zu beobachten ist, der mit sinkender Temperatur stetig wächst. Formal lässt sich diese Situation durch

$$
k_{\text {exp }}=\left(1-f_{\text {aggr }}\right) k_{0}+f_{\text {aggr }} k_{\text {aggr }}
$$

beschreiben. Dabei ist der Anteil der wasseraggregierten Reaktion mit $f_{\text {aggr }}$ gegeben. Die Geschwindigkeitskonstante für die nicht aggregierte Reaktion ist $k_{0}$, die der wasserkatalysierten $k_{\text {aggr }}$. Die experimentell beobachtete Geschwindigkeitskonstante wird mit $k_{\text {exp }}$ bezeichnet. 
Der bei dieser Reaktion gefundene katalytische Effekt von Wasser erhöht die bimolekulare Reaktionsgeschwindigkeit durch die Verringerung einer Energiebarriere bei der Anlagerung eines Wassermoleküls in einer spezifischen Konfiguration. Es handelt sich dabei um einen Effekt, der auf ein einzelnes Molekül Wasser in einem Übergangszustand zurückzuführen ist. Die Verringerung von Barrieren muss nicht zwangläufig zu einer erhöhten Geschwindigkeitskonstante führen, wenn die verbliebenen Barrieren oder die Dynamik der Reaktion dagegen sprechen [135]. Dennoch ist es wahrscheinlich, dass ein analoger katalytischer Effekt von Wassermolekülen nicht auf dieses System beschränkt ist.

Andere Systeme, die einen ähnlichen Effekt zeigen können, sollten über kleine Barrieren im Potential verfügen, um auf die Änderungen durch Wasserstoffbrückenbindungen empfindlich zu sein. Die Druckabhängigkeit sollte gut genug untersucht werden, um die Effekte des Schwingungsenergietransfers, die Wasser haben kann, beurteilen zu können. Eine mechanistische Aufklärung sollte neben Experimenten zur Bestimmung der Temperatur- und Druckabhängigkeit der Geschwindigkeitskonstante die Berechnung charakteristischer Punkte auf der Potentialfläche der Reaktion einbeziehen. Schließlich wäre eine Beurteilung der Abhängigkeit etwaiger Effekte durch Variation der Wasserkonzentration wünschenswert.

\subsection{Die Reaktion von $N, N$-Dimethylformamid mit $\mathrm{OH}$}

Die Reaktion von $N, N$-Dimethylformamid (DMF) und $\mathrm{OH}$ ist in analoger Weise zur Reaktion von Acetaldehyd und $\mathrm{OH}$ in Gegenwart von 3\% Wasserdampf untersucht worden. Die quantenmechanischen Rechnungen zur Bestimmung wichtiger Strukturen im Energieprofil der Reaktion sind noch nicht abgeschlossen. Die Anzahl veröffentlichter Daten bezüglich der Temperaturabhängigkeit und der Produkte ist deutlich geringer als bei Acetaldehyd.

Die Reaktion ist vorrangig wegen des Vergleichs zu der weniger polaren Carbonylfunktion von Acetaldehyd ausgewählt worden. Da DMF als Lösungsmittel in großtechnischen Verfahren verwendet wird, hat es trotz seines geringen Dampf- 


$\begin{array}{lcccc}\text { Düse } & T / \mathrm{K} & \rho_{\text {ges }} / 10^{17} \mathrm{~cm}^{-3} & k_{\mathrm{H}_{2} \mathrm{O}} / 10^{-11} \mathrm{~cm}^{3} \mathrm{~s}^{-1} & \text { Anzahl Messungen } \\ - & 295 & 2.6 & (1.6 \pm 0.08) & 3 \\ \text { M211E17 } & 118 & 1.1 & (5.8 \pm 0.87) & 3 \\ \text { M278E16 } & 106 & 0.75 & (5.4 \pm 0.81) & 6 \\ \text { M351E17 } & 77 & 0.85 & (11 \pm 1.7) & 9 \\ \text { M508E16 } & 60 & 0.65 & (15 \pm 1.3) & 6\end{array}$

Tabelle 4.6: Geschwindigkeitskonstanten und experimentelle Bedingungen für die Reaktion des OH-Radikals mit Acetaldehyd in Gegenwart von Wasserdampf: Die maximale Konzentration von Acetaldehyd in der Expansion betrug bei Raumtemperatur $9 \cdot 10^{14} \mathrm{~cm}^{-3}$ und bei den tiefen Temperaturen zwischen $7 \cdot 10^{14} \mathrm{~cm}^{-3}(118 \mathrm{~K}, 106 \mathrm{~K})$ und $2 \cdot 10^{14} \mathrm{~cm}^{-3}(77 \mathrm{~K}$, $60 \mathrm{~K})$.

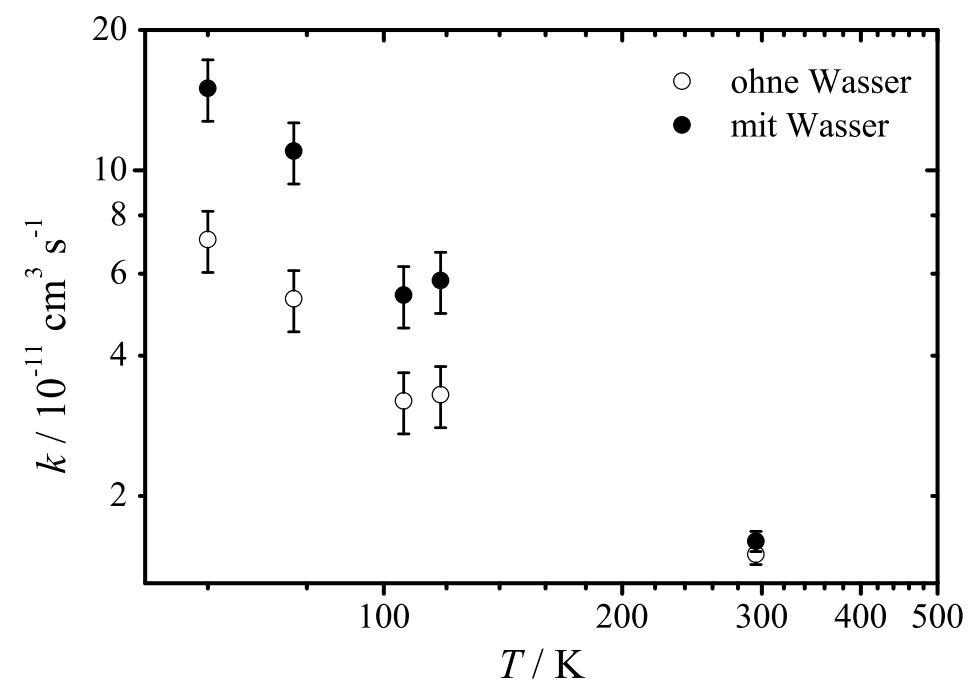

Abbildung 4.15: Der Vergleich zwischen der Geschwindigkeitskonstante der Reaktion von Acetaldehyd und $\mathrm{OH}$ mit und ohne Wasser zeigt den katalytischen Effekt, den Wassermoleküle auf die Reaktion haben. 


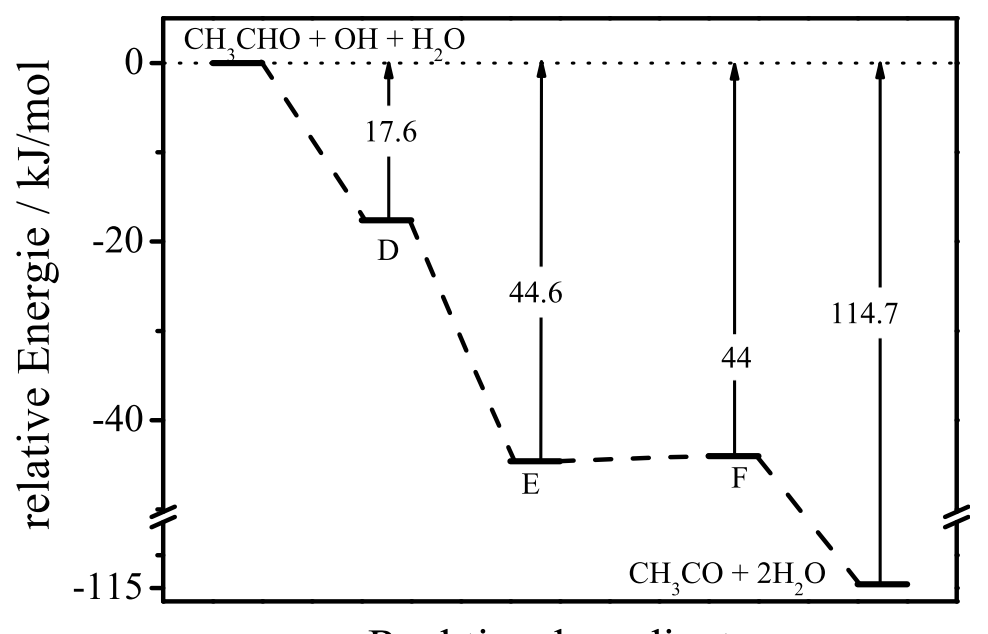

Reaktionskoordinate

Abbildung 4.16: Das Energieprofil der Reaktion zwischen Acetaldehyd und $\mathrm{OH}$ in Gegenwart von Wasser: Zunächst bildet sich ein Komplex D zwischen Acetaldehyd und Wasser, an diesen wird das OH-Radikal zu einer ringförmigen Struktur E angelagert. Der Übergangszustand zu den Produkten F ist gegenüber dem analogen Übergangszustand B der wasserfreien Reaktion stabilisiert. Die Energien sind die Ergebnisse von single-point Berechnungen mit $\operatorname{CCSD}(\mathrm{T})$ mit dem cc-pVTZ-Basissatz. 
drucks atmosphärenchemische Bedeutung. Dabei ist der hauptsächliche Abbau in der Troposphäre die Reaktion mit dem OH-Radikal. Der Mechanismus ist noch nicht detailliert bekannt, eine H-Abstraktion scheint der wahrscheinlichste Reaktionsweg zu sein [136, 137]. Diese Annahme wird durch Ergebnisse einer Studie der Reaktion von OH-Radikalen mit einer Reihe von Amiden in wässriger Lösung unterstützt, die eine H-Abstraktion an der Position CHO oder an den Methylgruppen des Stickstoffs nachweist [138]. Allerdings ist die direkte Übertragung der Produktverteilung auf die Reaktion in der Gasphase nicht zweifelsfrei gewährleistet. Mit steigender Substitution der Verbindungen mit aliphatischen Kohlenwasserstoffresten nahm die Reaktivität der Substituenten am Stickstoff zu. In der Arbeit von Koch et al. [137] ist eine negative Temperaturabhängigkeit für die Amide $N$-Methylacetamid, $N$ Methylpropamid, $N, N$-Dimethylacetamid und $N, N$-Dimethylpropamid für die Reaktion mit $\mathrm{OH}$ festgestellt worden. Dazu wurde die Geschwindigkeitskonstante bei $300 \mathrm{~K}$ und $384 \mathrm{~K}$ bestimmt.

Der zeitliche Verlauf der LIF-Intensität des OH-Radikals ist für Bedingungen pseudo-erster Ordnung in Abbildung 4.17 dargestellt. Die Ermittlung der Geschwindigkeitskonstante zweiter Ordnung ist für ein Einzelexperiment in Abbildung 4.18 gezeigt. Aus der Auftragung der Geschwindigkeitskonstanten gegen die Temperatur (siehe Abbildung 4.19) ist eine ausgeprägte negative Temperaturabhängigkeit abzulesen. Zu den niedrigsten Temperaturen nähert die Geschwindigkeitskonstante sich einem konstanten Wert. Die Übereinstimmung der Geschwindigkeitskonstante bei $300 \mathrm{~K}$ dieser Arbeit mit der von Solignac et al. ist gut. In Tabelle 4.7 sind die Geschwindigkeitskonstanten und experimentellen Bedingungen zusammengefasst.

Bisher stehen keine Produktstudien zur Verfügung, die Aussagen zum Mechanismus der Reaktion machen. Eine Additionsreaktion erscheint im Vergleich mit den Energieprofilen von Additionen von $\mathrm{OH}$ an Carbonylgruppen [5, 139] unwahrscheinlich. Ob der Ort der Wasserstoffabstraktion die Carbonylgruppe oder die Methylgruppen sind, lässt sich nicht mit Sicherheit sagen. Allein die Ergebnisse in wässriger Lösung weisen auf eine bevorzugte Abstraktion an den Methylgruppen hin. DMF ist ein polares Molekül, was die Bildung von Wasserstoffbrücken-gebundenen Komplexen wahrscheinlich macht. In vorläufigen Ergebnissen zur Berechnung relevanter Strukturen der Reaktion sind eine Reihe verschiedener Komplexe zwischen $\mathrm{OH}$ und 
DMF gefunden worden. Die bereits von Koch für andere Amide gefundene negative Temperaturabhängigkeit legt zusammen mit den Hinweisen auf schwach gebundene Komplexe einen Reaktionsmechanismus über einen Vorkomplex nahe, der für die Temperaturabhängigkeit verantwortlich sein kann.

Das Sättigungsverhalten der Geschwindigkeitskonstanten, dass bei den niedrigsten gemessenen Temperaturen auftritt, kann verschiedene Ursachen haben. Sollte sich durch eine Untersuchung der Reaktion mit ab initio-Methoden der VorkomplexMechanismus als wahrscheinlich herausstellen, kann das Erreichen einer einfanglimitierten Geschwindigkeitskonstante diese Sättigung erklären. Dazu könnte die Einfanggeschwindigkeitskonstante beispielsweise auf der Basis eines PST-Ansatzes berechnet werden, wie im Theorieteil im Abschnitt 2.2 erarbeitet. Die Korrektur für die Anisotropie der Wechselwirkung zwischen $\mathrm{OH}$ und DMF sollte dabei mit einem Dipol-Dipol Potential erfolgen. Damit ließe sich leicht zeigen, ob die Reaktion einfanglimitiert ist. Eine andere Erklärungsmöglichkeit ist der Einfluss der Kondensation von DMF. Der Konzentrationsbereich der Messungen wurde sorgfältig gewählt und die Auftragung der Geschwindigkeitskonstanten erster Ordnung gegen die Konzentration zeigt nur geringe Abweichungen von linearer Abhängigkeit. Dennoch kann die Bildung von DMF-Dimeren in geringem Ausmaß zu einer Verringerung der gemessenen Geschwindigkeitskonstanten beigetragen haben.

Die Experimente in der Gegenwart von Wasserdampf zeigen keinen signifikanten Unterschied in der Geschwindigkeitskonstante zu wasserfreien Bedingungen. Die Analogie der Carbonylfunktion zwischen Acetaldehyd und DMF spricht dennoch für einen solchen Effekt. Allerdings ist der Mechanismus der Reaktion zwischen $\mathrm{OH}$ und DMF noch nicht hinreichend genau untersucht, um den Ort der Abstraktion eines Wasserstoffatoms vorhersagen zu können. Wenn die Abstraktion eines Wasserstoffatoms an den Methylgruppen der bevorzugte Reaktionspfad ist, sind Übergangszustände in Analogie zur Reaktion von Acetaldehyd und $\mathrm{OH}$ nicht zu erwarten. Damit wäre auch kein Effekt auf die Geschwindigkeitskonstante zu beobachten.

Bei diesen Überlegungen sollte noch einmal darauf hingewiesen werden, dass es neben der Höhe von Barrieren noch weitere Einflüsse auf die Geschwindigkeitskonstante gibt, so dass sie trotz der Existenz günstiger Übergangszustände mit Wasser 
unverändert bleibt. Der Anteil der aggregierten Spezies $f_{\text {aggr }}$ spielt für die Beobachtung im Experiment eine entscheidende Rolle, wie aus Gleichung 4.5 ersichtlich.

$$
k_{\text {exp }}=\left(1-f_{\text {aggr }}\right) k_{0}+f_{\text {aggr }} k_{\text {aggr }}
$$

Der Unterschied zwischen der Geschwindigkeitskonstante der wasseraggregierten Reaktion $k_{\text {aggr }}$ und der wasserfreien Reaktion $k_{0}$ muss für einen experimentell beobachtbaren Effekt ebenfalls eine Mindestgröße haben. In Tabelle 4.8 sind die Geschwindigkeitskonstanten der Reaktion in Gegenwart von Wasserdampf mit wichtigen experimentellen Bedingungen aufgelistet.

Die laufenden quantenchemischen Untersuchungen werden einen großen Beitrag dazu leisten, den Mechanismus der Reaktion aufzuklären. Mit diesen Ergebnissen werden auch Aussagen über die Bedeutung von Wasseraggregaten für den Reaktionsmechanismus möglich sein. 


$\begin{array}{lcccc}\text { Düse } & T / \mathrm{K} & \rho_{\text {ges }} / 10^{17} \mathrm{~cm}^{-3} & k / 10^{-10} \mathrm{~cm}^{3} \mathrm{~s}^{-1} & \text { Anzahl Messungen } \\ - & 298 & 3.4 & (0.089 \pm 0.0064) & 5 \\ \text { M211E17 } & 117 & 0.9 & (2.64 \pm 0.35) & 4 \\ \text { M278E16 } & 111 & 0.80 & (2.50 \pm 0.38) & 5 \\ \text { M351E17 } & 80 & 0.90 & (2.59 \pm 0.51) & 6 \\ \text { M408E16 } & 72 & 0.95 & (1.92 \pm 0.14) & 4 \\ \text { M508E16 } & 63 & 0.71 & (1.24 \pm 0.44) & 6\end{array}$

Tabelle 4.7: Geschwindigkeitskonstanten und experimentelle Bedingungen für die Reaktion des OH-Radikals mit $N, N$-Dimethylformamid: Die maximale Konzentration von $N, N$-Dimethylformamid in der Expansion betrug bei Raumtemperatur $1.2 \cdot 10^{14} \mathrm{~cm}^{-3}$ und bei den tiefen Temperaturen zwischen $3 \cdot 10^{13} \mathrm{~cm}^{-3}$ (alle außer $\left.63 \mathrm{~K}\right)$ und $1.7 \cdot 10^{13} \mathrm{~cm}^{-3}(63 \mathrm{~K})$.

$\begin{array}{lcccc}\text { Düse } & T / \mathrm{K} & \rho_{\text {ges }} / 10^{17} \mathrm{~cm}^{-3} & k / 10^{-10} \mathrm{~cm}^{3} \mathrm{~s}^{-1} & \text { Anzahl Messungen } \\ - & 298 & 3.4 & (0.1029 \pm 0.0072) & 5 \\ \text { M211E17 } & 131 & 1.2 & (1.95 \pm 0.50) & 7 \\ \text { M278E16 } & 107 & 0.70 & (2.57 \pm 0.30) & 5 \\ \text { M351E17 } & 89 & 1.3 & (3.44 \pm 0.28) & 6 \\ \text { M408E16 } & 76 & 1.0 & (2.38 \pm 0.55) & 6 \\ \text { M508E16 } & 65 & 0.71 & (2.13 \pm 0.8) & 7\end{array}$

Tabelle 4.8: Geschwindigkeitskonstanten und experimentelle Bedingungen für die Reaktion des OH-Radikals mit $N, N$-Dimethylformamid in Gegenwart von Wasserdampf: Die maximale Konzentration von $N, N$ Dimethylformamid in der Expansion betrug bei Raumtemperatur 1.2 . $10^{14} \mathrm{~cm}^{-3}$ und bei den tiefen Temperaturen zwischen $3 \cdot 10^{13} \mathrm{~cm}^{-3}$ (alle außer $65 \mathrm{~K})$ und $1.7 \cdot 10^{13} \mathrm{~cm}^{-3}(65 \mathrm{~K})$. 


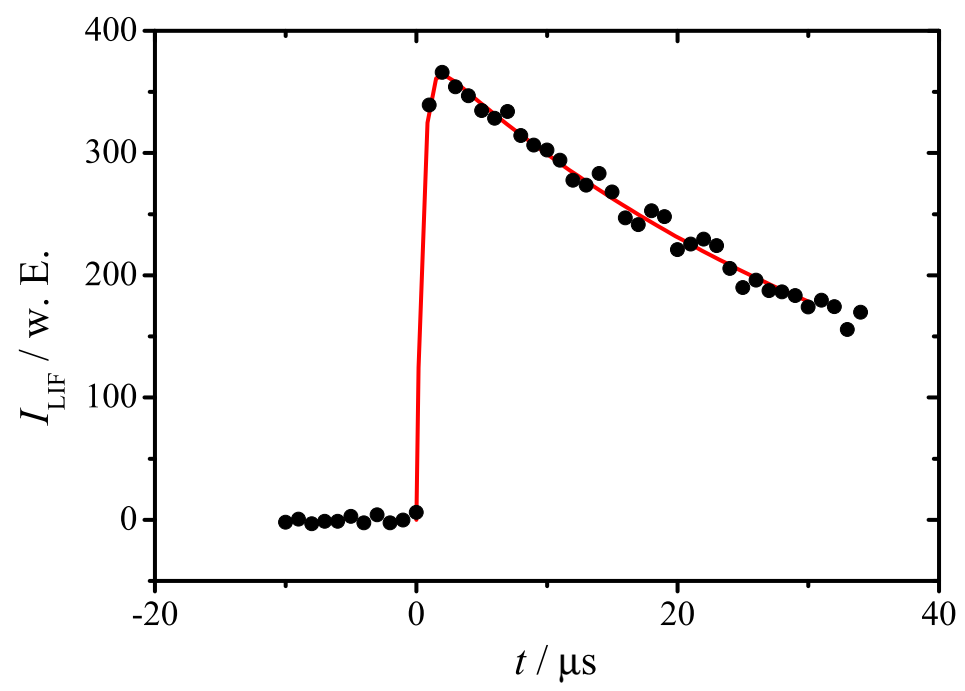

Abbildung 4.17: Das Fluoreszenzsignal von $\mathrm{OH}$ zeigt eine Zeitabhängigkeit pseudoerster Ordnung, hier dargestellt für $72 \mathrm{~K}$, eine Gesamtdichte von $0.95 \cdot 10^{17} \mathrm{~cm}^{-3}$ und eine Konzentration von $2.44 \cdot 10^{13} \mathrm{~cm}^{-3}$ an $N, N$-Dimethylformamid dargestellt.

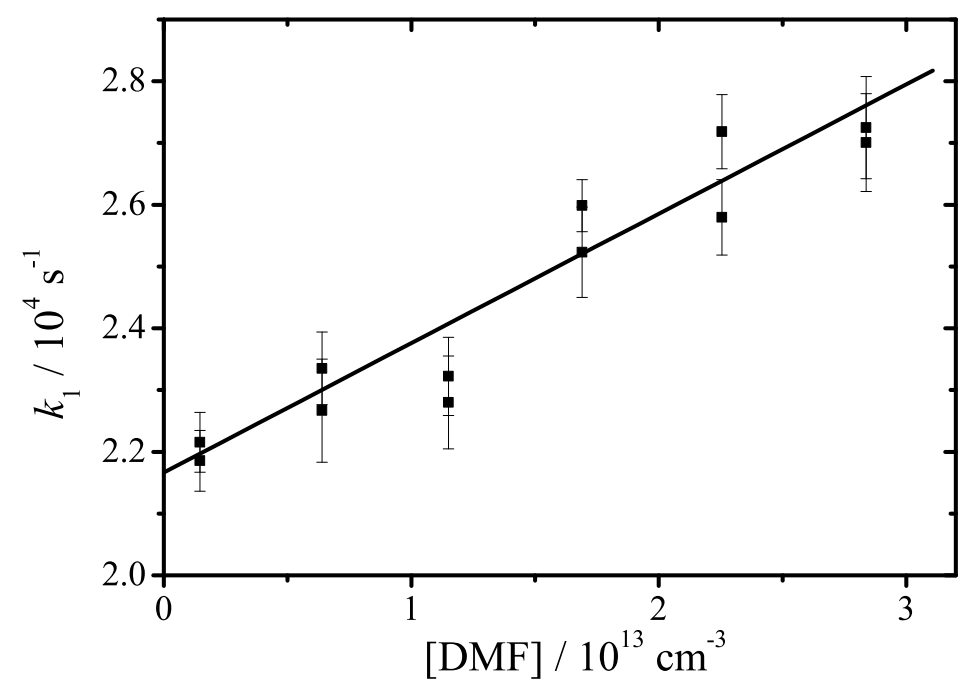

Abbildung 4.18: Die Geschwindigkeitskonstanten pseudo-erster Ordnung werden gegen die Konzentration von DMF aufgetragen, in dieser Abbildung für $72 \mathrm{~K}$ und eine Gesamtdichte von $0.95 \cdot 10^{17} \mathrm{~cm}^{-3}$. Die Geschwindigkeitskonstante dieser Einzelmessung wird aus der angepassten Gerade zu $2.05 \cdot 10^{-10} \mathrm{~cm}^{3} \mathrm{~s}^{-1}$ bestimmt. 


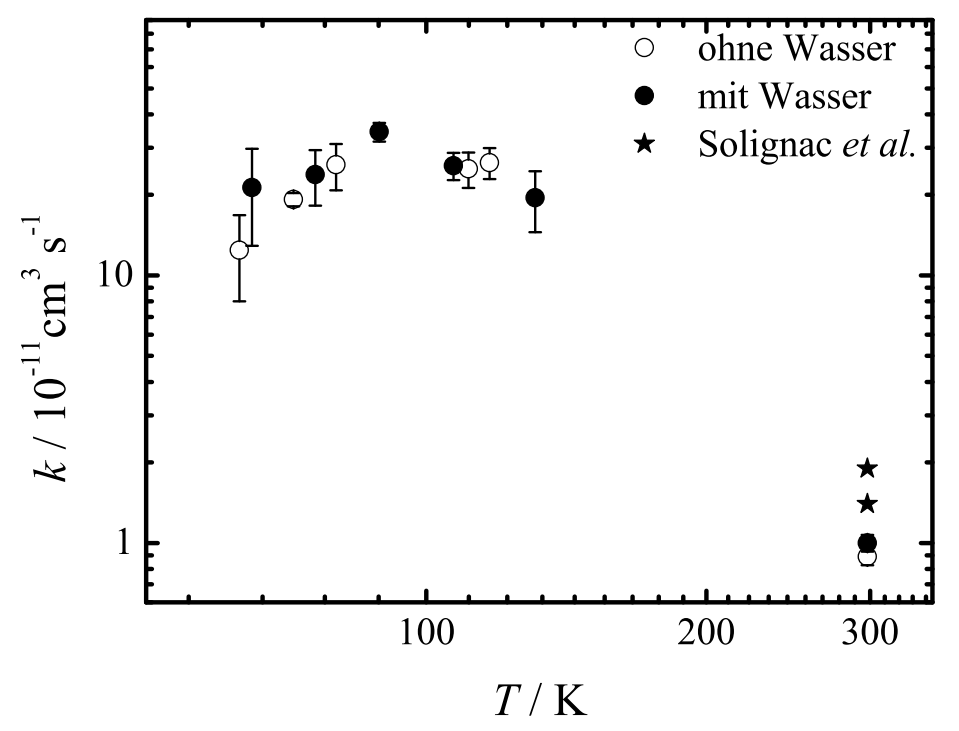

Abbildung 4.19: Die temperaturabhängige Geschwindigkeitskonstante der Reaktion von $\mathrm{OH}$ mit $N, N$-Dimethylformamid mit der Geschwindigkeitskonstante von Solignac et al. [136]. 


\section{Kapitel 5}

\section{Ausblick}

Aktuell wird der experimentelle Aufbau um einen Hochdruckteil erweitert, der die Expansion von überkritischem Kohlendioxid in das Stagnationsvolumen ermöglicht. Im Zuge dieser Erweiterung wird zudem die spektroskopische Bestimmung der Reaktandenkonzentration in der Zuleitung ermöglicht. Die ersten Messungen sollen mit Menthol als Reaktionspartner des OH-Radikals durchgeführt werden. Durch die Lösungseigenschaften der überkritischen Phase sind Substanzen zur Messung zugänglich, die sonst nur durch eine starke Erwärmung der Düse und des Stagnationsvolumens messbar wären, wie in der Arbeitsgruppe von Rowe gezeigt [36, 57]. Die Erwärmung bringt einige Nachteile mit sich, die mit der Verwendung überkritischer Lösungen umgangen werden können.

Im Rahmen der Zusammenarbeit mit der Arbeitsgruppe Suhm im Graduiertenkolleg 782 werden Messungen mit Hilfe FTIR-Technik durchgeführt. Das Ziel der Experimente ist die Messung der Clustergrößenverteilung bei verschiedenen Abständen zum Ausgang der Lavaldüse. Die Änderung der Größenverteilung kann Aufschluss über die Bildungsgeschwindigkeitskonstanten unter konstanten Temperatur- und Dichtebedingungen geben.

Die Lavaldüse kann nicht nur als chemischer Reaktor eingesetzt werden, wie an anderer Stelle bereits betont. Ein neues Einsatzgebiet könnte ihre Verwendung in der Füllung von Molekülfallen sein, wie sie die Arbeitsgruppe um Meijer beschreibt. Eine mögliche Zusammenarbeit ist bereits diskutiert und als aussichtsreich beurteilt worden. 
Die Reaktion von $\mathrm{OH}$ und NO, die im Hinblick auf den Einfluss von Wasserclustern auf die Geschwindigkeitskonstante interessant ist, wird in nächster Zeit untersucht werden. Aktuell werden in anderen Gruppen theoretische Untersuchungen dieser Reaktion angestellt.

Neben den bereits konkret geplanten oder anlaufenden Projekten wird die Apparatur möglicherweise zur Untersuchung heterogener Reaktionen verwendet werden, die jüngeren Entwicklungen in der Erforschung der Atmosphärenchemie lassen darauf schließen, dass Aerosolen und kleinen Partikeln in der Chemie der Atmosphäre eine weitreichende Bedeutung zukommt.

Abhängig von den zu untersuchenden Stoffen und Reaktionen kann es nötig werden, den Temperaturbereich oder die Detektionsmethode zu verändern beziehungsweise zu erweitern. In der Vergangenheit wurde dabei die Verwendung eines Massenspektrometers diskutiert. Sollten die Experimente mit der Lavaldüse als Füllstrahl für eine Molekularstrahlapparatur erfolgreich sein, könnten die dort gewonnenen Erfahrungen auf das Problem der Einkopplung des Lavaldüsenstrahls in ein Massenspektrometer übertragen werden, was diese Technik mit einer dadurch verkürzten Umbauzeit wieder attraktiv macht. 


\section{Abbildungsverzeichnis}

2.1 Effektive Potentiale verschiedener Übergangszustände . . . . . . . . 20

2.2 Drehimpuls- und Energieerhaltung bei zentralem Potential . . . . . 23

2.3 Die verschiedenen schematisierten Fälle der Eingangs- und Ausgangskanäle einer komplexbildenden bimolekularen Reaktion. . . . . . . . 31

2.4 rotational channel switching . . . . . . . . . . . . . . . . . . . . 34

2.5 vibrational channel switching . . . . . . . . . . . . . . . 34

2.6 Schematische Darstellung der KCSF-Methode . . . . . . . . . . . . 38

3.1 Der divergente Teil einer achsensymmetrischen Lavaldüse . . . . . . 45

3.2 Illustration eines Prandtl-Meyer-Expansionsfächers . . . . . . . . . 47

3.3 Dreidimensionale Darstellung des Aufpralldrucks . . . . . . . . . . . 53

3.4 Messungen der Temperatur im Strahl der Lavaldüse durch ein PitotRohr für die Düse M358E16 . . . . . . . . . . . . . . . . 53

3.5 Ein schematisierter Überblick über den experimentellen Aufbau der Lavalapparatur . . . . . . . . . . . . . . . . 56

3.6 Das Stagnationsvolumen mit den Magnetventilen V1, V2, dem Drucksensor für $p_{0}$ und der Spülung für das Fenster, rechts im Bild . . . . 58

3.7 Typisches Zeitprofil der OH Fluoreszenz . . . . . . . . . . . . . . . 62

3.8 Auftragung der $k_{1}$ gegen die Konzentration des Reaktionspartners . 62

4.1 KCSF Signale für $266.8 \mathrm{~nm} \ldots \ldots \ldots$. . . . . . . . . . . 67

$4.2 \mathrm{KCSF}$ Signale für $267.5 \mathrm{~nm}$. . . . . . . . . . . . . . . . . . 68

4.3 Auftragung von $k$ gegen $T$ für Acetylen . . . . . . . . . . . . . . . . 73

4.4 Auftragung von $k$ gegen $T$ für Ethen . . . . . . . . . . . . . 76

4.5 Auftragung von $k$ gegen $T$ für Benzol . . . . . . . . . . . . . . 78 
4.6 Schematisierte Reaktionskoordinate der Reaktion von Alaninethylester und $\mathrm{OH} \ldots \ldots \ldots$. . . . . . . . . . . . . . 81

4.7 Ermittlung der Geschwindigkeitskonstante pseudo-erster Ordnung für Alaninethylester . . . . . . . . . . . . . . . . . . . 81

4.8 Ermittlung der Geschwindigkeitskonstante zweiter Ordnung für Alaninethylester . . . . . . . . . . . . . . . . 82

4.9 Auftragung von $k$ gegen $T$ für Alaninethylester . . . . . . . . . . . 82

4.10 Ermittlung der Geschwindigkeitskonstante pseudo-erster Ordnung für Acetaldehyd . . . . . . . . . . . . . . . . . 87

4.11 Ermittlung der Geschwindigkeitskonstante zweiter Ordnung für Alaninethylester . . . . . . . . . . . . . . . 87

4.12 Die temperaturabhängige Geschwindigkeitskonstante der Reaktion von Acetaldehyd und $\mathrm{OH}$. . . . . . . . . . . . . . . . . 88

4.13 Das Energieprofil der Reaktion von Acetaldehyd und $\mathrm{OH}$. . . . . . 89

4.14 ab initio Strukturen von Acetaldehyd, OH und Wasser . . . . . . . 91

4.15 Vergleich der Geschwindigkeitskonstante von Acetaldehyd und $\mathrm{OH}$ mit und ohne Wasser . . . . . . . . . . . . . . . . . . . 95

4.16 Das Energieprofil der Reaktion zwischen Acetaldehyd und $\mathrm{OH}$ in Gegenwart von Wasser . . . . . . . . . . . . . . 96

4.17 Ermittlung der Geschwindigkeitskonstante pseudo-erster Ordnung für

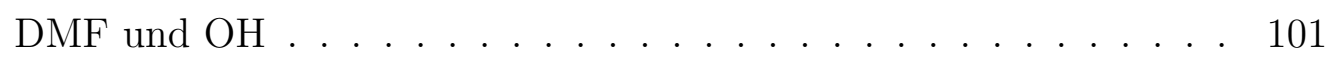

4.18 Ermittlung der Geschwindigkeitskonstante zweiter Ordnung für DMF

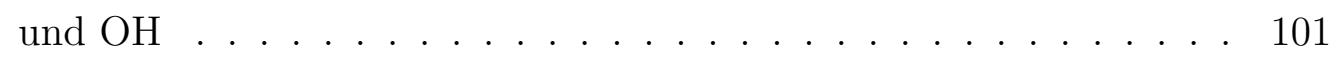

4.19 Die temperaturabhängige Geschwindigkeitskonstante der Reaktion

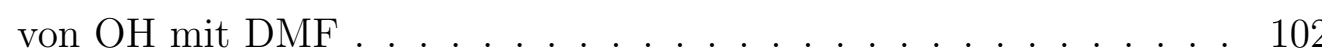




\section{Literaturverzeichnis}

[1] E. Herbst, Ann. Rev. Phys. Chem., 1995, 46, 27.

[2] B. Abel, Zustands-, Energie-, und Drehimpulsabhängigkeit der Dynamik kleiner Moleküle, Habilitationsschrift, Georg-August Universität, 1999.

[3] S. Arrhenius, Z. Phys. Chem., 1889, 4, 226.

[4] B. J. Finlayson-Pitts und J. Pitts, J. N., Chemistry of the Upper and Lower Atmosphere, Academic Press, San Diego London, 2000.

[5] M. Cameron, V. Sivakumaran, T. J. Dillon und J. N. Crowley, Phys. Chem. Chem. Phys., 2002, 4, 3628-3638.

[6] B. D’Anna, V. Bakken, J. A. Beukes, C. J. Nielsen, K. Brudnik und J. T. Jodkowski, Phys. Chem. Chem. Phys., 2003, 5, 1790-1805.

[7] G. S. Tyndall, J. J. Orlando, T. J. Wallington, M. D. Hurley, M. Goto und M. Kawasaki, Phys. Chem. Chem. Phys., 2002, 4, 2189-2193.

[8] S. Aloisio und J. S. Francisco, Acc. Chem. Res., 2000, 33, 825-830.

[9] G. Frost und V. Vaida, J. Geophys. Res., 1995, 100, 18803.

[10] V. Vaida, H. G. Kjaergaard, P. E. Hintze und D. J. Donaldson, science, 2003, 299, 1566.

[11] K. Tao, F. M.and Higgins, W. Klemperer und D. D. Nelson, Geophys. Res. Lett., 1996, 23, 1797.

[12] J. C. Hansen und J. S. Francisco, Chem. Phys. Chem., 2002, 3 (10), 833. 
[13] H. Fliegl, A. Glöß, O. Welz, M. Olzmann und W. Klopper, J. Chem. Phys., 2006, 125, 054312 .

[14] J. Jayne, U. Pöschl, Y. Chen, D. Dai, L. Molina, C. Worsnop, C. Kolb und M. Molina, J. Phys. Chem. A, 1997, 101, 10000.

[15] T. Finkel und N. J. Holbrook, Nature, 2000, 408, 239.

[16] A. Galano, J. R. Alvarez-Idaboy, L. A. Montero und A. Vivier-Bunge, J. Comp. Chem., 2001, 22, 1138-1153.

[17] S. Hamon, S. De Picard, A. Canosa und B. R. Rowe, J. Chem. Phys., 2000, $112(10), 4506-4516$.

[18] I. W. M. Smith, Angew. Chem. Int. Ed., 2006, 45, $2842-2861$.

[19] I. R. Sims und I. W. M. Smith, Ann. Rev. Phys. Chem., 1995, 46, 109-137.

[20] B. R. Rowe, G. Dupeyrat, J. B. Marquette und P. Gaucherel, J. Chem. Phys., 1984, 80 (10), 4915-4921.

[21] B. R. Rowe und J. B. Marquette, Int. J. Mass Spectrom. Ion Proc., 1987, 80, 239-254.

[22] D. B. Atkinson und M. A. Smith, J. Phys. Chem., 1994, 98, 5797.

[23] C. Mullen und M. A. Smith, Journal of Physical Chemistry A, 2005, 109(17), 3893-3902.

[24] R. Hoobler, B. Opansky und S. Leone, J. Phys. Chem. A, 1997, 101 (7), 1338.

[25] F. Goulay und S. R. Leone, Journal of Physical Chemistry A, 2006, 110(5), 1875-1880.

[26] T. Spangenberg, S. Köhler, B. Hansmann, U. Wachsmuth und B. Abel, J. Phys. Chem. A, 2004, 108, 7527-7534. 
[27] E. Vöhringer-Martinez, B. Hansmann, H. Hernandez, J. S. Francisco, J. Troe und B. Abel, Science, 2007, 315, 497.

[28] B. Hansmann und B. Abel, ChemPhysChem, 2007, 8, 343-356.

[29] N. Daugey, P. Caubet, B. Retail, M. Costes, A. Bergeat und G. Dorthe, Physical Chemistry Chemical Physics, 2005, 7(15), 2921-2927.

[30] I. R. Sims, J. H. Queffelec, D. Travers, B. R. Rowe, L. Herbert, J. Karthäuser und I. W. M. Smith, Chem. Phys. Lett., 1993, 211, 461.

[31] I. R. Sims, J. L. Queffelec, A. Defrance, C. Rebrion-Rowe, D. Travers, P. Bocherel, B. R. Rowe und I. W. M. Smith, J. Chem. Phys., 1994, 100 (6), 4229-4241.

[32] D. Carty, V. Le Page, I. R. Sims und I. W. M. Smith, Chem. Phys. Lett., 2001, 344, 310-316.

[33] I. R. Sims und I. W. M. Smith, Chem. Phys. Lett., 1988, 151 (6), 481.

[34] T. Spangenberg, Kinetik der Reaktionen des Hydroxyradikals mit ungesättigten Kohlenwasserstoffen in einer Lavaldüsenexpansion, Doktorarbeit, GeorgAugust Universität Göttingen, 2003.

[35] A. B. Vakhtin, J. A. Murphy und S. R. Leone, J. Phys. Chem. A, 2003, 107, 10055 .

[36] F. Goulay, C. Rebrion-Rowe, L. G. J. L., S. D. Le Picard, A. Canosa und B. R. Rowe, Journal of Chemical Physics, 2006, 122, 104 308/1-104308/7.

[37] D. Carty, A. Goddard, S. P. K. Koehler, I. R. Sims und I. W. M. Smith, Journal of Physical Chemistry A, 2006, 110(9), 3101-3109.

[38] P. Sharkey, I. R. Sims, I. W. M. Smith, P. Bocherel und B. R. Rowe, J. Chem. Soc. Faraday Trans., 1994, 90, 3609.

[39] D. Chastaing, P. L. James und I. W. M. Smith, Faraday Discuss., 1998, 109, 165. 
[40] S. Lee und S. R. Leone, Chem. Phys. Lett., 2000, 329 (5-6), 443.

[41] A. Canosa, I. R. Sims, D. Travers, I. W. M. Smith und B. R. Rowe, Astron. Astrophys., 1997, 323, 644-651.

[42] P. Bocherel, L. B. Herbert, B. R. Rowe, I. R. Sims, I. W. M. Smith und D. Travers, J. Phys. Chem., 1996, 100 (8), 3063-3069.

[43] R. A. Brownsworth, A. Canosa, B. R. Rowe, I. R. Sims, I. W. M. Smith, D. W. A. Stewart, A. C. Symonds und D. Travers, J. Chem. Phys., 1997, 106 (18), 7662-7677.

[44] R. A. Brownsword, L. B. Herberg, I. W. M. Smith und D. W. A. Stewart, Journal of the Chemical Society, Faraday Transactions, 1996, 92(7), 1087-94.

[45] S. D. Le Picard, A. Canosa, B. R. Rowe, R. A. Brownsword und I. W. M. Smith, Journal of the Chemical Society, Faraday Transactions, 1998, 94(19), 2889-2893.

[46] D. Chastaing, S. D. Le Picard, I. R. Sims und I. W. M. Smith, Astronomy and Astrophysics, 2001, 365(2), 241-247.

[47] D. Chastaing, P. L. James, I. R. Sims und I. W. M. Smith, Phys, Chem. Chem. Phys., 1999, 1 (9), 2247.

[48] D. Chastaing, S. D. Le Picard und I. R. Sims, Journal of Chemical Physics, 2000, 112(19), 8466-8469.

[49] S. D. Le Picard, A. Canosa, D. Travers, D. Chastaing, B. R. Rowe und T. Stoecklin, Journal of Physical Chemistry A, 1997, 101(51), 9988-9992.

[50] A. Canosa, S. D. Le Picard, S. Gougeon, C. Rebrion-Rowe, D. Travers und B. R. Rowe, Journal of Chemical Physics, 2001, 115(14), 6495-6503.

[51] S. D. Le Picard, A. Canosa, D. Reignier und T. Stoecklin, Physical Chemistry Chemical Physics, 2002, 4, 3659-3664. 
[52] W. D. Geppert, F. Goulay, C. Naulin, M. Costes, A. Canosa, S. D. Le Picard und B. R. Rowe, Physical Chemistry Chemical Physics, 2005, 6(3), 566-571.

[53] A. Canosa, S. D. Le Picard und W. D. Geppert, Journal of Physical Chemistry A, 2004, 108(29), 6183-6185.

[54] S. D. Le Picard, A. Canosa, W. Geppert und T. Stoecklin, Chem. Phys. Lett., 2004, $385(5,6), 502-506$.

[55] S. M. A. Wright, I. R. Sims und I. W. M. Smith, J. Phys. Chem. A, 2000, 104, $10347-10355$.

[56] A. Bonnamy, R. Georges, A. Benidar, J. Boissoles, A. Canosa und B. R. Rowe, J. Chem. Phys., 2003, 118 (8), 3612-3621.

[57] F. Goulay, C. Rebrion-Rowe, L. Biennier, S. D. Le Picard, A. Canosa und B. R. Rowe, Journal of Physical Chemistry A, 2006, 110, 3132-3137.

[58] J. B. Marquette, B. R. Rowe, G. Dupeyrat und E. Roueff, Astronomy and Astrophysics, 1985, 147 (1), 115-20.

[59] S. Y. T. van de Meerakker, P. H. M. Smeets, N. Vanhaecke, R. T. Jongma und G. Meijer, Physical Review Letters, 2005, 94, 23004.

[60] F. Crompvoets, H. Bethlem, R. Jongma und G. Meijer, Nature, 2001, 411, $174-176$.

[61] H. Frerichs, T. Lenzer, K. Luther und D. Schwarzer, Phys. Chem. Chem. Phys., 2005, 7, 620.

[62] T. Lenzer, K. Luther, K. Reihs und A. C. Symonds, J. Chem. Phys., 2000, 112 (9), 4090-4110.

[63] H. G. Löhmansröben und K. Luther, Chem. Phys. Lett., 1988, 144 (5,6), 473.

[64] U. Hold, T. Lenzer, K. Luther, K. Reihs und A. C. Symonds, J. Chem. Phys., 2000, 112 (9), 4076-4089. 
[65] O. K. Rice und H. C. Ramsperger, J. A. Che. Soc., 1927, 49, 1617.

[66] L. S. Kassel, J. Phys. Chem., 1928, 32, 1065.

[67] R. A. Marcus und O. K. Rice, J. Phys. Chem., 1952, 55, 894.

[68] R. A. Marcus, J. Chem. Phys., 1966, 45, 4500.

[69] W. Forst, Unimolecular reactions, Cambridge University Press, 2003.

[70] R. Gilbert und S. Smith, Theory of Unimolecular and Recombination Reactions, Blackwell Scientific Publications, Oxford, 1990.

[71] M. Quack und J. Troe, Ber. Bunsenges. Phys. Chem., 1974, 78, 240-252.

[72] M. Quack und J. Troe, Ber. Bunsenges. Phys. Chem., 1975, 79, 170-183.

[73] J. Troe, J. Chem. Phys., 1983, 79, 6017.

[74] G. Worry und R. A. Marcus, J. Chem. Phys., 1977, 67, 1636.

[75] E. E. Nikitin, Theor. Exp. Chem., 1965, 1, 83, 90.

[76] P. Pechukas und J. C. Light, J. Chem. Phys., 1965, 42, 3281.

[77] E. E. Nikitin, Theor. Exp. Chem., 1965, 1, 275.

[78] W. Forst, Phys. Chem. Chem. Phys., 1999, 1, 1283.

[79] L. Bass, W. L. Chesnavich und M. T. Bowers, J. Am. Chem. Soc., 1979, 101, 5493.

[80] D. C. Clary, Ann. Rev. Phys. Chem., 1990, 41, 61.

[81] D. C. Clary, E. Buonomo, I. R. Sims, I. W. M. Smith, W. D. Geppert, C. Naulin, M. Costes, L. Cartechini und P. Casavecchia, Journal of Physical Chemistry A, 2002, 106(23), 5541-5552.

[82] J. Troe, Chem. Phys. Lett, 1985, 122, 425. 
[83] J. Troe und V. G. Ushakov, J. Phys. Chem. A, 2006, 110, 6732-6741.

[84] D. C. Clary und J. P. Henshaw, Faraday Discuss. Chem. Soc., 1987, 84, 333-49.

[85] A. I. Maergoiz, E. E. Nikitin, J. Troe und V. G. Ushakov, J. Chem. Phys., 2002, 11\%, 4201 .

[86] G. H. Dieke und H. M. Crosswhite, J. Quant. Spectrosc. Radiat. Transfer, 1962, 2, 97-199.

[87] A. B. Vakhtin, S. Lee, D. E. Heard, I. W. M. Smith und S. R. Leone, J. Phys. Chem., 2001, 105, 7889-7895.

[88] I. R. Sims, W. M. Smith, P. Bocherel, A. Defrance, D. Travers und B. R. Rowe, Journal of the Chemical Society, Faraday Transactions, 1994, 90(11), 1473-8.

[89] D. Fulle, H. F. Hamann, H. F. Hippler und J. Troe, J. Chem. Phys., 1996, 105, 983.

[90] R. Forster, M. Frost, D. Fulle, H. F. Hamann, H. Hippler, A. Schlepegrell und J. Troe, J. Chem. Phys., 1995, 103, 2949.

[91] J. Troe, J. Chem. Soc. Faraday Trans., 1994, 90, 2303-2317.

[92] J. A. Miller und C. F. Melius, in "Symp. (Int.) Combust.", Bd. 22, 1988 1031-1039, 1031-1039, inproceedings.

[93] J. P. Senosiain, S. J. Klippenstein und J. A. Miller, J. Phys. Chem. A, 2006, 110, 6960-6970.

[94] E. E. Greenwald, S. W. North, Y. Georgievskii und S. J. Klippenstein, J. Phys. Chem. A, 2005, 109, 6031-6044.

[95] B. Abel, N. Lange, F. Reiche und J. Troe, J. Chem. Phys., 1999, 110, 1404. 
[96] J. J. Klaassen, S. L. Coy, J. I. Steinfeld und B. Abel, J. Chem. Phys., 1994, 101, 10533.

[97] B. Abel, B. Herzog, H. Hippler und J. Troe, J. Chem. Phys., 1989, 91, 900.

[98] B. Abel, H. Hippler und J. Troe, J. Chem. Phys., 1992, 96, 8872.

[99] J. D. Cox und G. Pilcher, Thermochemistry of organic and organometallic compounds, Academic Press, London, 1970.

[100] K. Reihs, Besetzungsänderungen im elektronischen Grundzustand während der Stoßrelaxation von hoch schwingungsangeregtem Toluol, Dissertation, GeorgAugust Universität, 1989.

[101] H. Hippler, J. Troe und H. J. Wendelken, J. Chem. Phys., 1983, 78 (11), 6709-6717.

[102] J. Troe, J. Chem. Phys., 1982, 77 (7), 3485-3492.

[103] D. B. Atkinson, Ph.d. thesis, University of Arizona, 1995.

[104] D. B. Atkinson und M. A. Smith, Rev. Sci. Instrum., 1995, 66 (9), 4434.

[105] B. Hansmann, Die Reaktionen des Hydroxylradikals mit Propen und Isopren bei tiefen Temperaturen, Diplomarbeit, Georg-August-Universität Göttingen, 2003.

[106] W. C. Moger und D. B. Ramsay, Techn. Ber. AEDC-TDR-64-110, Arnold Engineering Development Center, 1964, techreport.

[107] M. Ließmann, Kinetik von Aminosäureestern in Lavaldüsenexpansionen, Diplomarbeit, Georg August Universität Göttingen, 2006.

[108] B. M. Toselli, J. D. Brenner, M. L. Yerram und W. E. Chin, J. Chem. Phys., 1991, 95 (1), 176-188.

[109] R. Karunanandan, Die Reaktion von Benzol mit dem Hydroxylradikal im kalten Düsenstrahl einer Lavaldüse, Diplomarbeit, Georg August Universität Göttingen, 2004. 
[110] M. D. Marshall, J. B. Davey, M. E. Greenslade und M. I. Lester, Journal of Chemical Physics, 2004, 121 (12), 5845-5851.

[111] J. R. Alvarez-Idaboy, N. Mora-Diez und A. Vivier-Bunge, J. Am. Chem. Soc., 2000, 122 (15), 3715-3720.

[112] I. Diaz-Costa, R. Alvarez-Idaboy und A. Vivier-Bunge, Int. J. Chem. Kinet., 1999, 31 (1), 29 .

[113] N. M. Donahue, Chem. Rev., 2003, 103, 4593-4604.

[114] A. Liu, W. A. Mulac und C. D. Jonah, J. Phys. Chem., 1988, 92 (21), 59425945.

[115] D. Fulle, H. F. Hamann, H. Hippler und C. P. Jänsch, Ber. Bunsenges. Phys. Chem., 1997, 101 (10), 1433-1442.

[116] C. Sosa und H. B. Schlegel, J. Am. Chem. Soc., 1987, 109 (14), 4193-4198.

[117] D. L. Singleton und R. J. Cvetanovic, J. Am. Chem. Soc., 1976, 98, 68126819.

[118] C. Barckholtz, T. A. Barckholtz und C. M. Hadad, J. Phys. Chem. A, 2001, 105 (1), 140-152.

[119] R. H. Schuler und G. Albarran, Radiation Physics and Chemistry, 2002, 64 (3), 189-195.

[120] F. P. Tully, A. R. Ravishankara, R. L. Thompson, J. M. Nicovich, R. C. Shah, N. M. Kreutter und P. H. Wine, J. Phys. Chem., 1981, 85, 2262-2269.

[121] S. Madronich und W. Felder, J. Phys. Chem., 1985, 89, 3556-3561.

[122] T. J. Wallington, D. M. Neumann und M. J. Kurylo, Int. J. Chem. Kinet., 1987, 19, 725-739.

[123] S. C. Lin, T. C. Kuo und Y. P. Lee, Rad. Phys. and Chem., 2002, 64, 189-195. 
[124] B. N. Nukuna, M. B. Goshe und V. E. Anderson, J. Am. Chem. Soc., 2001, 123, 1208- 1214.

[125] I. Stefanic, M. Bonifacic, K.-D. Asmus und D. A. Armstrong, J. Phys. Chem. A, 2001, 105, 8681-8690.

[126] M. Ließmann, B. Hansmann, B. Abel und J. S. Francisco, "Reaction of OH with Alaninethylester, low temperature rate constants and ab initio calculations", Unpublished results.

[127] S. Hesse, FTIR-Spektroskopie von kleinen Aminosäure- und Peptidmodellen in der Gasphase, Diplomarbeit, Georg-August-Universität Göttingen, 2005.

[128] HTTP://www.sciencemag.org/cgi/content/full/315/5811/497/DC1.

[129] E. Vöhringer-Martinez, Reaktionen des OH-Radikals in Lavaldüsenexpansionen in Gegenwart von Wasser, Diplomarbeit, Georg-August-Universität Göttingen, 2004.

[130] P. H. Taylor, T. Yamada und P. Marshall, Int. J. Chem. Kinet., 2006, 38, 489-495.

[131] J. R. Alvarez-Idaboy, N. Mora-Diez, R. J. Boyd und A. Vivier-Bunge, J. Am. Chem. Soc., 2001, 123, 2018-2024.

[132] J. V. Michael, D. G. Keil und K. R. B., J. Chem. Phys., 1985, 83, 1630.

[133] V. Sivakumaran und J. N. Crowley, Phys. Chem. Chem. Phys., 2003, 5, 106.

[134] M. J. Frisch, G. W. Trucks, H. B. Schlegel, G. E. Scuseria, M. A. Robb, J. R. Cheeseman, V. G. Zakrzewski, J. J. A. Montgomery, R. E. Stratmann, J. C. Burant, S. Dapprich, J. M. Millam, A. D. Daniels, K. N. Kudin, M. C. Strain, O. Farkas, J. Tomasi, V. Barone, M. Cossi, R. Cammi, B. Mennucci, C. Pomelli, C. Adamo, S. Clifford, J. Ochterski, G. A. Peterson, P. Y. Ayala, Q. Cui, K. Morokuma, D. K. Malick, A. D. Rabuck, K. Raghavachari, J. B. Foresman, J. Cioslowski, J. V. Ortiz, B. B. Stefanov, G. Liu, A. Liashenko, P. Piskorz, I. Komaromi, R. Gomperts, R. L. Martin, D. J. Fox, T. Keith, 
M. A. Al-Laham, C. Y. Peng, A. Nanayakkara, C. Gonzales, M. Challacombe, P. M. W. Gill, B. Johnson, W. Chen, M. W. Wong, J. L. Andres, C. Gonzales, M. Head-Gordon, E. S. Replogle und J. A. Pople, "Gaussian 98, Revision A.5", 1998.

[135] S. Canneaux, N. Sokolowski-Gomez, E. Henon, F. Bohr und S. Dobe, Phys. Chem. Chem. Phys., 2004, 6, 5172.

[136] G. Solignac, A. Mellouki, G. Le Bras, I. Barnes und T. Benter, J. Photochem. Photobiol. A, 2005, 176, 136-142.

[137] R. Koch, W. U. Palm und C. Zetzsch, Int. J. Chem. Kinet., 1997, 29, 81-87.

[138] E. Hayon, T. N. Ibata, N. Lichtin und M. Simic, J. Am. Chem. Soc., 1970, 92, 3898.

[139] V. Sivakumaran, D. Höschler, T. J. Dillon und J. N. Crowley, Phys. Chem. Chem. Phys., 2003, 5, 4821-4827. 



\section{Danksagung}

Herrn Prof. Dr. J. Troe möchte ich für die Aufnahme in seine Arbeitsgruppe und die guten Arbeitsbedingungen danken.

Herrn Prof. Dr. B. Abel gilt mein Dank für sein stetes Interesse am Fortschritt der Arbeit, fruchtbare Diskussionen und eine ausgezeichnete Betreuung. Auch seine persönlichen Eigenschaften wie Ausgeglichenheit und Verlässlichkeit haben einen Beitrag zum Gelingen dieser Arbeit geleistet.

Tim Spangenberg danke ich für meine hervorragende Einarbeitung an der Apparatur und die angenehme Zusammenarbeit.

„Meinen‘ Diplomanden Rosalin Karunanandan, Esteban Vöhringer-Martinez und Matthias Ließmann danke ich für die Zusammenarbeit im Labor, die fruchtbaren Diskussionen bei der Bewältigung der technischen Probleme und die Hilfe bei der Ermittlung der zahlreichen Ergebnisse.

Für die Gewährung eines großzügigen Stipendiums möchte ich dem Träger des Graduiertenkollegs 782 „Spektroskopie und Dynamik molekularer Knäuel und Aggregate", der deutschen Forschungsgemeinschaft danken.

Den Mitarbeitern der Werkstätten gilt mein Dank für die Fertigung der Lavaldüsen, die Reparaturen aller möglichen Laborgeräte und die Erstellung von mechanischen und elektronischen Werkstücken aller Art. Dabei hat die praktische Perspektive von Herrn Meyer und Herrn Knorr die optimale Lösung technischer Probleme gewährleistet. Für die Kleinigkeiten des Laborbedarfs und die Beratung bei einigen Bestellungen möchte ich W. Noack alias Milo danken, der zwischen Rohrverschraubungsadapter und CD-Rohlingen immer Zeit für einen kurzen Schnack über Gott und die Welt hatte. 
Ein Danke geht auch an die Kollegen im Schreibraum, Matthias Ließmann und Jaane Seehusen, die fachlichen und fachfremden Diskussionen haben ihren Teil zum Gelingen dieser Arbeit beigetragen.

Mitadministrator Oliver Link danke ich dafür, dass die Arbeitsteilung bezüglich des Computernetzwerks so reibungslos geklappt hat und wir anstehende Probleme oft gemeinsam zum Wohle aller User gemeistert haben. Rainer Oswald gebührt in diesem Zusammenhang Dank für seine kompetente Hilfe, wenn wir mit unserem Latein am Ende waren.

Für das fleißige Korrekturlesen möchte ich mich bei Ravi Fernandez, Matthias Ließmann und Anatoli Maergoiz bedanken, Anatoli ebenso für die Nachhilfe in europäischer und russischer Geschichte in der Mittagspause.

Allen anderen Abteilungsmitgliedern möchte ich für ihre Kollegialität danken.

Sebastian Kühn gilt ein besonderer Dank für seine mittlerweile langjährige Freundschaft und die ausgezeichnete Zusammenarbeit in ungezählten Praktika seit dem ersten Semester. Nach den „Picos de Europa“ werden wir dann wohl auch das Gänseliesel gemeinsam erklimmen.

Hunderte von Kilometern im Sattel mit den „wild strampelnden Männern“ der Radsportabteilung Tuspo Weende haben oft genug dafür gesorgt, die nötige Entspannung vom Doktorandenalltag herbeizuführen um dann mit frischem Elan und Ideen die Arbeit fortzusetzen.

Zuletzt möchte ich meiner Familie danken, die mich stets unterstützt hat. Nelly, es ist schön, dich an meiner Seite zu wissen. 


\section{Lebenslauf}

Am 7. August 1977 wurde ich, Björn Hansmann, als Sohn von Monika Hansmann, geb. Alt, und Ralf Hansmann in Kassel geboren.

Ich besuchte vom September 1984 bis zum Juli 1988 die Grundschule in Söhrewald OT Wellerode. Vom September 1988 bis Juli 1994 besuchte ich die Gesamtschule Söhre in Lohfelden. Danach besuchte ich vom September 1994 bis zum Juni 1997 die Herderschule in Kassel und erwarb die allgemeine Hochschulreife. Meinen Zivildienst leistete ich vom August 1997 bis zum September 1998 im mobilen sozialen Hilfsdienst der Diakoniestation Nord-West der Diakonie in Kassel ab.

Zum Wintersemester 1998/1999 immatrikulierte ich mich an der Georg-AugustUniversität in Göttingen im Studiengang Chemie (Diplom). Dort bestand ich am 26. Oktober 2000 die Diplomvorprüfung. Im Oktober 2002 begann ich meine Diplomarbeit im Arbeitskreis von Prof. Dr. J. Troe mit dem Thema „Die Reaktionen des Hydroxylradikals mit Propen und Isopren bei tiefen Temperaturen", im Juli 2003 erwarb ich das Diplom der Fakultät für Chemie Göttingen.

Vom 1.1.2004 bis zum 31.12.2004 war ich als wissenschaftlicher Mitarbeiter im Sonderforschungsbereich 357 „Molekulare Mechanismen unimolekularer Reaktionen“ beschäftigt. Vom 1.1.2005 bis zum 31.6.2005 erhielt ich ein Promotionsstipendium des Max-Planck-Instituts für Biophysikalische Chemie. Seit dem 1.8.2005 bin ich Stipendiat im Graduiertenkolleg 782 „Spektroskopie und Dynamik molekularer Knäuel und Aggregate“. Als Ergebnis dieser Arbeit entstand die vorliegende Dissertation. 